\title{
AN ANOMALY FORMULA FOR RAY-SINGER METRICS ON MANIFOLDS WITH BOUNDARY
}

\author{
J. BRÜNING AND XIAONAN MA
}

\begin{abstract}
Using the heat kernel, we derive first a local Gauss-BonnetChern theorem for manifolds with a non-product metric near the boundary. Then we establish an anomaly formula for Ray-Singer metrics defined by a Hermitian metric on a flat vector bundle over a Riemannian manifold with boundary, not assuming that the Hermitian metric on the flat vector bundle is flat nor that the Riemannian metric has product structure near the boundary.
\end{abstract}

\section{Introduction}

Let $X$ be a compact $m$-dimensional smooth manifold, not necessarily oriented, with boundary $\partial X=Y$ and canonical embedding $j: Y \rightarrow X$, and let $F$ be a flat complex vector bundle over $X$, equipped with its flat connection $\nabla^{F}$. We denote by $H^{\bullet}(X, F)=\bigoplus_{p=0}^{m} H^{p}(X, F)$ the (absolute) de-Rham cohomology of $X$ with coefficients in $F$. For a finite dimensional vector space $E$, set $\operatorname{det} E:=\Lambda^{\max } E$, and denote by $(\operatorname{det} E)^{-1}:=\operatorname{det} E^{*}$ the dual line. Then it is customary to call the complex line,

$$
\operatorname{det} H^{\bullet}(X, F)=\bigotimes_{p=0}^{m}\left(\operatorname{det} H^{p}(X, F)\right)^{(-1)^{p}},
$$

the determinant of the cohomology of $F$.

Next, choose a Hermitian metric $h^{F}$ on $F$ and a smooth Riemannian metric $g^{T X}$ on $T X$. By Hodge-de Rham theory, the de Rham cohomology $H^{\bullet}(X, F)$ is canonically isomorphic to the kernel of an appropriately chosen Laplacian. Hence the chosen metrics induce a canonical $L^{2}$ - metric $h^{H^{\bullet}(X, F)}$ on $H^{\bullet}(X, F)$. Then the Ray-Singer metric \|\|$_{\operatorname{det} H \bullet(X, F)}^{\mathrm{RS}}$ on $\operatorname{det} H^{\bullet}(X, F)$

Keywords and phrases: Ray-Singer analytic torsion, anomaly formula, characteristic classes

AMS Mathematics Subject Classification: 58J52, 58J28, 58J35 
is defined as the product of the metric induced on $\operatorname{det} H^{\bullet}(X, F)$ by $h^{H^{\bullet}(X, F)}$ with the Ray-Singer analytic torsion [RS], see $\S 4.1$.

If $Y=\emptyset$ and $h^{F}$ is flat, then it is known that \|\|$_{\operatorname{det} H \bullet(X, F)}^{\mathrm{RS}}$ does not depend on $g^{T X}$. Indeed, the celebrated Cheeger-Müller theorem [Ch], [Mü1] tells us that in this case the Ray-Singer metric can be identified with the so called Reidemeister metric which is a topological invariant of the flat bundle $F$ [Mi]. Müller [Mü2] extended his result to the case where $m=\operatorname{dim} X$ is odd and only the metric induced on $\operatorname{det} F$ is required to be flat.

It is natural to ask whether the Ray-Singer metric will be independent of the chosen Hermitian metric on $F$ even without any assumption of flatness. Bismut and Zhang [BiZ1] generalized this discussion to arbitrary flat vector bundles with arbitrary Hermitian metrics and showed that, in even dimensions, the independence ceases to hold. Instead, there is an anomaly formula: the logarithms of two Ray-Singer metrics differ by a local term involving new secondary invariants.

There are also various extensions to the equivariant case, cf. [LR], [Lü], [BiZ2]. Recently, Bismut and Goette [BiG], [Go] obtained a family version of the Bismut-Zhang theorem under the assumption that there exists a fiberwise Morse function for the fibration in question, which generalizes all the above results.

Now consider $X$ with $Y \neq \emptyset$. This case was studied in [LR] and [Lü], $[\mathrm{V}],[\mathrm{H}]$, under the assumption that $h^{F}$ is flat and that $g^{T X}$ has product structure near the boundary. Dai and Fang $[\mathrm{DF}]$ were the first to study this problem with $h^{F}$ flat but without assuming a product structure for $g^{T X}$ near $Y$; they used methods completely different from ours. In [LüS], Lück and Schick computed some examples to show that the boundary term does not vanish in general. We learned about this problem from [DF] which was very helpful for us even though we investigate the problem from a rather different perspective. As an interesting by-product, our reasoning also leads to a local Gauss-Bonnet-Chern theorem [Ch1,2], see Theorem 3.2 below, for manifolds without a product structure near the boundary. As a notable feature of our proof, we derive the boundary contribution from the fundamental solution of the model problem.

Let us now describe the geometric setting in greater detail. Let \|\|$_{\operatorname{det} F}$ be the metric on the line bundle $\operatorname{det} F$ induced by $h^{F}$, and let $g^{T Y}$ be the metric on $T Y$ induced by $g^{T X}$. Denote by $\nabla^{T X}$ and $\nabla^{T Y}$ the Levi-Civita connection on $\left(T X, g^{T X}\right)$ and $\left(T Y, g^{T Y}\right)$, respectively, with curvature $R^{T X}$ and $R^{T Y}$. Let $o(T X)$ be the orientation bundle of $T X$. Let $e\left(T X, \nabla^{T X}\right)$, 
$e\left(T Y, \nabla^{T Y}\right)$ be the associated representatives of the Euler class of $T X, T Y$ in Chern-Weil theory, such that $e\left(T X, \nabla^{T X}\right)$ is an $o(T X)$-valued closed $m$-form on $X$. To account for the boundary, we introduce in (1.17), (1.19) certain forms on $Y, e_{b}\left(Y, \nabla^{T X}\right)$ and $B\left(\nabla^{T X}\right)$, such that $e_{b}\left(Y, \nabla^{T X}\right)$ is an $o(T Y)$-valued $m-1$-form on $Y$. If $m$ is odd, then $e\left(T X, \nabla^{T X}\right)=0$ and $e_{b}\left(Y, \nabla^{T X}\right)=\frac{1}{2} e\left(T Y, \nabla^{T Y}\right)$.

Let $\Omega(X, o(T X)), \Omega(Y, o(T X))$ be the $o(T X)$-valued $C^{\infty}$ forms on $X, Y$. The algebraic mapping cone of $j^{*}: \Omega(X, o(T X)) \rightarrow \Omega(Y, o(T X))$ is defined as the following object: we put $\Omega^{p}(X, Y, o(T X)):=\Omega^{p}(X, o(T X)) \oplus$ $\Omega^{p-1}(Y, o(T X))$ and define, for $\left(\sigma_{1}, \sigma_{2}\right) \in \Omega^{p}(X, Y, o(T X))$, the differential by $d\left(\sigma_{1}, \sigma_{2}\right):=\left(d^{X} \sigma_{1}, j^{*} \sigma_{1}-d^{Y} \sigma_{2}\right)$; then the complex $\left(\Omega^{\bullet}(X, Y, o(T X)), d\right)$ calculates the relative cohomology $H^{\bullet}(X, Y, o(T X))$ [BoT, p. 78]. For $\left(\sigma_{1}, \sigma_{2}\right) \in \Omega(X, Y, o(T X)), \sigma_{3} \in \Omega(X)$, we define a nonsingular pairing

$$
\int_{(X, Y)}\left(\sigma_{1}, \sigma_{2}\right) \wedge \sigma_{3}:=\int_{X} \sigma_{1} \wedge \sigma_{3}-\int_{Y} \sigma_{2} \wedge j^{*} \sigma_{3}
$$

this induces the Poincaré duality $H^{\bullet}(X, Y, o(T X)) \times H^{\bullet}(X, \mathbb{R}) \rightarrow \mathbb{R}$. By Theorem 1.9, the relative differential form

$$
E\left(T X, \nabla^{T X}\right):=\left(e\left(T X, \nabla^{T X}\right), e_{b}\left(Y, \nabla^{T X}\right)\right)
$$

is closed in $\Omega(X, Y, o(T X))$ and defines the relative Euler class of $T X$, and $E(T X):=\left[E\left(T X, \nabla^{T X}\right)\right] \in H^{m}(X, Y, o(T X))$ does not depend on the choice of $g^{T X}$.

Next let $\left(g_{0}^{T X}, h_{0}^{F}\right)$ and $\left(g_{1}^{T X}, h_{1}^{F}\right)$ be two couples of metrics on $(T X, F)$. We will use the subscripts 0,1 to distinguish various objects associated with these metrics. In Theorem 1.9, we show that there exists a canonical class

$$
\widetilde{E}\left(T X, \nabla_{0}^{T X}, \nabla_{1}^{T X}\right) \in \Omega^{m-1}(X, Y, o(T X)) / d \Omega(X, Y, o(T X))
$$

such that

$$
d \widetilde{E}\left(T X, \nabla_{0}^{T X}, \nabla_{1}^{T X}\right)=E\left(T X, \nabla_{1}^{T X}\right)-E\left(T X, \nabla_{0}^{T X}\right) .
$$

It is obvious from (0.4) that $\widetilde{E}$ defines the secondary relative Euler class of $T X$ in the spirit of Chern-Simons theory.

Let $\theta\left(F, h_{1}^{F}\right)=\operatorname{Tr}\left[\left(h_{1}^{F}\right)^{-1} \nabla^{F} h_{1}^{F}\right]$ be the closed 1-form (cf. Definition 4.4), which measures the variation of the metric \|\|$_{\operatorname{det} F, 1}$ on $\operatorname{det} F$ with respect to the induced flat connection on $\operatorname{det} F ; \theta\left(F, h_{1}^{F}\right)$ vanishes if the metric \|\|$_{\operatorname{det} F, 1}$ is flat.

The main result of this paper generalizes [BiZ1, Thm. 0.1] to manifolds with boundary; it reads as follows:

Theorem 0.1. Let $\left(g_{0}^{T X}, h_{0}^{F}\right),\left(g_{1}^{T X}, h_{1}^{F}\right)$ be two couples of metrics on $T X$ and $F$. Then 


$$
\begin{aligned}
& \log \left(\left(\frac{\|\|_{\operatorname{det} H \bullet(X, F), 1}^{\mathrm{RS}}}{\|\|_{\operatorname{det} H \bullet}^{\mathrm{RS}} H^{\prime}(X, F), 0}\right)^{2}\right)=(-1)^{m} \int_{(X, Y)} \log \left(\left(\frac{\|\|_{\operatorname{det} F, 1}}{\|\|_{\operatorname{det} F, 0}}\right)^{2}\right) E\left(T X, \nabla_{0}^{T X}\right) \\
& +\int_{(X, Y)} \widetilde{E}\left(T X, \nabla_{0}^{T X}, \nabla_{1}^{T X}\right) \wedge \theta\left(F, h_{1}^{F}\right)+\operatorname{rk}(F)\left[\int_{Y} B\left(\nabla_{1}^{T X}\right)-\int_{Y} B\left(\nabla_{0}^{T X}\right)\right] .
\end{aligned}
$$

Especially, if $h_{0}^{F}=h_{1}^{F}$ is flat, then (0.5) reads

$$
\log \left(\left(\frac{\|\|_{\operatorname{det} H \bullet(X, F), 1}^{\mathrm{RS}}}{\|\|_{\operatorname{det} H \bullet(X, F), 0}^{\mathrm{RS}}}\right)^{2}\right)=\operatorname{rk}(F)\left[\int_{Y} B\left(\nabla_{1}^{T X}\right)-\int_{Y} B\left(\nabla_{0}^{T X}\right)\right] .
$$

In fact, in [Ch, Cor. 3.29] Cheeger states (what is also implicit in the proof of [RS, Thm. 7.3]) that the left-hand side of (0.6) depends only on the germ of $g_{i}^{T X}$ restricted to $Y$, a crucial fact for Cheeger's proof of the Ray-Singer conjecture [Ch, §4]. In subsection 4.5, we find that our results coincide with the results in [LüS, App. A] where Lück and Schick computed some examples to show that the left-hand side of $(0.6)$ does not vanish in general. Note that $(0.6)$ is different from the corresponding boundary contribution in [DF, Thm.1.1, or $\S 2.2$ and $\S 4.4]$.

Note that in Theorem 0.1, we do not assume that $h_{j}^{F}(j=0,1)$ is flat, nor do we impose any restriction on the metrics $g_{0}^{T X}$ and $g_{1}^{T X}$. To derive the (somewhat mysterious) local contribution from the boundary (i.e. to calculate the asymptotics of the left-hand side in Theorem 4.5), we only do the rescaling of the Clifford variable along $Y$ and, inspired by [BiL, $\S 13 \mathrm{~d}), \mathrm{e})]$, we use a special trivialization of the vector bundles adapted to the boundary geometry, in order to get a manageable limiting boundary value problem. Finally, we introduce two extra Grassmann variables and a strange rescaling.

In this paper, we work out in detail the results associated to the absolute boundary condition on $X$. In [BrüM2], we will give the corresponding results for the relative boundary condition on $X$, and will apply it to derive the gluing formula for the analytic torsion in this general setting. Our results were announced in [BrüM1].

This paper is organized as follows: In section 1, we construct the secondary classes for manifolds with boundary which appear in Theorem 0.1. In section 2, we express the secondary relative Euler class from section 1 in terms of the Mathai-Quillen current. In section 3, we establish a local Gauss-Bonnet-Chern theorem for manifolds with boundary which serves as a model problem for our main result. In section 4, we establish the anomaly formula for the Ray-Singer metric under the additional assumption (4.30), and in section 5 we prove Theorem 0.1 in general. 
Some notation. If the vector space $V=V^{+} \oplus V^{-}$is $\mathbb{Z}_{2}$ graded under the involution $\tau$, and if $A, B \in \operatorname{End}(V)$, then we denote by $[A, B]$ the supercommutator of $A$ and $B$, and put

$$
\operatorname{Tr}_{s}[A]:=\operatorname{Tr}[\tau A] .
$$

We will denote by $i(\cdot)$ and $w(\cdot)$, respectively, the operators induced by the interior and exterior product on forms. For $I=\left\{i_{1}, \ldots, i_{k}\right\} \subset\{1, \ldots, m\}$, and $\left\{e_{i}\right\}$ an orthogonal basis of $V$ with dual basis $\left\{e^{i}\right\}$, we write $w\left(e^{I}\right)=$ $w\left(e^{i_{1}}\right) \circ \cdots \circ w\left(e^{i_{k}}\right)$ and $i\left(e_{I}\right)=i\left(e_{i_{1}}\right) \circ \cdots \circ i\left(e_{i_{k}}\right)$. Indices $\alpha, \beta, \gamma$ will always run from 1 to $m-1$, and indices $i, j, k$ will always run from 1 to $m$.

Acknowledgment. We thank Jean-Michel Bismut for very useful and inspiring conversations. We are indebted to a referee for his very careful reading of the manuscript, and also for very detailed comments. Our collaboration was partially supported by Deutsche Forschungsgemeinschaft through SFB 288 and the European Commission through the Research Training Network "Geometric Analysis". The final revision was done while the second author was visiting Nankai Institute of Mathematics during July and August 2005. He would like to thank Weiping Zhang for hospitality.

\section{Secondary Classes for Manifolds with Boundary}

In this section we define the secondary classes for manifolds with boundary which appear in Theorem 0.1.

This section is organized as follows. In subsection 1.1, we recall the definition of the Berezin integral [BiZ1, §3a)]. In subsection 1.2, we describe the geometric setting, especially the connection from [Bi1, Def. 1.6]. In subsection 1.3, we state a technical result which we prove in subsection 1.4. In subsection 1.5, we construct the secondary classes for a fibration of manifolds with boundary.

1.1 The Berezin integral. For $\mathbb{Z}_{2}$-graded algebras $\mathcal{A}, \mathcal{B}$ with identity, we introduce the $\mathbb{Z}_{2}$-graded tensor product $\mathcal{A} \widehat{\otimes} \mathcal{B}$ and define $\mathcal{A}:=\mathcal{A} \widehat{\otimes} I$, and $\widehat{\mathcal{B}}:=I \widehat{\otimes} \mathcal{B}$, and we write $\wedge:=\widehat{\otimes}$ such that $\mathcal{A} \widehat{\otimes} \mathcal{B}=\mathcal{A} \wedge \widehat{\mathcal{B}}$. Let $E$ and $V$ be finite dimensional real vector spaces of dimension $n$ and $l$, respectively. Assume that $E$ is Euclidean and oriented, with oriented orthonormal basis $\left\{e_{i}\right\}_{i=1}^{n}$ and dual basis $\left\{e^{i}\right\}_{i=1}^{n}$ with respect to the Euclidean metric $h^{E}$, and denote by $\Lambda E^{*}$ the exterior algebra of $E^{*}$. Then the Berezin integral we use is the linear map

$$
\int^{B}: \Lambda V^{*} \wedge \widehat{\Lambda E^{*}} \rightarrow \Lambda V^{*}, \quad \alpha \wedge \hat{\beta} \mapsto c_{B} \beta\left(e_{1}, \ldots, e_{n}\right) \alpha,
$$


where the normalizing constant is given by $c_{B}:=(-1)^{n(n+1) / 2} \pi^{-n / 2}$. More generally, for any Euclidean space $E$ with orientation line $o(E)$, the Berezin integral maps $\Lambda V^{*} \wedge \widehat{\Lambda E^{*}}$ into $\Lambda V^{*} \otimes o(E)$.

In the sequel, we no longer assume that $E$ is oriented. Let $C$ be an antisymmetric endomorphism of $E$. We identify $C$ with the element of $\widehat{\Lambda E^{*}}$ given by

$$
\dot{C}=\frac{1}{2} \sum_{1 \leq i, j \leq n}\left\langle e_{i}, C e_{j}\right\rangle \widehat{e^{i}} \wedge \widehat{e^{j}} .
$$

This being understood, the Pfaffian $\operatorname{Pf}[C / 2 \pi]$ of $C / 2 \pi$ is defined by

$$
\int^{B} \exp \left(\frac{-\dot{C}}{2}\right)=\operatorname{Pf}\left[\frac{C}{2 \pi}\right] \in o(E) \text {. }
$$

Clearly, $\operatorname{Pf}[C / 2 \pi]$ vanishes if $n$ is odd.

1.2 The canonical connection of a fibration. Let $W$ be a smooth manifold with boundary $V$. Let $S$ be another manifold and let $\pi_{W}: W \rightarrow S$ be a fibration with $m$-dimensional compact fiber $X$, which induces a fibration $\pi_{V}: V \rightarrow S$ with compact fiber $Y$ such that $Y=\partial X$. Let $T X, T Y$ be the corresponding tangent bundles along the fibers, and let $T^{H} W \subset T W$ be a horizontal subbundle such that $T^{H} W \oplus T X=T W$, with projections $P^{T^{H} W}$ and $P^{T X}$. For $U \in T S$, we denote by $U^{H} \in T^{H} W$ its horizontal lift.

Let $g^{T X}$ be a metric on $T X$ and denote by $g^{T Y}$ the metric on $T Y$ induced by $g^{T X}$. Let $N$ be the (orientable) normal bundle to $Y$ in $X$ which we identify with the orthogonal complement of $T Y$ in $T X$.

Definition 1.1. The canonical metric connection $\nabla^{T X}$ on $\left(T X \rightarrow W, g^{T X}\right)$ is defined by the following properties:

a) On each fiber $X, \nabla^{T X}$ restricts to the Levi-Civita connection of $\left(T X, g^{T X}\right)$.

b) If $U \in T S$, then

$$
\nabla_{U^{H}}^{T X}=\mathcal{L}_{U^{H}}+\frac{1}{2}\left(g^{T X}\right)^{-1}\left(\mathcal{L}_{U^{H}} g^{T X}\right) .
$$

We recall a simple result stated in [BiGS1, Thm. 1.2] (cf. [Bi3, Thms. 1.1 and 1.2]) the proof of which can also be found in [BGV, Prop. 10.2].

Theorem 1.2. If $g^{T W}$ is a metric on $T W$ with Levi-Civita connection $\nabla^{T W}$ such that $g^{T W}$ induces the given metric $g^{T X}$ on $T X$, and $T^{H} W$ is the orthogonal bundle to $T X$ in $T W$ with respect to $g^{T W}$, then

$$
\nabla^{T X}=P^{T X} \nabla^{T W} \text {. }
$$

On $V$, we denote by $P^{T Y}$ and $P^{N}$ the orthogonal projections from $\left.T X\right|_{V}$ onto $T Y$ and $N$ respectively. Set $\nabla^{T Y}:=P^{T Y} \nabla^{T X \mid V}$ and let $R^{T Y}$ be the curvature of $\nabla^{T Y}$. 
Theorem 1.3 [Bi3, Thm. 1.9]. Set $T^{H} V:=\left(T^{H} W \oplus N\right) \cap T V$. Then $\nabla^{T Y}$ is the canonical connection on $T Y$ defined by the data $\left(\pi_{V}, T^{H} V, g^{T Y}\right)$.

Proof. Let $g^{T V}$ be the metric on $T V$ induced by $g^{T W}$ in Theorem 1.2; then $T^{H} V$ is the orthogonal bundle to $T Y$ in $T V$ with respect to $g^{T V}$. Also on $V, T W=T^{H} V \oplus T Y \oplus N$. If $P^{T V}$ is the orthogonal projection $\left.T W\right|_{V} \rightarrow T V$ and if $\nabla^{T V}$ is the Levi-Civita connection on $\left(T V, g^{T V}\right)$, then clearly $\nabla^{T V}=P^{T V} \nabla^{T W}$. Thus

$$
\nabla^{T Y}=P^{T Y} P^{T X} \nabla^{T W}=P^{T Y} P^{T V} \nabla^{T W}=P^{T Y} \nabla^{T V},
$$

and Theorem 1.3 follows from (1.5).

For later use, it is useful to extend the splitting $\left.T X\right|_{V}=T Y \oplus N$ to a neighborhood of $V$. We suppose that $W$ is compact. Let $e_{\mathfrak{n}}$ be the inward pointing unit normal at any boundary point. Then there exists $\varepsilon>0$ such that for any $y \in V$, $\exp _{y}^{X}\left(u e_{\mathfrak{n}}(y)\right) \in W$, the geodesic in the fiber $X$ with respect to $g^{T X}$, is well defined for $u \in[0, \varepsilon)$; we use this map to identify $V \times[0, \varepsilon)$ with $\mathcal{U}_{\varepsilon}$, a neighborhood of $V$ in $W$; we denote by $\pi_{\varepsilon}: V \times[0, \varepsilon) \rightarrow V$ the natural projection. Then there exists a family of metrics, $g^{T Y}\left(x_{m}\right)$, on $T Y$ such that

$$
g^{T X}\left(y, x_{m}\right)=d x_{m}^{2}+g^{T Y}\left(x_{m}\right), \quad\left(y, x_{m}\right) \in V \times[0, \varepsilon) .
$$

Let ${ }^{1} T X=\pi_{\varepsilon}^{*} T Y,{ }^{2} T X=\pi_{\varepsilon}^{*} N$, then ${ }^{1} T X$ and ${ }^{2} T X$ are smooth orthogonal sub-bundles of $\left.T X\right|_{\varepsilon}$, and ${ }^{2} T X$ is the trivial line bundle with the frame $e_{m}=\partial / \partial x_{m}$; and for $x=\left(y, x_{m}\right) \in \mathcal{U}_{\varepsilon},{ }^{1} T X_{x},{ }^{2} T X_{x}$ are obtained by parallel transport of $T_{y} Y, N_{y}$ with respect to the connection $\nabla^{T X}$ along the geodesic $[0,1] \ni u \mapsto\left(y, u x_{m}\right)$. For $j=1,2$, let $P^{j} T X$ be the orthogonal projection operator from $\left.T X\right|_{\mathcal{U}_{\varepsilon}}$ onto ${ }^{j} T X$, and denote by $\nabla^{j} T X$ the connection on ${ }^{j} T X$ given by $\nabla^{j} T X=P^{j} T X \nabla^{T X}$. Then the restriction of $\nabla^{1} T X$ to $V$ coincides with $\nabla^{T Y}$. Next let ${ }^{s p} \nabla^{T X}=\nabla^{1 T X} \oplus \nabla^{2} T X$ be the direct sum connection on $T X={ }^{1} T X \oplus^{2} T X$ with curvature ${ }^{s p} R^{T X}$ (where "sp" refers to "split"), and set

$$
A:=\nabla^{T X}-{ }^{s p} \nabla^{T X}=P^{1} T X \nabla^{T X} P^{2} T X+P^{2} T X \nabla^{T X} P^{1} T X .
$$

Then $A$ is a 1 -form on $\mathcal{U}_{\varepsilon_{0}}$ taking values in the skew-adjoint endomorphisms of $T X$ which exchange ${ }^{1} T X$ and ${ }^{2} T X$. By construction,

$$
\begin{array}{cc}
{ }^{s p} \nabla^{T X} e_{m}=0, & \nabla_{e_{m}}^{T X} e_{m}=0 \quad \text { on } \quad \mathcal{U}_{\varepsilon}, \\
A\left(e_{m}\right)=0, & \left.{ }^{s p} R^{T X}\right|_{V}=R^{T Y} \quad \text { on } \quad V .
\end{array}
$$

Moreover, $A$ defines the second fundamental form of $Y$ which measures the deviation from a metric product structure near the boundary: if $y_{0} \in V$ and $Z_{1}, Z_{2} \in T_{y_{0}} Y$, we choose extensions $\widetilde{Z}_{1}, \widetilde{Z}_{2}$ to vector fields on $\mathcal{U}_{\varepsilon}$ by 
extending first to $Y$ and then trivially to $V \times[0, \varepsilon)$; then we get at $y_{0}$ $\left\langle A\left(Z_{1}\right) Z_{2}, e_{m}\right\rangle=\left\langle\nabla_{Z_{1}}^{T X} \widetilde{Z}_{2}, e_{m}\right\rangle=-\left\langle\nabla_{e_{m}}^{T X} \widetilde{Z}_{1}, Z_{2}\right\rangle=-\frac{1}{2}\left(\mathcal{L}_{e_{m}} g^{T X}\right)\left(Z_{1}, Z_{2}\right)$.

In particular, $A_{y_{0}}\left(Z_{1}\right) Z_{2}$ is symmetric for $Z_{1}, Z_{2} \in T_{y_{0}} Y$. From (1.8), and (1.9), we get the Gauss-Codazzi equation for the second fundamental form (cf. [C, §6.3]): If $y_{0} \in V, Z_{1}, Z_{2} \in T_{y_{0}} Y, W_{1}, W_{2} \in T_{y_{0}} X$, then

$$
\begin{array}{r}
\left\langle\left(R_{y_{0}}^{T X}-{ }^{s p} R_{y_{0}}^{T X}\right)\left(W_{1}, W_{2}\right) Z_{1}, Z_{2}\right\rangle_{g^{T X}}=\left\langle A_{y_{0}}^{2}\left(W_{1}, W_{2}\right) Z_{1}, Z_{2}\right\rangle_{g^{T X}} \\
=\left\langle A_{y_{0}}^{2}\left(P^{T Y} W_{1}, P^{T Y} W_{2}\right) Z_{1}, Z_{2}\right\rangle_{g^{T X}} .
\end{array}
$$

For $U \in T S$, let $U^{H, V} \in T^{H} V \subset T V$ be the lift of $U$, and $U_{\left(y, x_{m}\right)}^{H, N}=$ $U_{\left(y, x_{m}\right)}^{H}-U_{y}^{H, V} \in T X$ for $\left(y, x_{m}\right) \in V \times[0, \varepsilon)$, then $U^{H, N} \in N$ on $V$. Moreover, $\left[U^{H, V}, e_{m}\right]=0$, thus by $(1.7),\left(\mathcal{L}_{U^{H, V}} g^{T X}\right) e_{m}=0$. By (1.4), (1.7) and (1.9), on $V$

$A\left(U^{H, V}\right) e_{m}=\nabla_{U^{H}-U^{H, N}}^{T X} e_{m}=\nabla_{U^{H}}^{T X} e_{m}=\mathcal{L}_{U^{H, N}} e_{m}+\frac{1}{2}\left(g^{T X}\right)^{-1}\left(\mathcal{L}_{U^{H, N}} g^{T X}\right) e_{m}$.

1.3 An identity for $\boldsymbol{e}_{\boldsymbol{b}}$. If $\boldsymbol{\omega}$ is a smooth section of $\Lambda\left(T^{*} W\right)$ we identify $\omega$ with the section $\omega \widehat{\otimes} 1$ of $\left.\Lambda\left(T^{*} W\right) \widehat{\otimes} \Lambda \widehat{\left(T^{*} W\right.}\right) ; \widehat{\omega}$ will denote the section $1 \widehat{\otimes} \widehat{\omega}$ of $\Lambda\left(T^{*} W\right) \widehat{\otimes} \Lambda\left(\widehat{T^{*} W}\right)$ as before. We will apply the Berezin integral from subsection 1.1 to $\left.\Lambda\left(T^{*} W\right) \widehat{\otimes} \widehat{\Lambda\left(T^{*} X\right.}\right)$ and $\left.\Lambda\left(T^{*} V\right) \widehat{\otimes} \widehat{\Lambda\left(T^{*} Y\right.}\right)$, and, for convenience, we will denote this operation by $\int^{B_{X}}$ and $\int^{B_{Y}}$, respectively.

Let $\left\{e_{i}\right\}_{i=1}^{m}$ be an orthonormal frame of $\left(T X, g^{T X}\right)$ and let $\left\{e^{i}\right\}$ be the corresponding dual frame of $T^{*} X$. Set

$$
\left.\dot{R}^{T X}:=\frac{1}{2} \sum_{1 \leq i, j \leq m}\left\langle e_{i}, R^{T X} e_{j}\right\rangle \widehat{e^{i}} \wedge \widehat{e^{j}} \in \Lambda^{2}\left(T^{*} W\right) \widehat{\otimes} \Lambda^{2\left(T^{*} X\right.}\right) .
$$

Let $j: V \hookrightarrow W$ be the natural embedding. We only consider orthonormal frames $\left\{e_{i}\right\}_{i=1}^{m}$ of $T X$ with the property that near the boundary $V, e_{m}=: e_{\mathfrak{n}}$ is the inward-pointing unit normal at any boundary point. We will use Greek indices to specify the induced frame of $T Y$, such that $\left\{e_{\alpha}\right\}_{\alpha=1}^{m-1}$ denotes a local orthonormal frame for $T Y$.

Let $o(T X)$ be the orientation bundle of $T X$, which is a flat real line bundle on $X$ [BoT, p. 88]. To compare the Berezin integrals $\int^{B_{X}}$ and $\int^{B_{Y}}$, we identify $\left.o(T X)\right|_{Y}$ with $o(T Y)$ as follows: as explained in [BoT, p. 88], we can view $o(T X)$ as the real line bundle $\operatorname{det}\left(T^{*} X\right)$ on the fiber $X$, with transition functions in $\{ \pm 1\}$; then we identify the section $\left.\left(-e^{m}\right) \wedge \sigma \in \operatorname{det}\left(T^{*} X\right)\right|_{Y}$ with $\sigma \in \operatorname{det}\left(T^{*} Y\right)$ on $Y$. Consequently, for $\gamma \in \Lambda\left(T^{*} W\right)$ and $\left.\beta \in \widehat{\Lambda\left(T^{*} Y\right.}\right)$ 
on $Y$, we have

$$
\int^{B_{X}} \gamma \beta \wedge \widehat{e^{m}}=\pi^{-1 / 2} \int^{B_{Y}} \gamma \beta .
$$

(Assume $W=X$. If $X$ is orientable and $\left(-e^{m}\right) \wedge e^{1} \wedge \cdots \wedge e^{m-1}$ defines the orientation of $X$, then (1.14) means that $e^{1} \wedge \cdots \wedge e^{m-1}$ is the induced orientation on $Y$, such that the Stokes theorem, $\int_{X} d \gamma=\int_{Y} \gamma$, holds for $\gamma \in \Omega(X)$. In general, if $\left.\gamma \in \Omega\left(X, \widehat{\Lambda^{m}\left(T^{*} X\right.}\right)\right)$ such that $\gamma=\gamma_{1} \wedge \widehat{e^{m}}$ on $Y$, then (1.14) means that $\int_{X} d \int^{B_{X}} \gamma=\pi^{-1 / 2} \int_{Y} \int^{B_{Y}} \gamma_{1}$; this equation holds even if $X$ is not orientable.)

On $V$, set

$$
\begin{aligned}
& \left.\dot{S}:=\frac{1}{2} j^{*} \nabla^{T X} \widehat{e^{m}}=-\frac{1}{2} \sum_{\beta=1}^{m-1}\left\langle e_{\mathfrak{n}}, A e_{\beta}\right\rangle \widehat{e^{\beta}} \quad \in T^{*} V \widehat{\otimes} \widehat{\Lambda^{1}\left(T^{*} Y\right.}\right), \\
& j^{*} \dot{R}^{T X}:=\frac{1}{2} \sum_{1 \leq i, j \leq m}\left\langle e_{i}, j^{*} R^{T X} e_{j}\right\rangle \widehat{e^{i}} \wedge \widehat{e^{j}} \quad \in \Lambda^{2}\left(T^{*} V\right) \widehat{\otimes} \widehat{\Lambda^{2}\left(T^{*} X\right)}, \\
& \left.\dot{R}^{T X} \mid Y:=\frac{1}{2} \sum_{1 \leq \gamma, \delta \leq m-1}\left\langle e_{\gamma}, j^{*} R^{T X} e_{\delta}\right\rangle \widehat{e^{\gamma}} \wedge \widehat{e^{\delta}} \quad \in \Lambda^{2}\left(T^{*} V\right) \widehat{\otimes} \Lambda^{2\left(T^{*} Y\right.}\right), \\
& \left.\dot{R}^{T Y}:=\frac{1}{2} \sum_{1 \leq \gamma, \delta \leq m-1}\left\langle e_{\gamma}, R^{T Y} e_{\delta}\right\rangle \widehat{e^{\gamma}} \wedge \widehat{e^{\delta}} \in \Lambda^{2}\left(T^{*} V\right) \widehat{\otimes} \widehat{\Lambda^{2}\left(T^{*} Y\right.}\right) .
\end{aligned}
$$

By (1.8) and (1.11), we have

$$
\dot{R}^{T Y}=\dot{R}^{T X} \mid Y-2 \dot{S}^{2}
$$

The Chern-Weil forms $e\left(T X, \nabla^{T X}\right):=\operatorname{Pf}\left[R^{T X} / 2 \pi\right]$ and $e\left(T Y, \nabla^{T Y}\right):=$ $\operatorname{Pf}\left[R^{T Y} / 2 \pi\right]$ are closed and $e\left(T X, \nabla^{T X}\right)$ is an $o(T X)$-valued $m$-form on $W$ which represents the Euler class of $T X$. On $V$, we further introduce

$$
\begin{aligned}
& e_{b}\left(V, \nabla^{T X}\right):=(-1)^{m-1} \int^{B_{Y}} \exp \left(-\frac{1}{2}\left(\dot{R}^{T X} \mid Y\right)\right) \sum_{k=0}^{\infty} \frac{\dot{S}^{k}}{2 \Gamma\left(\frac{k}{2}+1\right)}, \\
& \mu\left(\nabla^{T X}\right):=\sqrt{\pi} \int^{B_{X}} \exp \left(-\frac{1}{2} j^{*} \dot{R}^{T X}\right) \sum_{k=1}^{\infty} \frac{\dot{S}^{k-1}}{2 \Gamma\left(\frac{k}{2}+1\right)}, \\
& B\left(\nabla^{T X}\right):=-\int_{0}^{1} \frac{d u}{u} \int^{B_{Y}} \exp \left(-\frac{1}{2} \dot{R}^{T Y}-u^{2} \dot{S}^{2}\right) \sum_{k=1}^{\infty} \frac{(u \dot{S})^{k}}{2 \Gamma\left(\frac{k}{2}+1\right)} .
\end{aligned}
$$

(There is a misprint in the definition of $B\left(\nabla^{T X}\right)$ in [BrüM1, (14)], the right-hand side must be multiplied by -1.) Then $e_{b}\left(V, \nabla^{T X}\right), B\left(\nabla^{T X}\right)$ (resp. $\left.\mu\left(\nabla^{T X}\right)\right)$ are $m-1$ (resp. $m$ )-forms on $V$ with values in $o(T Y)$. If 
$\operatorname{dim} X=m$ is odd, then by (1.16) and (1.17),

$$
\begin{aligned}
e_{b}\left(V, \nabla^{T X}\right) & =\int^{B_{Y}} \exp \left(-\frac{1}{2}\left(\dot{R}^{T X} \mid Y\right)\right) \sum_{k=0}^{\infty} \frac{\dot{S}^{2 k}}{2 \Gamma(k+1)} \\
& =\frac{1}{2} \int^{B_{Y}} \exp \left(-\frac{1}{2} \dot{R}^{T Y}\right)=\frac{1}{2} e\left(T Y, \nabla^{T Y}\right), \\
B\left(\nabla^{T X}\right) & =\int_{0}^{1} \int^{B_{Y}} \exp \left(-\frac{1}{2} \dot{R}^{T Y}\right)\left(\exp \left(-u^{2} \dot{S}^{2}\right)-1\right) \frac{d u}{2 u} \\
& =\int^{B_{Y}} \exp \left(-\frac{1}{2} \dot{R}^{T Y}\right) \sum_{k=1}^{\infty} \frac{\left(-\dot{S}^{2}\right)^{k}}{4 k \Gamma(k+1)} .
\end{aligned}
$$

Now we present the main result of this subsection; its proof will be given in the next subsection.

Theorem 1.4. On $V$ we have

$$
\begin{gathered}
d^{V} e_{b}\left(V, \nabla^{T X}\right)=j^{*} e\left(T X, \nabla^{T X}\right), \\
d^{V} B\left(\nabla^{T X}\right)=\mu\left(\nabla^{T X}\right) .
\end{gathered}
$$

REMARK 1.5. If $\operatorname{dim} X=m$ is even, then by (1.32), (1.33) and (1.34), $e_{b}\left(V, \nabla^{T X}\right)$ represents the Chern-Simons class $\widetilde{e}\left(V, \nabla^{T X},{ }^{s p} \nabla^{T X}\right)$.

With the notation of subsection 1.4 (cf. (1.36), (1.40)), we get in general

$$
B\left(\nabla^{T X}\right)=(-1)^{m} \int_{0}^{1} \frac{d s}{s}\left[e_{b}\left(V, \nabla_{s}^{T X}\right)-\frac{1}{2} \int^{B_{Y}} \exp \left(-\frac{1}{2}\left(\dot{R}_{s}^{T X} \mid Y\right)\right)\right] .
$$

1.4 Proof of Theorem 1.4. Set $\widetilde{W}=W \times \mathbb{R}, \widetilde{j}:=j \times \operatorname{id}_{\mathbb{R}}, \pi_{\widetilde{W}}:=$ $\pi_{W} \times \operatorname{id}_{\mathbb{R}}: \widetilde{W} \rightarrow S \times \mathbb{R}$. Let $p_{W}: \widetilde{W} \rightarrow W, p_{V}: \widetilde{V}:=V \times \mathbb{R} \rightarrow V$, $p_{\mathbb{R}}: \widetilde{W} \rightarrow \mathbb{R}$ be the natural projections. We denote by $\widetilde{X}$ the fibers with boundary $\widetilde{Y}$ of the fibration $\pi_{\widetilde{W}}$. We will denote by $T \widetilde{X}$ the tangent bundle along the fiber of $\pi_{\widetilde{W}}$.

We only need to prove Theorem 1.4 on compact subsets of $S$, thus we may assume $S$ compact. We use the notation in subsections 1.2, 1.3. Let $g_{0}^{T X}$ be a smooth metric on $T X$ such that on $V \times[0, \varepsilon)$,

$$
g_{0}^{T X}\left(y, x_{m}\right)=d x_{m}^{2}+g^{T Y}(0) .
$$

Let $g^{T \widetilde{X}}$ be a smooth metric on the bundle $T \widetilde{X}$ over $\widetilde{W}$ such that for $(x, s) \in W \times[0,1]$, with $g_{1}^{T X}:=g^{T X}$,

$$
g^{T \widetilde{X}}(x, s)=(1-s) g_{0}^{T X}+s g_{1}^{T X} .
$$

We extend $T^{H} W$ on $W \times\{0\}$ to $\widetilde{T}^{H} W$ on $\widetilde{W}$ such that on $V \times[0, \varepsilon) \times \mathbb{R}$, for $U \in T S, y \in V, x_{m} \in[0, \varepsilon), s \in[0,1]$,

$$
U^{H}\left(y, x_{m}, s\right)=(1-s) U^{H, V}(y)+s U^{H}\left(y, x_{m}\right) .
$$

Set $T^{H} \widetilde{W}=\widetilde{T}^{H} W \oplus p_{\mathbb{R}}^{*} T \mathbb{R}$. 
Now we apply the construction in subsection 1.2 to this new fibration $\left(\pi_{\widetilde{W}}, T^{H} \widetilde{W}, g^{T \widetilde{X}}\right)$. Thus let $\nabla^{T \widetilde{X}}$ be the canonical connection on $T \widetilde{X}$ with curvature $R^{T \tilde{X}}$, and let $\widetilde{A}, \dot{\widetilde{S}}$ be the tensors on $V \times \mathbb{R}=\partial W \times \mathbb{R}$ defined by (1.8) and (1.15). Let $\nabla_{s}^{T X}$ be the connection on $T X$ induced by the restriction of $\nabla^{T \tilde{X}}$ on $W \times\{s\}$ and denote its curvature by $R_{s}^{T X}$. By (1.4) and (1.23), $\nabla_{\partial / \partial s}^{T \widetilde{X}} e_{\mathfrak{n}}=0$ on $\partial W \times[0,1]$. Thus for $(y, s) \in \partial W \times \mathbb{R}$ and $s \in[0,1]$, we get from (1.10), (1.12), (1.23) and (1.24),

$$
\widetilde{A}(y, s)=s A(y), \quad \dot{\widetilde{S}}(y, s)=s \dot{S}(y) .
$$

Proposition 1.6. If $\operatorname{dim} X$ is odd, then on $V \times \mathbb{R}$,

$$
d^{V \times \mathbb{R}} \int^{B_{X}} \widehat{e^{m}} \wedge \exp \left(-\frac{1}{2} \widetilde{j}^{*} \dot{R}^{T \widetilde{X}}\right)=\int^{B_{X}} 2 \dot{\widetilde{S}} \wedge \exp \left(-\frac{1}{2} \widetilde{j}^{*} \dot{R}^{T \widetilde{X}}\right) .
$$

Proof. The Bianchi identity asserts that $\left[\nabla^{T \widetilde{X}}, \dot{R}^{T \widetilde{X}}\right]=0$ on $\widetilde{W}$. As the connection $\nabla^{T \widetilde{X}}$ preserves the metric $g^{T \widetilde{X}}$, we have, for any smooth section $\sigma$ of $\Lambda\left(T^{*} \widetilde{W}\right) \widehat{\otimes} \Lambda\left(T^{*} \widetilde{X}\right), d^{\widetilde{W}} \int^{B_{X}} \sigma=\int^{B_{X}} \nabla^{T \widetilde{X}} \sigma$ (cf. [BGV, Prop. 1.50]). Thus with (1.15) we get (1.26).

Lemma 1.7. For $s \in[0,1]$,

$$
\begin{aligned}
\left.\widetilde{j}^{*} \dot{R}^{T \widetilde{X}}\right|_{V \times\{s\}}= & \dot{R}^{T X} \mid Y-2\left(1-s^{2}\right) \dot{S}^{2} \\
& +s \sum_{\alpha=1}^{m-1}\left\langle e_{\alpha}, j^{*} R^{T X} e_{m}\right\rangle \widehat{e^{\alpha}} \wedge \widehat{e^{m}}+2 d s \wedge \dot{S} \wedge \widehat{e^{m}} .
\end{aligned}
$$

Proof. From (1.8), with our notation for supercommutators,

$$
j^{*} R^{T X}={ }^{s p} R^{T X}+\left[{ }^{s p} \nabla^{T X \mid V}, A\right]+A^{2} .
$$

By (1.8), (1.23), and (1.25), we get, on $\partial W \times[0,1]$,

$$
\widetilde{j}^{*} \nabla^{T \widetilde{X}}={ }^{s p} \nabla^{T \widetilde{X} \mid V \times \mathbb{R}}+s p_{V}^{*} A, \quad{ }^{s p} \nabla^{T \widetilde{X} \mid V \times \mathbb{R}}=p_{V}^{*}{ }^{s p} \nabla^{T X \mid V}+d s \wedge \mathcal{L}_{\partial / \partial s} .
$$

Thus

$$
\left.\widetilde{j}^{*} R^{T \widetilde{X}}\right|_{V \times\{s\}}={ }^{s p} R^{T X}+s^{2} p_{V}^{*} A^{2}+d s \wedge p_{V}^{*} A+s p_{V}^{*}\left[{ }^{s p} \nabla^{T X \mid V}, A\right] .
$$

From (1.15), (1.16), (1.28), and (1.30), we get (1.27).

Proof of Theorem 1.4. Note that for $k \in \mathbb{N}$,

$$
\int_{0}^{1}\left(1-s^{2}\right)^{k} d s=\frac{1}{2} \int_{-1}^{1}\left(1-s^{2}\right)^{k} d s=2^{2 k} \int_{0}^{1} s^{k}(1-s)^{k} d s=\frac{2^{2 k}(k !)^{2}}{(2 k+1) !} .
$$

Let $\delta_{1, s}, \mu_{1, s} \in C^{\infty}\left(W, \Lambda\left(T^{*} W\right) \otimes o(T X)\right), \delta_{2, s}, \mu_{2, s} \in C^{\infty}\left(V, \Lambda\left(T^{*} V\right) \otimes o(T X)\right)$ be forms such that $\left.e\left(T \widetilde{X}, \nabla^{T \widetilde{X}}\right)\right|_{W \times\{s\}}=\left.\int^{B_{X}} \exp \left(-\frac{1}{2} \dot{R}^{T \tilde{X}}\right)\right|_{W \times\{s\}}=p_{W}^{*} \mu_{1, s}+d s \wedge p_{W}^{*} \delta_{1, s}$, 


$$
\left.\int^{B_{X}} \dot{\widetilde{S}} \exp \left(-\frac{1}{2} \widetilde{j}^{*} \dot{R}^{T \widetilde{X}}\right)\right|_{V \times\{s\}}=p_{V}^{*} \mu_{2, s}+d s \wedge p_{V}^{*} \delta_{2, s}
$$

As $e\left(T \widetilde{X}, \nabla^{T \widetilde{X}}\right)$ is closed on $\widetilde{W},(1.32)$ implies

$$
d^{W} \delta_{1, s}=\frac{\partial}{\partial s} \int^{B_{X}} \exp \left(-\frac{1}{2} \dot{R}_{s}^{T X}\right) .
$$

If $\operatorname{dim} X$ is even, by (1.14), (1.27), and (1.31), we have on $V$

$$
\begin{aligned}
& \int_{0}^{1} d s j^{*} \delta_{1, s}=\int_{0}^{1} d s \int^{B_{X}} \exp \left(-\frac{1}{2} \dot{R}^{T X} \mid Y+\left(1-s^{2}\right) \dot{S}^{2}\right)\left(-\dot{S} \wedge \widehat{e^{m}}\right) \\
= & -\frac{1}{\sqrt{\pi}} \int^{B_{Y}} \exp \left(-\frac{1}{2} \dot{R}^{T X} \mid Y\right) \sum_{k=0}^{\infty} \frac{2^{2 k} k !}{(2 k+1) !} \dot{S}^{2 k+1}=e_{b}\left(V, \nabla^{T X}\right) .
\end{aligned}
$$

From (1.33) and (1.34), we get (1.20a).

Applying (1.20a) on $W \times \mathbb{R}$, we get $d^{\widetilde{V}} e_{b}\left(\widetilde{V}, \nabla^{T \widetilde{X}}\right)=\widetilde{j}^{*} e\left(T \widetilde{X}, \nabla^{T \widetilde{X}}\right)$, and by (1.17), the component in $\Lambda\left(T^{*} V\right) \otimes o(T X)$ of $e_{b}\left(\widetilde{V}, \nabla^{T \widetilde{X}}\right)$ is $e_{b}\left(V, \nabla_{s}^{T X}\right)$. Thus (1.32) implies

$$
j^{*} \mu_{1, s}=d^{V} e_{b}\left(V, \nabla_{s}^{T X}\right) .
$$

Thus by (1.16), (1.17), (1.25), (1.27), and (1.35),

$$
\int_{0}^{1} \frac{d s}{s} j^{*} \mu_{1, s}=d^{V} \int_{0}^{1} \frac{d s}{s} e_{b}\left(V, \nabla_{s}^{T X}\right)=d^{V} B\left(\nabla^{T X}\right) .
$$

But by (1.17), (1.27), (1.31) and (1.32), we have

$$
\begin{gathered}
\int_{0}^{1} \frac{d s}{s} j^{*} \mu_{1, s}=\int_{0}^{1} d s \int^{B_{X}} \exp \left(-\frac{1}{2} \dot{R}^{T X} \mid Y+\left(1-s^{2}\right) \dot{S}^{2}\right) \\
\times\left(-\frac{1}{2}\right) \sum_{\alpha=1}^{m-1}\left\langle e_{\alpha}, j^{*} R^{T X} e_{m}\right\rangle \widehat{e^{\alpha}} \wedge \widehat{e^{m}} \\
=\int_{0}^{1} d s \int^{B_{X}} \exp \left(-\frac{1}{2} j^{*} \dot{R}^{T X}+\left(1-s^{2}\right) \dot{S}^{2}\right) \\
=\sqrt{\pi} \int^{B_{X}} \exp \left(-\frac{1}{2} j^{*} \dot{R}^{T X}\right) \sum_{k=0}^{\infty} \frac{\dot{S}^{2 k}}{2 \Gamma\left(k+\frac{3}{2}\right)}=\mu\left(\nabla^{T X}\right) .
\end{gathered}
$$

This gives (1.20b) in the even dimensional case.

If $\operatorname{dim} X$ is odd, then $e_{b}\left(V, \nabla^{T X}\right)$ is closed on $V$ by (1.18). From (1.17), $(1.25),(1.27)$ and (1.32), as in (1.37) 


$$
\begin{gathered}
\sqrt{\pi} \int_{0}^{1} \frac{d s}{s} \mu_{2, s}=\sqrt{\pi} \int_{0}^{1} d s \int^{B_{X}} s \dot{S} \exp \left(-\frac{1}{2} \dot{R}^{T X} \mid Y+\left(1-s^{2}\right) \dot{S}^{2}\right) \\
\times \frac{-1}{2} \sum_{\alpha=1}^{m-1}\left\langle e_{\alpha}, j^{*} R^{T X} e_{m}\right\rangle \widehat{e^{\alpha}} \wedge \widehat{e^{m}} \\
=\sqrt{\pi} \int_{0}^{1} d s \int^{B_{X}} \exp \left(-\frac{1}{2} j^{*} \dot{R}^{T X}+\left(1-s^{2}\right) \dot{S}^{2}\right) s \dot{S}=\mu\left(\nabla^{T X}\right) .
\end{gathered}
$$

By (1.16), (1.25) and (1.27), at $s \in[0,1]$,

$$
\dot{R}^{T \widetilde{X}}\left|\widetilde{Y}=\dot{R}_{s}^{T X}\right| Y=\dot{R}^{T Y}+2 s^{2} \dot{S}^{2} .
$$

Observe that by (1.14), $\sqrt{\pi} \int^{B_{X}} \widehat{e^{m}} \exp \left(-\frac{1}{2} \dot{R}^{T \widetilde{Y}}\right)=\int{ }^{B_{Y}} \exp \left(-\frac{1}{2} \dot{R}^{T Y}\right)=$ $e\left(T Y, \nabla^{T Y}\right)$ is closed. We combine the identity (1.39) with (1.14), (1.19), (1.26), and (1.27) to find

$$
\begin{aligned}
& \sqrt{\pi} \int_{0}^{1} \frac{d s}{s} \mu_{2, s}=\sqrt{\pi} \int_{0}^{1} \frac{d s}{s} d^{V} \int^{B_{X}} \frac{1}{2} \widehat{e}^{\widehat{m}}\left[\exp \left(-\frac{1}{2} j^{*} \dot{R}_{s}^{T X}\right)-\exp \left(-\frac{1}{2} \dot{R}^{T \widetilde{Y}}\right)\right] \\
= & \int_{0}^{1} \frac{d s}{s} d^{V} \int^{B_{Y}} \frac{1}{2}\left[\exp \left(-\frac{1}{2}\left(\dot{R}_{s}^{T X} \mid Y\right)\right)-\exp \left(-\frac{1}{2} \dot{R}^{T Y}\right)\right]=d^{V} B\left(\nabla^{T X}\right) .
\end{aligned}
$$

The proof of Theorem 1.4 is complete.

1.5 The secondary classes for manifolds with boundary. Let $\Omega(W, o(T X)), \Omega(V, o(T X))$ be the $o(T X)$-valued $C^{\infty}$ forms on $W, V$. The algebraic mapping cone of $j^{*}: \Omega(W, o(T X)) \rightarrow \Omega(V, o(T X))$ is defined as the following object: we put

$$
\Omega^{p}(W, V, o(T X))=\Omega^{p}(W, o(T X)) \oplus \Omega^{p-1}(V, o(T X)),
$$

and define the differential by

$$
d\left(\sigma_{1}, \sigma_{2}\right)=\left(d^{W} \sigma_{1}, j^{*} \sigma_{1}-d^{V} \sigma_{2}\right) ;
$$

then the complex $(\Omega(W, V, o(T X)), d)$ calculates the relative cohomology $H^{*}(W, V, o(T X))$ (cf. [BoT, p. 78]). Now we define the relative Euler form of $T X$ associated to $\nabla^{T X}$

$$
E\left(T X, \nabla^{T X}\right):=\left(e\left(T X, \nabla^{T X}\right), e_{b}\left(V, \nabla^{T X}\right)\right) \in \Omega^{m}(W, V, o(T X)) .
$$

Let $g_{0}^{T X}, g_{1}^{T X}$ be two metrics on $T X$, and let $T_{0}^{H} W, T_{1}^{H} W$ be two horizontal sub-bundles of $T W$. Let $\left(g_{s}^{T X}\right)_{s \in \mathbb{R}}$ be a family of metrics connecting $g_{0}^{T X}$ and $g_{1}^{T X}$, with a family $\left(T_{s}^{H} W\right)_{s \in \mathbb{R}}$ of horizontal sub-bundles. We will use the subscript $s$ to distinguish the corresponding objects, like $g_{s}^{T Y}, \nabla_{s}^{T X}$, $\nabla_{s}^{T Y}$, etc.

For the fibration $\pi_{\widetilde{W}}: \widetilde{W}=W \times \mathbb{R} \rightarrow S \times \mathbb{R}$ in subsection 1.3. Let $T^{H}(W \times \mathbb{R})_{s}=T_{s}^{H} W \oplus T \mathbb{R}$, and denote by $g^{T \widetilde{X}}$ the metric on $T \widetilde{X}$ induced 
by $g_{s}^{T X}$. Then by (1.4), $\nabla^{T \widetilde{X}}=\nabla_{s}^{T X}+d s \wedge\left(\frac{\partial}{\partial s}+\frac{1}{2}\left(g_{s}^{T X}\right)^{-1} \frac{\partial}{\partial s} g_{s}^{T X}\right)$, thus

$$
R^{T \widetilde{X}}=p_{W}^{*} R_{s}^{T X}+d s \wedge\left(\frac{\partial}{\partial s} \nabla_{s}^{T X}-\frac{1}{2}\left[\nabla_{s}^{T X},\left(g_{s}^{T X}\right)^{-1} \frac{\partial}{\partial s} g_{s}^{T X}\right]\right) .
$$

Definition 1.8. We define

$$
\begin{gathered}
\widetilde{e}\left(T X, \nabla_{s}^{T X}\right):=\int_{0}^{1} d s i\left(\frac{\partial}{\partial s}\right) e\left(T \widetilde{X}, \nabla^{T \widetilde{X}}\right) \quad \in \Omega^{m-1}(W, o(T X)), \\
\widetilde{e_{b}}\left(V, \nabla_{s}^{T X}\right):=\int_{0}^{1} d s i\left(\frac{\partial}{\partial s}\right) e_{b}\left(V \times \mathbb{R}, \nabla^{T \widetilde{X}}\right) \quad \in \Omega^{m-2}(V, o(T X)), \\
\widetilde{E}\left(T X, \nabla_{0}^{T X}, \nabla_{1}^{T X}\right):=\left(\widetilde{e}\left(T X, \nabla_{s}^{T X}\right),-\widetilde{e_{b}}\left(V, \nabla_{s}^{T X}\right)\right) \in \Omega^{m-1}(W, V, o(T X)) .
\end{gathered}
$$

If $V=\emptyset$, then $\widetilde{E}$ is the Chern-Simons form associated with the Euler class of $T X$, as defined in [BiZ1, (4.53)].

Let $\widetilde{e}\left(T Y, \nabla_{0}^{T Y}, \nabla_{1}^{T Y}\right)$ be the Chern-Simons class of smooth $m$-2-forms on $V$ with values in the orientation line bundle $o(T Y)$, which is defined modulo exact forms, and satisfies

$$
d \widetilde{e}\left(T Y, \nabla_{0}^{T Y}, \nabla_{1}^{T Y}\right)=e\left(T Y, \nabla_{1}^{T Y}\right)-e\left(T Y, \nabla_{0}^{T Y}\right) .
$$

If $\operatorname{dim} X$ is odd, then we derive from (1.18) and (1.45):

$$
\begin{gathered}
\widetilde{e}\left(T X, \nabla_{s}^{T X}\right)=0, \\
2 \widetilde{e_{b}}\left(V, \nabla_{s}^{T X}\right)=\widetilde{e}\left(T Y, \nabla_{0}^{T Y}, \nabla_{1}^{T Y}\right) \text { in } \Omega^{m-2}(V, o(T Y)) / d \Omega(V, o(T Y)) .
\end{gathered}
$$

Theorem 1.9. 1. $E\left(T X, \nabla^{T X}\right)$ is closed in the complex $(\Omega(W, V, o(T X)), d)$ and, modulo exact forms, it does not depend on the choice of $g^{T X}$ and $T^{H} W$, i.e. the cohomology class $E(T X)=\left[E\left(T X, \nabla^{T X}\right)\right] \in H^{m}(W, V, o(T X))$ does not depend on $g^{T X}$ and $T^{H} W$.

2. For a path of metrics on $T X$ and of horizontal sub-bundles of $T W$ as above,

$$
d \widetilde{E}\left(T X, \nabla_{0}^{T X}, \nabla_{1}^{T X}\right)=E\left(T X, \nabla_{1}^{T X}\right)-E\left(T X, \nabla_{0}^{T X}\right) .
$$

Modulo exact forms, $\widetilde{E}\left(T X, \nabla_{0}^{T X}, \nabla_{1}^{T X}\right)$ does not depend on the choice of the paths $g_{s}^{T X}$ and $T_{s}^{H} W$. Thus it defines the secondary relative Euler class of $T X$ in the sense of Chern-Simons.

$$
\begin{aligned}
& \text { 3. We also have } \\
& \int_{0}^{1} d s i\left(\frac{\partial}{\partial s}\right) \mu\left(\nabla^{T \tilde{X}}\right)=B\left(\nabla_{1}^{T X}\right)-B\left(\nabla_{0}^{T X}\right)-d^{V} \int_{0}^{1} d s i\left(\frac{\partial}{\partial s}\right) B\left(\nabla^{T \tilde{X}}\right) .
\end{aligned}
$$

Proof. 1 is a simple application of (1.20a). From Theorem 1.4 we get the first part of 2 ; to get the second part of 2 , we use a deformation argument as in subsection 1.4. Let $g_{s, 1}^{T X}$ (resp. $T_{s, 1}^{H} W$ ) be another family of metrics on $T X$ (resp. sub-bundles of $T W$ ) such that $g_{i, 1}^{T X}=g_{i}^{T X}$, 
$T_{i, 1}^{H} W=T_{i}^{H} W$ for $i=0,1$. Let $\bar{\pi}: \bar{W}=W \times \mathbb{R} \times \mathbb{R} \rightarrow \bar{S}=S \times \mathbb{R} \times \mathbb{R}$ be the fibration with fiber $\bar{X}$ induced by $\pi_{W}: W \rightarrow S$, and let $\bar{p}_{W}$ : $\bar{W} \rightarrow W$ be the natural projection. Let $g_{s, l}^{T X}$ be a family of metrics on $T X$ such that $g_{s, 0}^{T X}=g_{s}^{T X}$ and $g_{s, 1}^{T X}=g_{s, 1}^{T X}, g_{i, l}^{T X}=g_{i}^{T X}$ for $i=0,1$, and in the same way, $T_{s, l}^{H} W$ is a family of paths connecting $T_{s}^{H} W$ and $T_{s, 1}^{H} W$. Set $T^{H} \bar{W}=T_{s, l}^{H} W \oplus T \mathbb{R} \oplus T \mathbb{R}$. Then the construction above applies to the fibration $\bar{\pi}$. Let $\gamma_{1} \in C^{\infty}\left(\bar{W}, \bar{p}_{W}^{*} \Lambda\left(T^{*} W\right) \otimes o(T X)\right)$, $\gamma_{2} \in C^{\infty}\left(V \times \mathbb{R} \times \mathbb{R}, \bar{p}_{W}^{*} \Lambda\left(T^{*} V\right) \otimes o(T X)\right)$ be the coefficients of $d s d l$ in $e\left(T \bar{X}, \nabla^{T \bar{X}}\right)$ and $e_{b}\left(V \times \mathbb{R} \times \mathbb{R}, \nabla^{T \bar{X}}\right)$. Integrating the coefficient of $d s d l$ in the equations $d^{\bar{W}} e\left(T \bar{X}, \nabla^{T \bar{X}}\right)=0$ and $d^{V \times \mathbb{R} \times \mathbb{R}} e_{b}\left(V \times \mathbb{R} \times \mathbb{R}, \nabla^{T \bar{X}}\right)=$ $(j \times \mathrm{Id} \times \mathrm{Id})^{*} e\left(T \bar{X}, \nabla^{T \bar{X}}\right)$ on $[0,1]^{2}$, we get

$$
\begin{gathered}
\widetilde{e}\left(T X, \nabla_{s, 1}^{T X}\right)-\widetilde{e}\left(T X, \nabla_{s, 0}^{T X}\right)=d^{W} \int_{0}^{1} d l \int_{0}^{1} d s \gamma_{1}, \\
\widetilde{e}_{b}\left(V, \nabla_{s, 0}^{T X}\right)-\widetilde{e}_{b}\left(V, \nabla_{s, 1}^{T X}\right)+d^{V} \int_{0}^{1} d l \int_{0}^{1} d s \gamma_{2}=j^{*} \int_{0}^{1} d l \int_{0}^{1} d s \gamma_{1} .
\end{gathered}
$$

This gives the second part of 2 .

From (1.20b), we get $d^{V \times \mathbb{R}} B\left(\nabla^{T \widetilde{X}}\right)=\mu\left(\nabla^{T \widetilde{X}}\right)$. By comparing the coefficient of $d s$, we get $i\left(\frac{\partial}{\partial s}\right) \mu\left(\nabla^{T \tilde{X}}\right)=\frac{\partial}{\partial s} B\left(\nabla_{s}^{T X}\right)-d^{V}\left(i\left(\frac{\partial}{\partial s}\right) B\left(\nabla^{T \tilde{X}}\right)\right)$ which implies (1.49).

REMARK 1.10. Let us make explicit our sign conventions when integrating differential forms along the fiber for the fibration $W \rightarrow S$. If $\gamma$ is a differential form on $S$ and $\left(\sigma_{1}, \sigma_{2}\right) \in \Omega(W, V, o(T X))$ with compact support along the fibers, then

$$
\begin{gathered}
\int_{X}\left(\pi_{W}^{*} \gamma\right) \wedge \sigma_{1}=\gamma\left(\int_{X} \sigma_{1}\right), \quad \int_{Y}\left(\pi_{W}^{*} \gamma\right) \wedge \sigma_{2}=\gamma\left(\int_{Y} \sigma_{2}\right), \\
\int_{(X, Y)}\left(\sigma_{1}, \sigma_{2}\right):=\int_{X} \sigma_{1}+(-1)^{\operatorname{deg} \sigma_{2}-m} \int_{Y} \sigma_{2} .
\end{gathered}
$$

Then (1.51) is compatible with (0.2) in the following sense: if $\left(\sigma_{1}, \sigma_{2}\right) \in$ $\Omega(W, V, o(T X))$ is closed (resp. exact), then $\int_{(X, Y)}\left(\sigma_{1}, \sigma_{2}\right)$ is a closed (resp. exact) form on $S$. In fact, let $d^{X}$ be the exterior differentiation along the fiber $X$, then by [BGV, Prop. 10.1],

$$
\begin{gathered}
d^{S} \int_{Y} \sigma_{2}=\int_{Y} d^{V} \sigma_{2}, \\
d^{S} \int_{X} \sigma_{1}=\int_{X}\left(d^{W} \sigma_{1}-d^{X} \sigma_{1}\right)=\int_{X} d^{W} \sigma_{1}-(-1)^{\operatorname{deg} \sigma_{1}-m+1} \int_{Y} j^{*} \sigma_{1} .
\end{gathered}
$$


This implies that the form $\int_{X} e\left(T X, \nabla^{T X}\right) \omega-(-1)^{\operatorname{deg} \omega} \int_{Y} e_{b}\left(V, \nabla^{T X}\right) \omega$ is closed on $S$, for any closed form $\omega$ on $W$.

\section{Secondary Classes and Mathai-Quillen Form}

In this section, we construct the secondary relative Euler class for manifolds with boundary introduced in section 1, using the Mathai-Quillen form.

This section is organized as follows. In subsection 2.1, we recall the Mathai-Quillen construction of the transgressed Euler forms for Euclidean vector bundles in the formalism of Berezin integrals [BiZ1, §3b)-d)]. In subsection 2.2, we construct the secondary relative Euler class.

2.1 A transgressed Euler class. Let $W$ be a smooth manifold with boundary $V$. Let $\pi_{E}: E \rightarrow W$ be a real vector bundle of dimension $m$. Let $h^{E}$ be a Euclidean metric on $E$, let $\nabla^{E}$ be a Euclidean connection on $\left(E, h^{E}\right)$ with curvature $R^{E}$; then $R^{E}$ is a smooth section of $\Lambda^{2}\left(T^{*} W\right) \otimes \operatorname{End}(E)$. Also, $\pi_{E}^{*} \nabla^{E}$ is a Euclidean connection on $\pi_{E}^{*}\left(E, h^{E}\right)$ with curvature $\pi_{E}^{*} R^{E}$, a smooth section of $\Lambda^{2}\left(T^{*} E\right) \otimes \operatorname{End}\left(\pi_{E}^{*} E\right)$. As in (1.2) and (1.13), we identify $R^{E}$ with $\dot{R}^{E} \in C^{\infty}\left(W, \Lambda^{2}\left(T^{*} W\right) \widehat{\otimes} \widehat{\Lambda^{2} E^{*}}\right)$.

Let $\left\{h_{i}\right\}_{i=1}^{m}$ be an orthonormal basis of $E$ and let $\left\{h^{i}\right\}_{i=1}^{m}$ be the corresponding dual basis of $E^{*}$. The connection $\nabla^{E}$ defines a horizontal subspace $T^{H} E$ of $T E$ such that $T E=T^{H} E \oplus E$. Let $P^{E}$ be the projection $T E \rightarrow E$ and let $P^{E *}: E^{*} \rightarrow T^{*} E$ be the transpose of $P^{E}$. Then $P^{E}$ is a section of $T^{*} E \otimes E$ and $P_{Z}^{E}=\left(\pi_{E}^{*} \nabla^{E}\right) Z$. Denote by $E \ni Z \rightarrow Z^{b} \in E^{*}$ the isomorphism induced by the metric $h^{E}$, then $P^{E}$ will be identified with the section of $T^{*} E \widehat{\otimes} \widehat{E^{*}}$ given by $\dot{P}^{E}=\sum_{i=1}^{m}\left(P^{E *} h^{i}\right) \otimes \widehat{h^{i}}$.

Definition 2.1. For $t>0$, let $A_{t}$ be the section of $\Lambda\left(T^{*} E\right) \widehat{\otimes} \pi_{E}^{*} \widehat{\Lambda E^{*}}$ on $E$ given by

$$
A_{t}(Z)=\frac{1}{2} \pi_{E}^{*} \dot{R}^{E}+\sqrt{t} \dot{P}_{Z}^{E}+t|Z|^{2} \quad \text { for all } Z \in E .
$$

We will apply the formalism of Berezin integrals, summarized in subsection 1.1, to $T E$ : If $\omega$ is a smooth section of $\Lambda\left(T^{*} E\right) \widehat{\otimes} \pi_{E}^{*} \widehat{\Lambda E^{*}}, \int^{B} \omega$ is a smooth section of $\Lambda\left(T^{*} E\right) \widehat{\otimes} \pi_{E}^{*} o(E)$, i.e. a smooth differential form on $E$ with values in $\pi_{E}^{*} o(E)$. Set $e\left(E, \nabla^{E}\right):=\operatorname{Pf}\left[R^{E} / 2 \pi\right]$. Then $e\left(E, \nabla^{E}\right)$ is a smooth closed section of $\Lambda^{\operatorname{dim} E}\left(T^{*} W\right) \widehat{\otimes} o(E)$. If $V=\emptyset, e\left(E, \nabla^{E}\right)$ is a Chern-Weil representative of the rational Euler class of $E$. Of course, if $\operatorname{dim} E$ is odd, then $e\left(E, \nabla^{E}\right)=0$. 
Definition 2.2. We define two differential forms on $E$ with values in $\pi_{E}^{*} O(E)$ by

$$
a_{t}(Z)=\int^{B} \exp \left(-A_{t}(Z)\right), \quad b_{t}(Z)=\int^{B} \frac{\widehat{Z^{b}}}{2 \sqrt{t}} \wedge \exp \left(-A_{t}(Z)\right) .
$$

Let $\int_{E}$ denote the fiber integral of forms on $E$ taking values in $\pi_{E}^{*} o(E)$. Now we state an important result of Mathai and Quillen [MQ, Thm.6.4] (also cf. [BiZ1, Thms. 3.4, 3.5]).

Theorem 2.3. The forms $a_{t}$ are closed of degree $m$. For $t>0$, the forms $a_{t}$ represent the Thom class of $E$, such that $\int_{E} a_{t}=1$. Moreover, for $t>0$ we have the relations

Then

$$
\begin{gathered}
a_{0}=\pi_{E}^{*} e\left(E, \nabla^{E}\right), \\
b_{t}=-\frac{1}{2 t} i(Z) a_{t}, \quad \frac{\partial a_{t}}{\partial t}=-d b_{t} .
\end{gathered}
$$

$$
\psi\left(E, \nabla^{E}\right):=\lim _{\varepsilon \rightarrow 0} \int_{\varepsilon}^{1 / \varepsilon} b_{t} d t
$$

exists as an $(m-1)$-form on $E \backslash\{0\}$ with values in $\pi_{E}^{*} o(E)$.

Definition 2.4. The form $\psi\left(E, \nabla^{E}\right)$ is called the Mathai-Quillen form.

In fact, $\psi\left(E, \nabla^{E}\right)$ is defined on $E$ as a current (cf. [BiZ1, Thm. 3.7]) and

$$
\begin{gathered}
d \psi\left(E, \nabla^{E}\right)=\pi_{E}^{*} e\left(E, \nabla^{E}\right)-\delta_{W}, \\
\psi\left(E, \nabla^{E}\right)(\lambda Z)=(\operatorname{sign} \lambda)^{m} \psi\left(E, \nabla^{E}\right)(Z), \text { for } \lambda \in \mathbb{R} \backslash\{0\} .
\end{gathered}
$$

REMARK 2.5. If $E=T W$, then the restriction of $\psi\left(E, \nabla^{E}\right)$ to the sphere bundle $S(E)$ of $E$ coincides with the transgressed Euler class defined by Chern (cf. [Che2], [Z, §3.5]).

The following result was essentially obtained in [BiZ1, (6.20)].

Proposition 2.6. Let $j_{S}: S(E) \rightarrow E$ be the natural injection. Let $E^{\perp} \subset \pi_{E}^{*} E$ be the relative tangent bundle of the fibration $\pi_{S}: S(E) \rightarrow W$. We identify $o\left(E^{\perp}\right)$ to $\pi_{S}^{*} o(E)$ as in (1.14), in viewing $S(E)$ as the boundary of the unit ball $D(E)$ on each fiber. Let $\nabla^{E^{\perp}}$ be the connection on $E^{\perp}$ induced by $\pi_{E}^{*} \nabla^{E}$ by taking the orthogonal projection. If $\operatorname{dim} E$ is odd, then on $S(E)$,

$$
j_{S}^{*} \psi\left(E, \nabla^{E}\right)=\frac{1}{2} e\left(E^{\perp}, \nabla^{E^{\perp}}\right) .
$$

Proof. Let $L \subset \pi_{E}^{*} E$ be the tautological line bundle on $S(E)$, then $E^{\perp}$ is the orthogonal complement of $L$ in $\left(\pi_{S}^{*} E, h_{S}^{\pi_{S}^{*} E}\right)$. Let $P^{L}, P^{E^{\perp}}$ be the orthogonal projections from $\left(\pi_{S}^{*} E, h^{\pi_{S}^{*}}\right)$ onto $L, E^{\perp}$, then $\nabla^{E^{\perp}}=P^{E^{\perp}} \pi_{S}^{*} \nabla^{E}$. Set $\nabla^{L}=P^{L} \pi_{S}^{*} \nabla^{E}, A=\pi_{S}^{*} \nabla^{E}-\left(\nabla^{E^{\perp}} \oplus \nabla^{L}\right)$, then $A$ is a one-form on $S(E)$ 
taking values in the skew-adjoint endomorphisms of $\pi_{S}^{*} E$ which exchange $E^{\perp}$ and $L$. Let $\sigma_{L}$ be the tautological section of $\pi_{S}^{*} E$ such that $-\sigma_{L}$ is the inward pointing unit vector of $S(E)$ in $D(E)$. Then $j_{S}^{*} P^{E}=\pi_{S}^{*} \nabla^{E} \sigma_{L}=$ $A \sigma_{L} \in T^{*} S(E) \otimes E^{\perp}$. Set $\dot{S}_{\sigma_{L}}=\frac{1}{2} \sum_{j} g^{j} \wedge\left(\widehat{A\left(g_{j}\right) \sigma_{L}}\right)^{b} \in T^{*} S(E) \widehat{\otimes} \widehat{E^{\perp *}}$, with $\left\{g_{j}\right\}$ an orthonormal basis of $T S(E)$ and $\left\{g^{j}\right\}$ its dual basis. As in (1.16), we have

$$
\dot{R}^{E^{\perp}}=\pi_{S}^{*} \dot{R}^{E}-2 \dot{S}_{\sigma_{L}}^{2}+a \wedge \widehat{\left(\sigma_{L}\right)^{b}} .
$$

Now by (1.14), (2.2), (2.4) and (2.7), if $\operatorname{dim} E$ is odd,

$$
\begin{aligned}
j_{S}^{*} \psi\left(E, \nabla^{E}\right) & =\int_{0}^{\infty} \frac{d t}{2 \sqrt{t}} \int^{B} \widehat{\left(\sigma_{L}\right)^{b}} \exp \left(-\frac{1}{2} \pi_{S}^{*} \dot{R}^{E}-2 \sqrt{t} \dot{S}_{\sigma_{L}}-t\right) \\
& =\int^{B} \widehat{\left(\sigma_{L}\right)^{b}} \exp \left(-\frac{1}{2} \pi_{S}^{*} \dot{R}^{E}\right) \sqrt{\pi} \sum_{k=0}^{\infty} \frac{\left(-\dot{S}_{\sigma_{L}}\right)^{k}}{2 \Gamma\left(\frac{k}{2}+1\right)} \\
& =\frac{\sqrt{\pi}}{2} \int^{B} \widehat{\left(\sigma_{L}\right)^{b}} \exp \left(-\frac{1}{2} \dot{R}^{E^{\perp}}\right)=\frac{1}{2} e\left(E^{\perp}, \nabla^{E^{\perp}}\right) .
\end{aligned}
$$

2.2 Secondary relative Euler class. In this subsection, we assume that there exists a non-vanishing section, $\sigma$, of $E$ on $V$; we assume $|\sigma|_{h^{E}}=1$. Then it follows from (2.5), that on $V$,

$$
d \sigma^{*} \psi\left(E, \nabla^{E}\right)=j^{*} e\left(E, \nabla^{E}\right) .
$$

Let $(\Omega(W, V, o(E)), d)$ be the algebraic mapping cone of $j^{*}$, with $j^{*}$ as in subsection 1.5, but with $o(T X)$ replaced by $o(E)$. Thus we will consider

$$
E_{\sigma}\left(E, \nabla^{E}\right):=\left(e\left(E, \nabla^{E}\right), \sigma^{*} \psi\left(E, \nabla^{E}\right)\right) \in H^{m}(W, V, o(E)),
$$

as the Chern-Weil representative associated to the relative Euler class of $\left(E, \nabla^{E}\right)$.

Let $\left(h_{0}^{E}, \nabla_{0}^{E}\right),\left(h_{1}^{E}, \nabla_{1}^{E}\right)$ be two pairs of metrics and Euclidean connections on $E$. Let $\left(h_{s}^{E}\right)_{s \in \mathbb{R}}$ be a smooth family of metrics on $E$ connecting $h_{0}^{E}, h_{1}^{E}$. Let $p_{W}: W \times \mathbb{R} \rightarrow W$ be the natural projection such that $\left(\widetilde{E}=p_{W}^{*} E, h_{\mid W \times\{s\}}^{\widetilde{E}}=h_{s}^{E}\right)$ is a Euclidean vector bundle on $W \times \mathbb{R}$. The section $\sigma$ induces naturally a normalized section of $\left.\left(\widetilde{E}, h^{\widetilde{E}}\right)\right|_{V \times \mathbb{R}}$ which we denote by $\widetilde{\sigma}$. Choose a Euclidean connection $\nabla^{\widetilde{E}}$ on $\left(\widetilde{E}, h^{\widetilde{E}}\right)$ such that $\nabla_{\mid W \times\{0\}}^{\widetilde{E}}=\nabla_{0}^{E}, \nabla_{\mid W \times\{1\}}^{\widetilde{E}}=\nabla_{1}^{E}$ and denote by $\nabla_{s}^{E}$ its restriction to $W \times\{s\}$. We set (cf. (1.45))

$$
\begin{gathered}
\widetilde{e}\left(E, \nabla_{s}^{E}\right):=\int_{0}^{1} d s i\left(\frac{\partial}{\partial s}\right) e\left(\widetilde{E}, \nabla^{\widetilde{E}}\right) \quad \in \Omega^{m-1}(W, o(E)), \\
\widetilde{e_{b, \sigma}}\left(V, \nabla_{s}^{E}\right):=\int_{0}^{1} d s i\left(\frac{\partial}{\partial s}\right) \widetilde{\sigma}^{*} \psi\left(\widetilde{E}, \nabla^{\widetilde{E}}\right) \quad \in \Omega^{m-2}(V, o(E)), \\
\widetilde{E}_{\sigma}\left(E, \nabla_{0}^{E}, \nabla_{1}^{E}\right):=\left(\widetilde{e}\left(E, \nabla_{s}^{E}\right),-\widetilde{e_{b, \sigma}}\left(V, \nabla_{s}^{E}\right)\right) \quad \in \Omega^{m-1}(W, V, o(E)) .
\end{gathered}
$$


Proposition 2.7. The cohomology class $E_{\sigma}\left(E, \nabla^{E}\right)$ in $H^{m}(W, V, o(E))$ does not depend on the choice of $\left(\nabla^{E}, h^{E}\right)$. Moreover, for a path $\left(\nabla_{s}^{E}, h_{s}^{E}\right)$ as above, we have

$$
d \widetilde{E}_{\sigma}\left(E, \nabla_{0}^{E}, \nabla_{1}^{E}\right)=E_{\sigma}\left(E, \nabla_{1}^{E}\right)-E_{\sigma}\left(E, \nabla_{0}^{E}\right) .
$$

Modulo exact forms, $\widetilde{E}_{\sigma}\left(E, \nabla_{0}^{E}, \nabla_{1}^{E}\right)$ does not depend on the choice of $\left(\nabla_{s}^{E}, h_{s}^{E}\right)_{s \in[0,1]}$. Thus $\widetilde{E}_{\sigma}$ defines the secondary relative Euler class of $E$ in the sense of Chern-Simons.

Proof. Since $e\left(\widetilde{E}, \nabla^{\widetilde{E}}\right)$ is closed on $W \times \mathbb{R}$, we have

$$
d^{W} \widetilde{e}\left(E, \nabla_{s}^{E}\right)=e\left(E, \nabla_{1}^{E}\right)-e\left(E, \nabla_{0}^{E}\right) \text {. }
$$

By (2.9), we have $d^{V \times \mathbb{R}} \widetilde{\sigma}^{*} \psi\left(\widetilde{E}, \nabla^{\widetilde{E}}\right)=(j \times \mathrm{Id})^{*} e\left(\widetilde{E}, \nabla^{\widetilde{E}}\right)$, hence by comparing the coefficient of $d s$ we get

$$
\frac{\partial}{\partial s} \widetilde{\sigma}^{*} \psi\left(E, \nabla_{s}^{E}\right)-d^{V}\left[i\left(\frac{\partial}{\partial s}\right) \widetilde{\sigma}^{*} \psi\left(\widetilde{E}, \nabla^{\widetilde{E}}\right)\right]=(j \times \mathrm{Id})^{*}\left[i\left(\frac{\partial}{\partial s}\right) e\left(\widetilde{E}, \nabla^{\widetilde{E}}\right)\right] .
$$

Integrating both sides over $s \in[0,1]$, we get

$$
j^{*} \widetilde{e}\left(E, \nabla_{s}^{E}\right)+d^{V} \widetilde{e}_{b, \sigma}\left(E, \nabla_{s}^{E}\right)=\sigma^{*} \psi\left(E, \nabla_{1}^{E}\right)-\sigma^{*} \psi\left(E, \nabla_{0}^{E}\right),
$$

which together with (2.13) implies (2.12). To get the last part of Proposition 2.7, we use (2.9) and a deformation argument as in the proof of Theorem 1.9.

REMARK 2.8. The classes $E_{\sigma}, \widetilde{E}_{\sigma}$ depend on the section $\sigma$, in fact $E_{-\sigma}=-E_{\sigma}$, if $\operatorname{dim} E$ is odd. But in the situation of subsection 1.2, there exists a natural choice of the unit section $\sigma$, normally $e_{\mathfrak{n}}$; thus Proposition 2.7 is applicable.

Set $\dot{S}_{\sigma}=\frac{1}{2} \sum_{j} f^{j} \wedge\left(\widehat{\left.\nabla_{f_{j}}^{E} \sigma\right)^{b}} \in T^{*} W \widehat{\otimes} \widehat{E^{*}}\right.$ with $\left\{f_{j}\right\}$ a basis of $T W$ and $\left\{f^{j}\right\}$ its dual basis. From Definition 2.1, for $t \geq 0, \sigma^{*} A_{t}=\frac{1}{2} \dot{R}^{E}+2 \sqrt{t} \dot{S}_{\sigma}+t$. By (2.4), as in (2.8),

$$
\sigma^{*} \psi\left(E, \nabla^{E}\right)=\sqrt{\pi} \int^{B} \widehat{\sigma^{b}} \exp \left(-\frac{1}{2} j^{*} \dot{R}^{E}\right) \sum_{k=0}^{\infty} \frac{\left(-\dot{S}_{\sigma}\right)^{k}}{2 \Gamma\left(\frac{k}{2}+1\right)} .
$$

Now if we take $\sigma=e_{\mathfrak{n}}$, then by (1.15), $\dot{S}=\dot{S}_{\sigma}$. By (1.14), (1.17), and (2.16), we thus get the following result.

Proposition 2.9. With the notation of section 1 we have

$$
e_{\mathfrak{n}}^{*} \psi\left(T X, \nabla^{T X}\right)=e_{b}\left(V, \nabla^{T X}\right) \text {. }
$$

\section{Local Gauss-Bonnet-Chern Theorem}

In this section, we establish a local version of the Gauss-Bonnet-Chern theorem for manifolds with boundary using heat-kernel methods. We do not assume that the metric has product structure near the boundary. 
This section is organized as follows. In subsection 3.1, we state the Gauss-Bonnet-Chern theorem for manifolds with boundary and in subsections $3.2-3.8$ we prove a local version. In subsection 3.2 , using [BiZ1, §4h)] and a key estimate established in [RS, Lemma 5.12] (also cf. [Gr, (2.4.22)]), we reduce the problem to a problem near the boundary $Y$ of $X$, and we explain that we can localize the problem. In subsection 3.3, we recall the Lichnerowicz formula from [BiZ1, Thm. 4.13]. In subsection 3.4, we reformulate our boundary condition and in subsection 3.5, we construct a trivialization and rescale the coordinate $Z \in T_{y_{0}} X$; we also use the Bismut-Zhang rescaling on Clifford variables along the boundary $Y$. Moreover we calculate the limits of the operators and the boundary conditions. In subsection 3.6, we establish uniform estimates on the heat kernels of the rescaled operators with suitable boundary conditions. In subsection 3.7, we obtain the explicit fundamental solution of our model problem. In subsection 3.8, we use the result of subsection 3.7 to prove Theorem 3.2, the local Gauss-BonnetChern theorem.

We use the notation in subsection 1.3.

3.1 The Gauss-Bonnet-Chern theorem. Let $X$ be a compact smooth manifold with boundary $Y$ and dimension $\operatorname{dim} X=m$, and let $F$ be a flat complex vector bundle over $X$ with flat connection $\nabla^{F}$.

Denote by $\Omega(X, F):=\oplus_{p=0}^{m} \Omega^{p}(X, F):=\oplus_{p=0}^{m} C^{\infty}\left(X, \Lambda^{p}\left(T^{*} X\right) \otimes F\right)$ the space of smooth differential forms on $X$ with values in $F$. The bundle $\Lambda\left(T^{*} X\right)$ is $\mathbb{Z}$-graded, and so it possesses a natural $\mathbb{Z}_{2}$-grading. The flat connection $\nabla^{F}$ extends naturally to a differential, $d^{F}$, on $\Omega(X, F)$. The cohomology of the complex $\left(\Omega(X, F), d^{F}\right)$ is called the (absolute) de-Rham cohomology of $X$ with coefficients in $F$, and is denoted by $H^{\bullet}(X, F)$. Define the Euler characteristic of $F$ and $X$, respectively, by

$$
\chi(X, F):=\sum_{p=0}^{m}(-1)^{p} \operatorname{dim}_{\mathbb{C}} H^{p}(X, F), \quad \chi(X):=\chi(X, \mathbb{C}) .
$$

Consider next an arbitrary Riemannian metric $g^{T X}$ on $X$ and an arbitrary Hermitian metric $h^{F}$ on $F$, and denote by $\langle\cdot, \cdot\rangle_{\Lambda\left(T^{*} X\right) \otimes F}$ the induced Hermitian metric on $\Lambda\left(T^{*} X\right) \otimes F$, and let $d v_{X}$ be the Riemannian volume element on $\left(T X, g^{T X}\right)$, then we can view $d v_{X}$ as a section of $\Lambda^{m}\left(T^{*} X\right) \otimes o(T X)$. We define a Hermitian product on $\Omega(X, F)$ by

$$
\left\langle\sigma, \sigma^{\prime}\right\rangle:=\int_{X}\left\langle\sigma, \sigma^{\prime}\right\rangle_{\Lambda\left(T^{*} X\right) \otimes F} d v_{X},
$$

for $\sigma, \sigma^{\prime} \in \Omega(X, F)$; the Hilbert space obtained by completion is denoted by $L^{2}\left(X, \Lambda\left(T^{*} X\right) \otimes F\right)$. We consider $d^{F}$ as an unbounded operator in 
$L^{2}\left(X, \Lambda\left(T^{*} X\right) \otimes F\right)$ with domain $\Omega_{0}(X, F):=\{\sigma \in \Omega(X, F)$; supp $\sigma \cap Y=\emptyset\}$. The adjoint operator $d^{F *}$ is also defined on $\Omega_{0}(X, F)$. Let $F^{*}$ be the dual of $F$. Then $\nabla^{F}$ defines a connection $\nabla^{F}$ on $F^{*}$ by the identification $F \rightarrow F^{*}$ induced by the metric $h^{F}$. We denote by $d^{F \otimes o(T X)}$ the differential on $\Omega\left(X, F^{*} \otimes o(T X)\right)$ induced by the flat connection $\nabla^{F}$ on $F^{*}$. Let $*^{F}$ be the Hodge operator on $*^{F}: \Lambda\left(T^{*} X\right) \otimes F \rightarrow \Lambda\left(T^{*} X\right) \otimes F^{*} \otimes o(T X)$ defined by $\left(\sigma \wedge *^{F} \sigma^{\prime}\right)_{F}:=\left\langle\sigma, \sigma^{\prime}\right\rangle_{\Lambda\left(T^{*} X\right) \otimes F} d v_{X}$. Then on $\Omega^{p}(X, F)$,

$$
d^{F *}=(-1)^{p}\left(*^{F}\right)^{-1} d^{F \otimes o(T X)} *^{F},
$$

and so is

$$
D:=d^{F}+d^{F *} .
$$

Then $D^{2}=d^{F} d^{F *}+d^{F *} d^{F}: \Omega^{p}(X, F) \rightarrow \Omega^{p}(X, F)$ is the Hodge Laplacian associated to the pair of metrics $g^{T X}$ and $h^{F}$.

Next we need to define self-adjoint extensions of $D$ by elliptic boundary conditions. To do so, we use the metric on $X$ to identify the normal bundle $N$ to $Y$ in $X$ with the orthogonal complement of $T Y$ in $\left.T X\right|_{Y}$. Denote then by $e_{\mathfrak{n}}$ the inward pointing unit normal vector field along $Y$. Then we put with $i(\cdot)$ interior multiplication

$$
\begin{gathered}
\Omega_{a}^{p}(X, F):=\left\{\sigma \in \Omega^{p}(X, F) ; i\left(e_{\mathfrak{n}}\right) \sigma=i\left(e_{\mathfrak{n}}\right)\left(d^{F} \sigma\right)=0 \text { on } Y\right\} ; \\
D_{a}:=D\left|\Omega_{a}(X, F):=D\right| \oplus_{p=0}^{m} \Omega_{a}^{p}(X, F) ; \\
H_{a}^{p}(X, F):=\operatorname{Ker} D_{a} \cap \Omega_{a}^{p}(X, F) .
\end{gathered}
$$

Note that $D_{a}$ is essentially self-adjoint.

Theorem 3.1. We have a canonical isomorphism

$$
H_{a}^{p}(X, F) \simeq H^{p}(X, F) .
$$

Proof. If $h^{F}$ is flat, (3.6) was proved in [RS, Prop.4.2], (cf. also [BrüL, Thm. 4.2], [G, Thm.2.7.3], [Mü1, p. 239]), but the same proof works in the general case.

Recall that $\nabla^{T X}$ is the Levi-Civita connection on $\left(T X, g^{T X}\right)$ and that the forms $e\left(T X, \nabla^{T X}\right), e\left(T Y, \nabla^{T Y}\right)$ and $e_{b}\left(Y, \nabla^{T X}\right)$ are defined in subsection 1.3. In the case $Y=\emptyset$, the Gauss-Bonnet-Chern theorem [Che2] gives

$$
\chi(X, F)=\operatorname{rk}(F) \int_{X} e\left(T X, \nabla^{T X}\right) .
$$

If $Y \neq \emptyset$, then $e_{b}\left(Y, \nabla^{T X}\right)$ is an $o(T Y)$-valued $m-1$-form on $Y$. If $m$ is odd, then

$$
e\left(T X, \nabla^{T X}\right)=0, \quad e_{b}\left(Y, \nabla^{T X}\right)=\frac{1}{2} e\left(T Y, \nabla^{T Y}\right) .
$$

Thus we obtain a reformulation of the Gauss-Bonnet-Chern theorem $[\mathrm{Ch} 1,2],[\mathrm{G}, \S 2.7]$ in terms of Berezin integrals. This result is essentially 
contained in the work of Chern, but we will give a new derivation by establishing the corresponding local index theorem in the remainder of this section.

Theorem 3.2 (Gauss-Bonnet-Chern).

$$
\begin{aligned}
\chi(X, F) & =\operatorname{rk}(F) \int_{X} e\left(T X, \nabla^{T X}\right)+(-1)^{m-1} \operatorname{rk}(F) \int_{Y} e_{b}\left(Y, \nabla^{T X}\right) \\
& =(-1)^{m} \operatorname{rk}(F) \int_{(X, Y)} E\left(T X, \nabla^{T X}\right),
\end{aligned}
$$

using the notation of $(0.2)$ in the last identity.

Let $\exp \left(-t D_{a}^{2}\right)$ be the heat semi-group of $D_{a}^{2}$, with $D_{a}$ from (3.5). By the McKean-Singer formula, for $t>0$ we have

$$
\chi(X, F)=\operatorname{Tr}_{s}\left[\exp \left(-t D_{a}^{2}\right)\right]=\sum_{p=0}^{m}(-1)^{p} \operatorname{Tr}_{\Omega_{a}^{p}(X, F)}\left[\exp \left(-t D_{a}^{2}\right)\right] .
$$

We will prove Theorem 3.2 by computing the right-hand side of (3.9).

3.2 Localization of the problem near the boundary $\boldsymbol{Y}$. Let $d$ be the distance function on $\left(X, g^{T X}\right)$. For $x \in X$, let $d(x, Y)=\inf _{y \in Y} d(x, y)$ denote the distance of the point $x$ to the boundary $Y$. Let $e^{-t D_{a}^{2}}\left(x, x^{\prime}\right)$, $\left(x, x^{\prime} \in X\right)$, be the kernel of $e^{-t D_{a}^{2}}$ with respect to $d v_{X}\left(x^{\prime}\right)$.

We may and will assume that $X$ is embedded in a $m$-dimensional closed smooth manifold $X^{\prime}$, equipped with a flat complex vector bundle $F^{\prime}$, with flat connection $\nabla^{F^{\prime}}$, and metrics $g^{T X^{\prime}}$ and $h^{F^{\prime}}$, in such a way that the data on $X$ are obtained by restricting the data on $X^{\prime}$ to $X$. For $0<\varepsilon<\infty$, set $U_{\varepsilon}=Y \times(-\varepsilon, \varepsilon)$. For $\varepsilon_{0}$ small enough, the map $U_{2 \varepsilon_{0}} \ni\left(y, x_{m}\right) \mapsto \exp _{y}^{X^{\prime}}\left(x_{m} e_{\mathfrak{n}}(y)\right) \in X^{\prime}$ is a diffeomorphism from $U_{2 \varepsilon_{0}}$ onto a tubular neighborhood $\mathcal{U}_{2 \varepsilon_{0}}$ of $Y$ in $X^{\prime}$. We identify $\mathfrak{U}_{2 \varepsilon_{0}}$ with $Y \times\left(-2 \varepsilon_{0}, 2 \varepsilon_{0}\right)$ and use the notation $x=\left(y, x_{m}\right)$, such that $Y \times\left[0,2 \varepsilon_{0}\right) \subset X$.

Let $d v_{N}=e^{m}$ be the Euclidean volume form on $N$, and let $d v_{Y}$ be the Riemannian volume form on $\left(Y, g^{T Y}\right)$. For $y \in Y, x_{m} \in \mathbb{R},\left|x_{m}\right| \leq \varepsilon_{0}$, let $\kappa\left(y, x_{m}\right)$ be the function defined by

$$
d v_{X}\left(y, x_{m}\right)=: \kappa\left(y, x_{m}\right) d v_{N}\left(x_{m}\right) d v_{Y}(y)
$$

note that $\kappa(y, 0)=1$.

Denote by $D^{\prime}$ the analogue of $D$ on $X^{\prime}$ and let $e^{-t D^{\prime 2}}\left(x, x^{\prime}\right)$ be the kernel of $\exp \left(-t D^{\prime 2}\right)$ with respect to $d v_{X^{\prime}}\left(x^{\prime}\right)$. For $x, x^{\prime} \in X, y \in Y$, set

$$
\begin{gathered}
P_{t}\left(x, x^{\prime}\right)=\left(e^{-t D_{a}^{2}}-e^{-t D^{\prime 2}}\right)\left(x, x^{\prime}\right), \\
I_{t}(y)=\int_{0}^{t^{1 / 4}} \operatorname{Tr}_{s}\left[P_{t}\left(\left(y, x_{m}\right),\left(y, x_{m}\right)\right)\right] \kappa\left(y, x_{m}\right) d v_{N}\left(x_{m}\right) .
\end{gathered}
$$


Then by [RS, Lemma 5.12] (also cf. [Gr, (2.4.22)]), there exist constants $c, C>0$ such that for $x, x^{\prime} \in X, t \in(0,1]$,

$$
\left|P_{t}\left(x, x^{\prime}\right)\right| \leq C t^{-m / 2} \exp \left(-\frac{c}{t}\left(d^{2}(x, Y)+d^{2}\left(x^{\prime}, Y\right)+d^{2}\left(x, x^{\prime}\right)\right)\right) .
$$

Now, by [BGV, Thm.4.1] (cf. also [BiZ1, §4h)]) we have as $t \rightarrow 0$, uniformly on $X^{\prime}$,

$$
\operatorname{Tr}_{s}\left[e^{-t D^{\prime 2}}(x, x)\right] d v_{X}(x)=\operatorname{rk}(F) e\left(T X, \nabla^{T X}\right)(x)+\mathcal{O}\left(t^{1 / 2}\right) .
$$

Thus we get from (3.9), (3.11), (3.12), and (3.13), as $t \rightarrow 0$,

$$
\begin{aligned}
\chi(X, F) & =\int_{X} \operatorname{Tr}_{s}\left[e^{-t D_{a}^{2}}(x, x)\right] d v_{X}(x) \\
& =\int_{X} \operatorname{Tr}_{s}\left[e^{-t D^{\prime 2}}(x, x)\right] d v_{X}(x)+\int_{X} \operatorname{Tr}_{s}\left[P_{t}(x, x)\right] d v_{X}(x) \\
& =\operatorname{rk}(F) \int_{X} e\left(T X, \nabla^{T X}\right)+\mathcal{O}\left(t^{1 / 2}\right)+\int_{X \cap U_{t^{1 / 4}}} \operatorname{Tr}_{s}\left[P_{t}(x, x)\right] d v_{X}(x) \\
& =\operatorname{rk}(F) \int_{X} e\left(T X, \nabla^{T X}\right)+\int_{Y} I_{t}(y) d v_{Y}(y)+\mathcal{O}\left(t^{1 / 2}\right) .
\end{aligned}
$$

To prove Theorem 3.2, it is hence enough to calculate the limit of $I_{t}(y)$ when $t \rightarrow 0$.

Lemma 3.3. The limit of $I_{t}(y)$ when $t \rightarrow 0$ only depends on the restriction of $D^{2}$ to $B_{\varepsilon_{0}}^{X}(y)$.

Proof. Let $f$ be a smooth even function defined on $\mathbb{R}$ with values in $[0,1]$, such that $f(u)=1$ for $|u| \leq \varepsilon_{0} / 2$, and $f(u)=0$ for $|u| \geq \varepsilon_{0}$. Set $g(u)=$ $1-f(u)$. For $t \in(0,1], a \in \mathbb{C}$, set

$$
\begin{aligned}
& F_{t}(a)=\int_{-\infty}^{+\infty} \exp (i u a \sqrt{2}) \exp \left(\frac{-u^{2}}{2}\right) f(u t) \frac{d u}{\sqrt{2 \pi}}, \\
& H_{t}(a)=\int_{-\infty}^{+\infty} \exp (i u a \sqrt{2}) \exp \left(-\frac{u^{2}}{2 t^{2}}\right) g(u) \frac{d u}{t \sqrt{2 \pi}},
\end{aligned}
$$

such that $F_{t}(a)+H_{t}(a / t)=\exp \left(-a^{2}\right)$. The functions $F_{t}(a), H_{t}(a)$ are even holomorphic functions, so there exist holomorphic functions $\widetilde{F}_{t}(a), \widetilde{H}_{t}(a)$ such that $F_{t}(a)=\widetilde{F}_{t}\left(a^{2}\right)$ and $H_{t}(a)=\widetilde{H}_{t}\left(a^{2}\right)$. Thus

$$
\widetilde{F}_{t}\left(t^{2} D_{a}^{2}\right)+\widetilde{H}_{t}\left(D_{a}^{2}\right)=e^{-t^{2} D_{a}^{2}} .
$$

Let $\widetilde{H}_{t}\left(D_{a}^{2}\right)(x, z), \widetilde{F}_{t}\left(t^{2} D_{a}^{2}\right)(x, z)(x, z \in X)$, be the smooth kernels of $\widetilde{H}_{t}\left(D_{a}^{2}\right)$ and $\widetilde{F}_{t}\left(t^{2} D_{a}^{2}\right)$ with respect to $d v_{X}(z)$.

By the definition, there exist a positive constant $c$ such that for any $k \in \mathbb{N}$, there exists $C_{k}>0$, such that

$$
\sup _{a \in \mathbb{R}}|a|^{k}\left|H_{t}(a)\right| \leq C_{k} e^{-c / t^{2}}
$$


Thus for any $k, l \in \mathbb{N}$,

$$
\left|D_{a}^{2 l} \widetilde{H}_{t}\left(D_{a}^{2}\right) D_{a}^{2 k}\right|_{L^{2}} \leq C_{k, l} e^{-c / t^{2}} .
$$

Moreover, $D_{x}^{2 l} D_{z}^{2 k} \widetilde{H}_{t}\left(D_{a}^{2}\right)(x, z)$ verifies the boundary condition for $x, z$ separately.

By elliptic estimate and Sobolev inequality, we get immediately the following estimate for any $x, z \in X$,

$$
\left|\widetilde{H}_{t}\left(D_{a}^{2}\right)(x, z)\right| \leq C e^{-c / t^{2}} .
$$

Now we employ finite propagation speed of hyperbolic operators, as explained in $[\mathrm{T}, \S 2.8, \S 6.1]$; it follows that for $x, z \in X$ with $d(x, z) \geq \varepsilon_{0}$, we have

$$
\widetilde{F}_{t}\left(t^{2} D_{a}^{2}\right)(x, z)=0 .
$$

Moreover, by the same token we see that, given $x \in X, \widetilde{F}_{t}\left(t^{2} D_{a}^{2}\right)(x, \cdot)$ depends only on the restriction of $D^{2}$ to $B_{\varepsilon_{0}}^{X}(x)$. By (3.16), (3.19), and (3.20), we get Lemma 3.3.

3.3 Clifford algebras and the Lichnerowicz formula. For $X \in T X$, denote by $X^{b} \in T^{*} X$ the one form corresponding to $X$ under the metric $g^{T X}$. Set

$$
c(X):=w\left(X^{b}\right)-i(X), \quad \widehat{c}(X):=w\left(X^{b}\right)+i(X) .
$$

Then for $U, V \in T X$, we have the Clifford relations:

$$
\begin{aligned}
& c(U) c(V)+c(V) c(U)=-2\langle U, V\rangle, \\
& \widehat{c}(U) \widehat{c}(V)+\widehat{c}(V) \widehat{c}(U)=2\langle U, V\rangle, \\
& c(U) \widehat{c}(V)+\widehat{c}(V) c(U)=0 .
\end{aligned}
$$

From (3.22) we deduce that the maps $T X \ni e \mapsto c(e), \widehat{c}(e)$ extend to representations of the respective Clifford algebras. Also, $\operatorname{End}\left(\Lambda\left(T^{*} X\right)\right)$ is generated by the endomorphisms $c(e)$ and $\widehat{c}(e)$ for $e \in T X$. In particular, if $B \in \operatorname{End}(T X)$ is antisymmetric, then the action of $B$ on $\Lambda\left(T^{*} X\right)$ as a derivation (cf. $[\mathrm{BGV},(1.26)]$ ) is given by

$$
\sum_{i, j}\left\langle e_{j}, B e_{i}\right\rangle w\left(e^{j}\right) i\left(e_{i}\right)=\frac{1}{4} \sum_{i, j}\left\langle e_{j}, B e_{i}\right\rangle\left(c\left(e_{i}\right) c\left(e_{j}\right)-\widehat{c}\left(e_{i}\right) \widehat{c}\left(e_{j}\right)\right) .
$$

If $B \in \operatorname{End}(T X)$ is symmetric, then the action of $B$ on $\Lambda\left(T^{*} X\right)$ as a derivation is given by

$$
-\sum_{i, j}\left\langle B e_{j}, e_{i}\right\rangle w\left(e^{j}\right) i\left(e_{i}\right)=-\frac{1}{2} \sum_{i, j}\left\langle B e_{j}, e_{i}\right\rangle c\left(e_{i}\right) \widehat{c}\left(e_{j}\right)-\frac{1}{2} \sum_{i}\left\langle B e_{i}, e_{i}\right\rangle .
$$

Definition 3.4. We define a 1-form on $X$ with values in the self-adjoint endomorphisms of $F$ by

$$
\omega\left(F, h^{F}\right):=\left(h^{F}\right)^{-1}\left(\nabla^{F} h^{F}\right),
$$


and a unitary connection for $\left(F, h^{F}\right)$ by

$$
\nabla^{F, u}:=\nabla^{F}+\frac{1}{2} \omega\left(F, h^{F}\right) .
$$

Here we view $h^{F}$ as an isomorphism $F \rightarrow F^{*}$.

We denote by $\nabla^{\Lambda\left(T^{*} X\right)}$ the connection on $\Lambda\left(T^{*} X\right)$ induced by $\nabla^{T X}$ and by $\nabla^{T X \otimes F, u}=\nabla^{\Lambda\left(T^{*} X\right)} \otimes \mathrm{Id}+\mathrm{Id} \otimes \nabla^{F, u}$ the unitary connection on $\Lambda\left(T^{*} X\right) \otimes F$ induced by $\nabla^{\Lambda\left(T^{*} X\right)}$ and $\nabla^{F, u}$.

Let $E$ be a vector bundle on $X$ and let $\nabla^{E}$ be any connection on $E$. In the sequel, we will use the notation

$$
\Delta^{E}:=-\sum_{i=1}^{m}\left(\left(\nabla_{e_{i}}^{E}\right)^{2}-\nabla_{\nabla_{e_{i}}^{T X}}^{E} e_{i}\right) .
$$

If $E$ and $\nabla^{E}$ are Hermitian, then $\Delta^{E}=\nabla^{E *} \nabla^{E}$ is the usual Bochner Laplacian.

The following Lichnerowicz formula was proved in [BiZ1, Thm.4.13].

Theorem 3.5. With $\kappa$ the scalar curvature of $\left(T X, g^{T X}\right)$ we have

$$
\begin{aligned}
& D^{2}=\Delta^{T X \otimes F, u}+\frac{\kappa}{4}+\frac{1}{8} \sum_{i, j, k, l}\left\langle e_{k}, R^{T X}\left(e_{i}, e_{j}\right) e_{l}\right\rangle c\left(e_{i}\right) c\left(e_{j}\right) \widehat{c}\left(e_{k}\right) \widehat{c}\left(e_{l}\right) \\
&+\frac{1}{4} \sum_{i}\left(\omega\left(F, h^{F}\right)\left(e_{i}\right)\right)^{2}-\frac{1}{8} \sum_{i, j}\left(c\left(e_{i}\right) c\left(e_{j}\right)-\widehat{c}\left(e_{i}\right) \widehat{c}\left(e_{j}\right)\right)\left(\omega\left(F, h^{F}\right)\right)^{2}\left(e_{i}, e_{j}\right) \\
&-\frac{1}{2} \sum_{i, j} c\left(e_{i}\right) \widehat{c}\left(e_{j}\right)\left(\nabla_{e_{i}}^{T X \otimes F, u} \omega\left(F, h^{F}\right)\right)\left(e_{j}\right) .
\end{aligned}
$$

Let $\left\{e_{\alpha}\right\}_{\alpha=1}^{m-1}$ be an orthonormal frame for $T Y$, with $e_{m}=e_{\mathfrak{n}} \in N$ as in subsection 3.1. The following result is [BiZ1, Prop. 4.9].

Proposition 3.6. Among all monomials in the variables $c\left(e_{\alpha}\right)$ and $\widehat{c}\left(e_{\alpha}\right)$ (up to permutations) only $c\left(e_{1}\right) \widehat{c}\left(e_{1}\right) \cdots c\left(e_{m-1}\right) \widehat{c}\left(e_{m-1}\right)$ has a nonzero supertrace in $\operatorname{End}\left(\Lambda\left(T^{*} Y\right)\right)$. Moreover,

$$
\operatorname{Tr}_{s}\left[c\left(e_{1}\right) \widehat{c}\left(e_{1}\right) \cdots c\left(e_{m-1}\right) \widehat{c}\left(e_{m-1}\right)\right]=(-2)^{m-1} .
$$

In the vector space $T Y \oplus T Y$, we view $\left\{e_{\alpha}\right\}$ as an orthonormal frame for the first summand and $\left\{\widehat{e}_{\alpha}\right\}$ as one for the second. Likewise, $\left\{e^{\alpha}\right\}$ and $\left\{\widehat{e^{\alpha}}\right\}$ denote the corresponding dual frames of the first and second summand in $T^{*} Y \oplus T^{*} Y$. Now we use the rescaling introduced by Bismut-Zhang [BiZ1, (4.20)] along the boundary $Y$ : For $1 \leq \alpha \leq m-1$ and $t>0$, set

$$
c_{t}\left(e_{\alpha}\right)=t^{-1 / 4} w\left(e^{\alpha}\right)-t^{1 / 4} i\left(e_{\alpha}\right), \quad \widehat{c}_{t}\left(\widehat{e_{\alpha}}\right)=t^{-1 / 4} w\left(\widehat{e^{\alpha}}\right)+t^{1 / 4} i\left(\widehat{e}_{\alpha}\right) .
$$

Since these operators satisfy the relations (3.22), we see that there is an algebra homomorphism, $\left.\psi_{t}: \operatorname{End}\left(\Lambda\left(T^{*} Y\right)\right) \rightarrow \operatorname{End}\left(\Lambda\left(T^{*} Y\right) \widehat{\otimes} \widehat{\Lambda\left(T^{*} Y\right.}\right)\right)$, mapping $c(e)$ to $c_{t}(e)$ and $\widehat{c}(e)$ to $\widehat{c}_{t}(\widehat{e})$ for $e \in T Y$. 
Since the operators $w\left(e^{I}\right) w\left(\widehat{e^{J}}\right) i\left(e_{K}\right) i\left(\widehat{e_{L}}\right)(I, J, K, L \subset\{1, \ldots, m-1\})$ are linearly independent in $\left.\operatorname{End}\left(\Lambda\left(T^{*} Y\right) \widehat{\otimes} \widehat{\Lambda\left(T^{*} Y\right.}\right)\right), \psi_{t}(B)$ is a linear combination of such operators, for any $B \in \operatorname{End}\left(\Lambda\left(T^{*} Y\right)\right)$. Let $\{C\}^{\max } \in \mathbb{R}$ be the coefficient of the monomial $w\left(e^{\{1, \ldots, m-1\}}\right) w\left(e^{\{1, \ldots, m-1\}}\right)$ in the expansion of $\left.C \in \operatorname{End}\left(\Lambda\left(T^{*} Y\right) \widehat{\otimes} \widehat{\Lambda\left(T^{*} Y\right.}\right)\right)$; then by Proposition 3.6, we have

$$
\operatorname{Tr}_{s}[B]=2^{m-1}(-1)^{\frac{m(m-1)}{2}} t^{\frac{m-1}{2}}\left\{\psi_{t}(B)\right\}^{\max } .
$$

3.4 Reformulation of the boundary condition. Now we reformulate the boundary condition (3.5). We use the notation in subsection 1.2. Let $\left\{e_{\alpha}\right\}_{\alpha=1}^{m-1}$ be an orthonormal frame of $\left(T Y, g^{T Y}\right)$. We denote by $\nabla^{T X \otimes F}$ the connection on $\Lambda\left(T^{*} X\right) \otimes F$ induced by $\nabla^{T X}$ and $\nabla^{F}$. Likewise, we introduce the corresponding objects induced by ${ }^{s p} \nabla^{T X}$ which are distinguished by a prefix $s p$. Especially, ${ }^{s p} \nabla^{\Lambda\left(T^{*} X\right)},{ }^{s p} \nabla^{T X \otimes F}$ are the connections on $\Lambda\left(T^{*} X\right), \Lambda\left(T^{*} X\right) \otimes F$ induced by ${ }^{s p} \nabla^{T X}$ and $\nabla^{F}$.

On $Y \times[0, \varepsilon) \subset X$ as in subsection 1.2, let $\left\{e_{\alpha}\right\}$ be an orthonormal basis of ${ }^{1} T X$. Let $A^{T Y} \in{ }^{2} T^{*} X \otimes \operatorname{End}\left({ }^{1} T X\right) \subset T^{*} X \otimes \operatorname{End}(T X)$ be defined by

$$
A^{T Y}\left(e_{m}\right) e_{\beta}=\sum_{\alpha=1}^{m-1}\left\langle e_{m}, A\left(e_{\alpha}\right) e_{\beta}\right\rangle e_{\alpha}, \quad A^{T Y}\left(e_{\alpha}\right)=0 .
$$

By (3.24), the action on $\Lambda\left(T^{*} X\right)$ induced by $A^{T Y}$ is defined by

$$
\begin{aligned}
& A^{\Lambda\left(T^{*} Y\right)}\left(e_{m}\right):=-\sum_{1 \leq \alpha, \beta \leq m-1}\left\langle e_{m}, A\left(e_{\alpha}\right) e_{\beta}\right\rangle w\left(e^{\alpha}\right) i\left(e_{\beta}\right) \\
& =-\frac{1}{2} \sum_{\alpha, \beta=1}^{m-1}\left\langle e_{m}, A\left(e_{\alpha}\right) e_{\beta}\right\rangle c\left(e_{\alpha}\right) \widehat{c}\left(e_{\beta}\right)-\frac{1}{2} \sum_{\alpha=1}^{m-1}\left\langle e_{m}, A\left(e_{\alpha}\right) e_{\alpha}\right\rangle, \\
& A^{\Lambda\left(T^{*} Y\right)}\left(e_{\alpha}\right):=0 .
\end{aligned}
$$

Proposition 3.7. The boundary condition (3.5) is equivalent to

$$
\left.i\left(e_{m}\right) \omega\right|_{Y}=\left.\left({ }^{s p} \nabla_{e_{m}}^{T X \otimes F}+A^{\Lambda\left(T^{*} Y\right)}\left(e_{m}\right)\right) w\left(e^{m}\right) \omega\right|_{Y}=0 .
$$

Proof. Since $\nabla^{T X}$ is torsion free, it is clear that (cf. [BGV, Prop. 1.22])

$$
d^{F}=\sum_{i=1}^{m} w\left(e^{i}\right) \nabla_{e_{i}}^{T X \otimes F} .
$$

By (1.8), (1.9), (3.23), and (3.35), we have on $Y$,

$$
i\left(e_{m}\right) d^{F}=i\left(e_{m}\right) \sum_{i=1}^{m} w\left(e^{i}\right)\left({ }^{s p} \nabla_{e_{i}}^{T X \otimes F}+\sum_{j, k=1}^{m}\left\langle e_{k}, A\left(e_{i}\right) e_{j}\right\rangle w\left(e^{k}\right) i\left(e_{j}\right)\right)
$$




$$
\begin{aligned}
& =i\left(e_{m}\right) \sum_{\alpha=1}^{m-1} w\left(e^{\alpha}\right)^{s p} \nabla_{e_{\alpha}}^{T X \otimes F} \\
& \quad+i\left(e_{m}\right) w\left(e^{m}\right)\left({ }^{s p} \nabla_{e_{m}}^{T X \otimes F}-\sum_{\alpha, \beta=1}^{m-1}\left\langle e_{m}, A\left(e_{\alpha}\right) e_{\beta}\right\rangle w\left(e^{\alpha}\right) i\left(e_{\beta}\right)\right) .
\end{aligned}
$$

Applying (3.36) to the kernel of $i\left(e_{m}\right)$, we get (3.34).

Let ${ }^{s p} \nabla^{T X, A}$ be the connection on $T X$ defined by

$$
{ }^{s p} \nabla^{T X, A}={ }^{s p} \nabla^{T X}+A^{T Y} .
$$

Let ${ }^{s p} R^{T X, A}$ be the curvature of ${ }^{s p} \nabla^{T X, A}$. Let ${ }^{s p} \nabla^{T X \otimes F, A}$ be the connection on $\Lambda\left(T^{*} X\right) \otimes F$ induced by ${ }^{s p} \nabla^{T X, A}$ and $\nabla^{F}$, then

$$
{ }^{s p} \nabla^{T X \otimes F, A}={ }^{s p} \nabla^{T X \otimes F}+A^{\Lambda\left(T^{*} Y\right)} .
$$

For $x=\left(y, x_{m}\right) \in \mathcal{U}_{\varepsilon}$, we identify $\left(\Lambda\left(T^{*} X\right) \otimes F\right)_{x}$ with $\left(\Lambda\left(T^{*} X\right) \otimes F\right)_{y}$ by parallel transport with respect to the connection ${ }^{s p} \nabla^{T X \otimes F, A}$ along the curve $[0,1] \ni u \mapsto\left(y, u x_{m}\right)$ which induces an identification

$$
C^{\infty}\left(\mathcal{U}_{\varepsilon}, \Lambda\left(T^{*} X\right) \otimes F\right) \rightarrow C^{\infty}\left([-\epsilon, \epsilon], C^{\infty}\left(Y,\left.\left(\Lambda\left(T^{*} X\right) \otimes F\right)\right|_{Y}\right)\right) .
$$

3.5 Rescaling. To calculate the limit of $I_{t}(y)$ in (3.11) when $t \rightarrow 0$, we will localize the problem on $Y$. We construct first a trivialization, (which may be viewed as a simpler version of what has been used in [BiL, $\S 13 \mathrm{~d}), \mathrm{e})]$, where $Y$ is a sub-manifold of the complex manifold $X$ ); then by using (3.31), we transfer our problem for operators acting on $C^{\infty}\left(\mathbb{R}_{+}^{m}, \Lambda\left(T^{*} X\right) \otimes F\right)$, where $\mathbb{R}_{+}^{m}=\mathbb{R}^{m-1} \times \mathbb{R}_{+}$, to a problem for operators acting on

$$
\left.C^{\infty}\left(\mathbb{R}_{+}^{m},\left(\Lambda\left(T^{*} Y\right) \widehat{\otimes} \widehat{\Lambda\left(T^{*} Y\right.}\right) \widehat{\otimes} \Lambda\left(N^{*}\right) \otimes F\right)_{y_{0}}\right),
$$

by an appropriate rescaling.

Denote by $r(W)$ the injectivity radius of the Riemannian manifold $\left(W, g^{T W}\right)$, we choose $\epsilon$ with $0<\epsilon \leq \frac{1}{4} \min \{r(X), r(Y)\}$. If $y \in Y$ and $Z \in T_{y} Y$, then $\mathbb{R} \ni u \mapsto \gamma(u)=\exp _{y}^{Y}(u Z) \in Y$ denotes the geodesic along $Y$ such that $\gamma(0)=x, \dot{\gamma}(0)=Z$. Also, for any metric space $\tilde{X}$, we denote by $B_{\varepsilon}^{\widetilde{X}}(x)$ the metric ball of radius $\varepsilon>0$ around $x \in \tilde{X}$. Then $T_{y} Y \ni Z^{\prime} \rightarrow \exp _{y}^{Y}\left(Z^{\prime}\right)$ identifies $B_{\varepsilon}^{T_{y} Y}(0)$ to $B_{\varepsilon}^{Y}(y)$; and $\Psi^{\prime}:\left(T_{y} Y \oplus N_{y}\right) \ni$ $\left(Z^{\prime}, z_{m} e_{\mathfrak{n}}\right) \rightarrow\left(\exp _{y}^{Y}\left(Z^{\prime}\right), z_{m}\right)$ identifies $B_{\varepsilon}^{T_{y} Y}(0) \times B_{\varepsilon}^{N_{y}}(0)$ to $B_{\varepsilon}^{Y}(y) \times[-\epsilon, \epsilon]$. We will always use these identifications in the following.

Now we fix $y_{0} \in Y$. For $Z^{\prime} \in B_{\varepsilon}^{T_{y_{0}} Y}(0)$, we identify $\left(\Lambda\left(T^{*} X\right) \otimes F\right)_{Z^{\prime}}$ with $\left(\Lambda\left(T^{*} X\right) \otimes F\right)_{y_{0}}$ by parallel transport with respect to the connection ${ }^{s p} \nabla^{T X \otimes F, A}$ along the curve $[0,1] \ni u \mapsto u Z^{\prime}$. In fact, the above two steps identify $\left(\Lambda\left(T^{*} X\right) \otimes F\right)_{Z}\left(Z=\left(Z^{\prime}, Z_{m}\right) \in B_{\varepsilon}^{T_{y_{0}} Y}(0) \times B_{\varepsilon}^{N_{y_{0}}}(0)\right)$ 
with $\left(\Lambda\left(T^{*} X\right) \otimes F\right)_{y_{0}}$, by parallel transport with respect to the connection ${ }^{s p} \nabla^{T X \otimes F, A}$ along the curve $\gamma_{Z}:[0,1] \ni u \mapsto u Z^{\prime},[1,2] \ni u \mapsto$ $Z^{\prime}+(u-1) Z_{m}$.

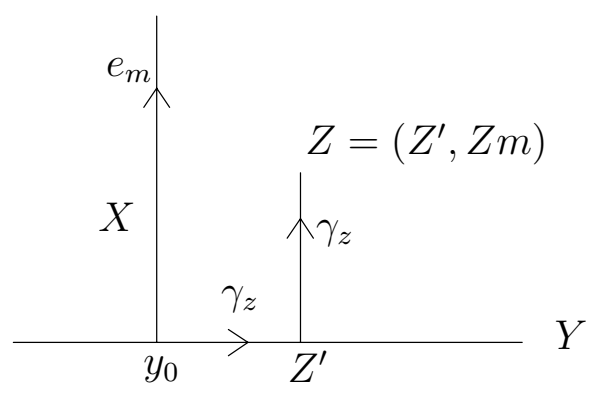

This defines a trivialization $\Psi:\left(B_{\epsilon}^{Y}\left(y_{0}\right) \times[-\epsilon, \epsilon]\right) \times\left(\Lambda\left(T^{*} X\right) \otimes F\right)_{y_{0}} \rightarrow$ $\left.\left(\Lambda\left(T^{*} X\right) \otimes F\right)\right|_{B_{\epsilon}^{Y}\left(y_{0}\right) \times[-\epsilon, \epsilon]}$. Thus we obtain an invertible linear map

$\Phi: C^{\infty}\left(B_{\epsilon}^{Y}\left(y_{0}\right) \times[-\epsilon, \epsilon], \Lambda\left(T^{*} X\right) \otimes F\right)$

$$
\rightarrow C^{\infty}\left(B_{\epsilon}^{T_{y} Y}(0) \times[-\epsilon, \epsilon],\left(\Lambda\left(T^{*} X\right) \otimes F\right)_{y_{0}}\right),
$$

$(\Phi \sigma)\left(Z^{\prime}, Z_{m}\right)=\Psi^{-1}\left(\sigma\left(\Psi^{\prime}\left(Z^{\prime}, Z_{m}\right)\right)\right)$

For an operator $C$ on $C^{\infty}\left(B_{\epsilon}^{Y}\left(y_{0}\right) \times[-\epsilon, \epsilon], \Lambda\left(T^{*} X\right) \otimes F\right)$ we write $C_{\Phi}:=$ $\Phi C \Phi^{-1}$.

We use the notation in subsection 1.2. Let $\left\{e_{\alpha}\right\}_{\alpha=1}^{m-1}$ be an orthonormal frame of $T_{y_{0}} Y$. Denote by $\widetilde{e}_{\alpha}(Z)$ the parallel transport of $e_{\alpha}$ with respect to ${ }^{s p} \nabla^{T X}$ along $\gamma_{Z}$, then $\left\{\widetilde{e}_{\alpha}(Z)\right\}_{\alpha=1}^{m-1}$ is an orthonormal basis of ${ }^{1} T X_{Z}$, while $\widetilde{e}_{m}=e_{m}=\partial / \partial x_{m}$ spans ${ }^{2} T X_{Z}$.

By Lemma 3.3, we know that our calculation will localize near $Y$. In order to use rescaling arguments, we want to view $D_{a}^{2}$ in the chosen trivialization as the restriction of a suitable differential operator defined on $\mathbb{R}^{m}=T_{y_{0}} Y \oplus N_{y_{0}}$, with general point $z=\left(z^{\prime}, z_{m}\right)$ corresponding to $Z=\left(Z^{\prime}, Z_{m}\right)$ via the basis $\left\{e_{i}\right\}$. To construct the extension explicitly, let $\varphi: \mathbb{R} \rightarrow[0,1]$ be a smooth function such that

$$
\varphi(u)=\left\{\begin{array}{lll}
1 & \text { for } \quad|u| \leq \epsilon \\
0 & \text { for } \quad|u| \geq 2 \epsilon .
\end{array}\right.
$$

Denote by $\nabla:=\nabla^{T_{y_{0}} X}$ the canonical connection on $T_{y_{0}} X$ such that $\nabla_{U}$ is the ordinary differentiation operator on $T_{y_{0}} X$ in the direction $U$, and let $\Delta^{T_{y_{0}} X}=-\sum_{i} \partial^{2} / \partial z_{i}^{2}$ be the standard Euclidean Laplacian on $T_{y_{0}} X$ with respect to the metric $g^{T_{y_{0}} X}$. We introduce the operator

$$
L_{t}^{(1)}:=\varphi^{2}(|z|) t\left(D^{2}\right)_{\Phi}+\left(1-\varphi^{2}(|z|)\right)\left(t \Delta^{T_{y_{0}} X}\right),
$$


on $C^{\infty}\left(\mathbb{R}^{m},\left(\Lambda\left(T^{*} X\right) \otimes F\right)_{y_{0}}\right)$ and we denote by $L_{t, a}^{(1)}$ its restriction to elements of $C^{\infty}\left(\mathbb{R}_{+}^{m},\left(\Lambda\left(T^{*} X\right) \otimes F\right)_{y_{0}}\right)$ satisfying

$$
\left\{\begin{aligned}
\left\{\varphi^{2}(|z|)\left(i\left(e_{m}(z)\right)\right)_{\Phi}\right. & \left.+\left(1-\varphi^{2}(|z|)\right) i\left(e_{m}\right)\right\}\left.\omega\right|_{z_{m}=0}=0 \\
\left\{\varphi ^ { 2 } ( | z | ) \left(w\left(e^{m}(z)\right)^{s p} \nabla_{e_{m}}^{T X} \otimes F, A\right.\right. & )_{\Phi} \\
& \left.+\left(1-\varphi^{2}(|z|)\right) w\left(e^{m}\right) \nabla_{e_{m}}\right\}\left.\omega\right|_{z_{m}=0}=0 .
\end{aligned}\right.
$$

Note that if $U, V$ are smooth sections of $T X$ then, for $\mathbf{c}=c$ or $\widehat{c}$,

$$
\left[{ }^{s p} \nabla_{U}^{\Lambda\left(T^{*} X\right)}, \mathbf{c}(V)\right]=\mathbf{c}\left({ }^{s p} \nabla_{U}^{T X} V\right) .
$$

From (1.9), (3.38), and (3.42), we derive the identities for $z \in \mathbb{R}^{m}$,

$$
\left(\mathbf{c}\left(e_{m}\right)\right)_{\Phi}(z)=\mathbf{c}\left(e_{m}\right), \quad\left({ }^{s p} \nabla_{e_{m}}^{T X \otimes F, A}\right)_{\Phi, z}=\nabla_{e_{m}} .
$$

Then, by (3.43), the boundary condition (3.41) is equivalent to

$$
\left\{\begin{array}{l}
\left.i\left(e_{m}\right) \omega\right|_{z_{m}=0}=0 \\
\left.\nabla_{e_{m}} w\left(e^{m}\right) \omega\right|_{z_{m}=0}=0 .
\end{array}\right.
$$

For $t>0, \sigma \in C^{\infty}\left(\mathbb{R}^{m},\left(\Lambda\left(T^{*} X\right) \otimes F\right)_{y_{0}}\right)$, and $z \in \mathbb{R}^{m}$, set

$$
\left(S_{t} \sigma\right)(z):=\sigma(z / \sqrt{t}), \quad L_{t}^{(2)}:=S_{t}^{-1} L_{t}^{(1)} S_{t}
$$

such that $L_{t}^{(2)}$ is a differential operator on $\mathbb{R}^{m}$ with coefficients in $\operatorname{End}\left(\Lambda\left(T^{*} X\right) \otimes F\right)_{y_{0}}$. Next we enlarge the coefficient space (as is common in local index theory) and replace $\left(\Lambda\left(T^{*} X\right) \otimes F\right)_{y_{0}}$ by

$$
\left.K_{y_{0}}=\left(\Lambda\left(T^{*} Y\right) \widehat{\otimes} \widehat{\Lambda\left(T^{*} Y\right.}\right) \widehat{\otimes} \Lambda\left(N^{*}\right) \otimes F\right)_{y_{0}} .
$$

Using (3.21), (3.30), and the algebra homomorphism $\psi_{t}$, we see that $L_{t}^{(2)}$ induces a differential operator $L_{t}^{(3)}$ with coefficients in $\operatorname{End}\left(K_{y_{0}}\right)$ which has the Clifford variables $c\left(e_{\alpha}\right)$ and $\widehat{c}\left(e_{\alpha}\right)$ replaced by $c_{t}\left(e_{\alpha}\right)$ and $\widehat{c_{t}}\left(\widehat{e_{\alpha}}\right)$ $(1 \leq \alpha \leq m-1)$.

If $C$ is any suitable differential operator, we will denote by $C_{t}^{(3)}$ the operator obtained from $C$ by rescaling the Clifford variables and the variables $z$, as prescribed in (3.30), (3.45).

We will denote by $L_{t, a}^{(i)}$ the operator $L_{t}^{(i)}$ with the corresponding boundary condition (3.44). Now denote by $e^{-L_{t, a}^{(i)}}(z, w)\left(z, w \in \mathbb{R}_{+}^{m}, i=1,2,3\right)$ the smooth kernels associated to $e^{-L_{t, a}^{(i)}}$ with the corresponding boundary condition, calculated with respect to $d v_{T_{y_{0}} Y}\left(w^{\prime}\right) d v_{N}\left(w_{m}\right)$, and likewise let $e^{-L_{t}^{(i)}}(z, w)\left(z, w \in \mathbb{R}^{m}\right)$ be the kernel associated to $e^{-L_{t}^{(i)}}$. For $z, w \in \mathbb{R}_{+}^{m}$, set

$$
P_{t}^{(i)}(z, w):=\left(e^{-L_{t, a}^{(i)}}-e^{-L_{t}^{(i)}}\right)(z, w) .
$$

By (3.11) and the argument of the finite propagation speed of the wave equation as in Lemma 3.3, we know that there exist $C, c>0$ such that for 
$0 \leq z_{m}<\epsilon / 2,0<t<1$

$$
\left|P_{t}\left(\left(0, z_{m}\right),\left(0, z_{m}\right)\right) \kappa\left(0, z_{m}\right)-P_{t}^{(1)}\left(\left(0, z_{m}\right),\left(0, z_{m}\right)\right)\right| \leq C e^{-c / t} .
$$

By $(3.45)$, for $z, w \in \mathbb{R}_{+}^{m}$,

$$
P_{t}^{(1)}(z, w)=t^{-m / 2} P_{t}^{(2)}\left(\frac{z}{\sqrt{t}}, \frac{w}{\sqrt{t}}\right),
$$

and by (3.31),

$$
\begin{aligned}
\operatorname{Tr}_{s}\left[P_{t}^{(2)}\right. & \left.\left(\left(0, z_{m}\right),\left(0, z_{m}\right)\right)\right] \\
& =2^{m-1}(-1)^{\frac{m(m-1)}{2}} t^{(m-1) / 2} \operatorname{Tr}_{s}\left[\left[P_{t}^{(3)}\left(\left(0, z_{m}\right),\left(0, z_{m}\right)\right)\right]^{\max }\right] .
\end{aligned}
$$

Thus we derive the identity

$$
\begin{aligned}
& \int_{0}^{t^{1 / 4}} \operatorname{Tr}_{s}\left[P_{t}^{(1)}\left(\left(0, z_{m}\right),\left(0, z_{m}\right)\right)\right] d v_{N}\left(z_{m}\right) \\
= & \int_{0}^{t^{1 / 4}} 2^{m-1}(-1)^{\frac{m(m-1)}{2}} t^{-\frac{1}{2}} \operatorname{Tr}_{s}\left[\left[P_{t}^{(3)}\left(\left(0, \frac{z_{m}}{\sqrt{t}}\right),\left(0, \frac{z_{m}}{\sqrt{t}}\right)\right)\right]^{\max }\right] d v_{N}\left(z_{m}\right) \\
= & \int_{0}^{t^{-1 / 4}} 2^{m-1}(-1)^{\frac{m(m-1)}{2}} \operatorname{Tr}_{s}\left[\left[P_{t}^{(3)}\left(\left(0, z_{m}\right),\left(0, z_{m}\right)\right)\right]^{\max }\right] d v_{N}\left(z_{m}\right) .
\end{aligned}
$$

Hence to calculate the limit (3.11), we only need to calculate the limit (3.51) when $t \rightarrow 0$, by (3.48). Calculating the limit of the operator $L_{t, a}^{(3)}$ we arrive at the following model problem.

Theorem 3.8. The above limit equation is

$$
\left\{\begin{array}{l}
\left.i\left(e_{m}\right) \omega\right|_{z_{m}=0}=0, \\
\left.\nabla_{e_{m}} w\left(e^{m}\right) \omega\right|_{z_{m}=0}=0, \\
\left(\frac{\partial}{\partial t}-\left(\frac{\partial}{\partial z_{m}}-\dot{S}\left(y_{0}\right)\right)^{2}-\sum_{\alpha=1}^{m-1} \frac{\partial^{2}}{\partial z_{\alpha}^{2}}+\frac{1}{2}\left(\dot{R}^{T X} \mid Y\right)_{y_{0}}\right) \omega=0 .
\end{array}\right.
$$

Proof. Let ${ }^{s p} \Gamma^{T X, A}, \Gamma^{F}$ and $\Gamma^{F, u}$ be the respective connection forms of ${ }^{s p} \nabla^{T X, A}, \nabla^{F}$ and $\nabla^{F, u}$, computed with respect to the frame $\left\{\widetilde{e}_{i}\right\}$ for $T X$ and any fixed frame for $F$ which is parallel with respect to $\nabla^{F}$ along the curves $\gamma_{Z}$ (cf. the beginning of subsection 3.5). By (1.8), (3.37) we know that for $Z \in T_{y_{0}} X$,

$$
\begin{gathered}
\nabla^{T X}={ }^{s p} \nabla^{T X, A}+A_{Z}-A_{Z}^{T Y}, \\
\Gamma^{F, u}=\Gamma^{F}+\frac{1}{2} \omega\left(F, h^{F}\right), \quad \Gamma^{F}=0 .
\end{gathered}
$$

Thus from (3.23), (3.38), (3.43), and (3.53),

$$
\begin{aligned}
\nabla^{T X \otimes F, u} & ={ }^{s p} \nabla^{T X \otimes F, A}-A^{\Lambda\left(T^{*} Y\right)}(\cdot) \\
& +\frac{1}{2} \sum_{\alpha=1}^{m-1}\left\langle A(\cdot) e_{m}, \widetilde{e}_{\alpha}\right\rangle\left(c\left(e_{m}\right) c\left(\widetilde{e}_{\alpha}\right)-\widehat{c}\left(e_{m}\right) \widehat{c}\left(\widetilde{e}_{\alpha}\right)\right)+\Gamma^{F, u}(\cdot) .
\end{aligned}
$$


From (3.23), (3.24), we know also

$$
\begin{gathered}
{ }^{s p} \nabla^{T X \otimes F, A}=\nabla \cdot+\sum_{i, j=1}^{m}\left\langle{ }^{s p} \Gamma_{Z}^{T X, A}(\cdot) e_{i}, e_{j}\right\rangle w\left(e^{j}\right) i\left(e_{i}\right) \\
=\nabla \cdot+\frac{1}{4} \sum_{\alpha, \beta=1, \alpha \neq \beta}^{m-1}\left\langle{ }^{s p} \Gamma_{Z}^{T X, A}(\cdot) \widetilde{e}_{\alpha}, \widetilde{e}_{\beta}\right\rangle\left(c\left(\widetilde{e}_{\alpha}\right) c\left(\widetilde{e}_{\beta}\right)-\widehat{c}\left(\widetilde{e}_{\alpha}\right) \widehat{c}\left(\widetilde{e}_{\beta}\right)\right) \\
-\frac{1}{4} \sum_{\alpha, \beta=1}^{m-1}\left\langle{ }^{s p} \Gamma_{Z}^{T X, A}(\cdot) \widetilde{e}_{\alpha}, \widetilde{e}_{\beta}\right\rangle\left(c\left(\widetilde{e}_{\alpha}\right) \widehat{c}\left(\widetilde{e}_{\beta}\right)+c\left(\widetilde{e}_{\beta}\right) \widehat{c}\left(\widetilde{e}_{\alpha}\right)\right)-\frac{1}{2} \sum_{\alpha=1}^{m-1}\left\langle{ }^{s p} \Gamma_{Z}^{T X, A}(\cdot) \widetilde{e}_{\alpha}, \widetilde{e}_{\alpha}\right\rangle .
\end{gathered}
$$

From [ABP, Prop. 3.7] (cf. [BGV, Thm. 1.18]) and (3.32) we infer that ${ }^{s p} \Gamma_{Z}^{T X, A}(U)=\frac{1}{2}^{s p} R_{y_{0}}^{T X}(Z, U)+\mathcal{O}\left(|Z|^{2}\right) U, \quad$ if $Z, U \in T_{y_{0}} Y$.

By construction,

$$
{ }^{s p} \Gamma_{Z}^{T X, A}(U)=0 \quad \text { if } Z \in T_{y_{0}} X, U \in N_{y_{0}} .
$$

Observing that by $(3.56),(3.57)$ and $\left[e_{m}, e_{\alpha}\right]=0$, for $X$ a section of $T X$ such that ${ }^{s p} \nabla_{e_{m}}^{T X, A} X=0$, we have

$$
{ }^{s p} R_{y_{0}}^{T X, A}\left(e_{m}, e_{\alpha}\right) X={ }^{s p} \nabla_{e_{m}}^{T X}{ }^{s p} \nabla_{e_{\alpha}}^{T X, A} X=\left(\nabla_{e_{m}}{ }^{s p} \Gamma^{T X, A}\left(e_{\alpha}\right)\right)_{y_{0}} X .
$$

Thus we find that

$$
{ }^{s p} \Gamma_{Z}^{T X, A}(U)={ }^{s p} R_{y_{0}}^{T X, A}(Z, U)+\mathcal{O}\left(|Z|^{2}\right) U \quad \text { if } Z \in N_{y_{0}}, U \in T_{y_{0}} Y .
$$

Note that the equations (3.56), (3.57), and (3.59) were obtained in [BiL, (13.64)-(13.66)].

From the definition of $\widetilde{e}_{\alpha}$ and (3.42), we get for $z^{\prime} \in \mathbb{R}^{m-1}, \mathbf{c}=c$ or $\widehat{c}$,

$$
\left(\mathbf{c}\left(\widetilde{e}_{\alpha}\right)\right)_{\Phi}\left(z^{\prime}\right)=\mathbf{c}\left(e_{\alpha}\right) \text {. }
$$

From (3.33), (3.38), and (3.42), we have for $V \in{ }^{1} T X$

$$
\begin{aligned}
& {\left[{ }^{s p} \nabla_{e_{m}}^{T X \otimes F, A}, c(V)\right]=c\left({ }^{s p} \nabla_{e_{m}}^{T X} V\right)-\sum_{\beta=1}^{m-1}\left\langle e_{m}, A(V) \widetilde{e}_{\beta}\right\rangle \widehat{c}\left(\widetilde{e}_{\beta}\right),} \\
& \left.{ }^{s p} \nabla_{e_{m}}^{T X \otimes F, A}, \widehat{c}(V)\right]=\widehat{c}\left({ }^{s p} \nabla_{e_{m}}^{T X} V\right)-\sum_{\beta=1}^{m-1}\left\langle e_{m}, A\left(\widetilde{e}_{\beta}\right) V\right\rangle c\left(\widetilde{e}_{\beta}\right) .
\end{aligned}
$$

Thus, from (3.60), (3.61), we get for $z \in \mathbb{R}^{m}$

$$
\begin{aligned}
& \left(c\left(\widetilde{e}_{\alpha}\right)\right)_{\Phi}(z)=\left(1+\mathcal{O}\left(|z|^{2}\right)\right) c\left(e_{\alpha}\right)-\sum_{\beta=1}^{m-1}\left(z_{m}\left\langle e_{m}, A\left(e_{\alpha}\right) e_{\beta}\right\rangle+\mathcal{O}\left(|z|^{2}\right)\right) \widehat{c}\left(e_{\beta}\right), \\
& \left(\widehat{c}\left(\widetilde{e}_{\alpha}\right)\right)_{\Phi}(z)=\left(1+\mathcal{O}\left(|z|^{2}\right)\right) \widehat{c}\left(e_{\alpha}\right)-\sum_{\beta=1}^{m-1}\left(z_{m}\left\langle e_{m}, A\left(e_{\beta}\right) e_{\alpha}\right\rangle+\mathcal{O}\left(|z|^{2}\right)\right) c\left(e_{\beta}\right) .
\end{aligned}
$$


Now from Theorem 3.5, (1.15), (3.30), (3.33), (3.43), (3.54), (3.55), (3.56)-(3.59) and (3.62) we conclude that as $t \rightarrow 0$,

$$
L_{t}^{(3)} \rightarrow L_{0}^{(3)}:=-\left(\frac{\partial}{\partial z_{m}}-\dot{S}\left(y_{0}\right)\right)^{2}-\sum_{\alpha=1}^{m-1} \frac{\partial^{2}}{\partial z_{\alpha}^{2}}+\frac{1}{2}\left(\dot{R}^{T X} \mid Y\right)_{y_{0}}
$$

in the sense of distributions.

3.6 Uniform estimates for certain heat kernels. Let $e^{-u L_{t}^{(3)}}(z, w)$ be the smooth kernel of $e^{-u L_{t}^{(3)}}$ on $\mathbb{R}^{m}$ for $z, w \in \mathbb{R}^{m}$, calculated with respect to $d v_{T_{y_{0}} X}(w)$. Likewise, for $z, w \in \mathbb{R}_{+}^{m}$, let $e^{-u L_{t, a}^{(3)}}(z, w)$ be the smooth kernel of $e^{-u L_{t, a}^{(3)}}$, calculated with respect to $d v_{T_{y_{0}} X}(w)$.

The following two theorems arise as special cases of the main results in [Bi2, §11], [BiL, §11] if we set $Z_{0}=0, T=1, V=0$ (in the notation used there).

Theorem 3.9. For any $k \in \mathbb{N}$, there are constants $C, C^{\prime}>0$ and $r \in \mathbb{N}$ such that

$$
u^{\frac{m}{2}}\left|\frac{\partial^{|\alpha|+\left|\alpha^{\prime}\right|}}{\partial z^{\alpha} \partial w^{\alpha^{\prime}}} e^{-u L_{t}^{(3)}}(\sqrt{u} z, \sqrt{u} w)\right| \leq C^{\prime}(1+|z|+|w|)^{r} e^{-C|z-w|^{2}},
$$

uniformly for $|\alpha|,\left|\alpha^{\prime}\right| \leq k, u, t \in(0,1], z, w \in \mathbb{R}^{m}$.

Theorem 3.10. For $u>0$ fixed, we have

$$
\lim _{t \rightarrow 0} e^{-u L_{t}^{(3)}}(z, w)=e^{-u L_{0}^{(3)}}(z, w),
$$

uniformly on compact subsets of $\mathbb{R}^{m} \times \mathbb{R}^{m}$.

Sketch of the proof. Using standard methods of functional analysis one proves the estimate (3.64) without the exponential factor [BiL, Thm. 11.13]. To improve this to (3.64), one applies in addition the finite propagation speed for hyperbolic equations. In our case, each step is somewhat simpler than loc. cit. as our operator is simpler.

Now, as $L_{t}^{(3)} \rightarrow L_{0}^{(3)}$ for $t \rightarrow 0$ in the weak operator topology, we get

$$
\lim _{t \rightarrow 0} e^{-u L_{t}^{(3)}}=e^{-u L_{0}^{(3)}} .
$$

Thus, using the uniform bounds of (3.64), we get Theorem 3.10.

To establish the analogous estimates for $e^{-u L_{t, a}^{(3)}}$ and the uniform estimate (3.117) to be used later, we will first apply the techniques used in $[\mathrm{Bi} 2, \S 11]$ and [BiL, $\S 11]$ to our boundary value problem. Combining this with the finite propagation speed for boundary value problems $[\mathrm{T}, \S 6.1]$, we achieve the desired estimates. 
If $C$ is any differential operator on $C^{\infty}\left(\mathbb{R}^{m},\left(\Lambda\left(T^{*} X\right) \otimes F\right)_{y_{0}}\right)$, we denote by $C_{t, u}^{(4)}$ the operator obtained from $C$ by rescaling the coordinate $z$ by $S_{t u}$ in (3.45), and the Clifford variables as in (3.30).

Using (3.45), we set

$$
L_{t, u}^{(4)}:=S_{u}^{-1} u L_{t}^{(3)} S_{u} .
$$

We denote by $L_{t, u, a}^{(4)}$ the operator $L_{t, u}^{(4)}$ with domain defined by the boundary condition (3.44) (which is invariant under rescaling). We form the heat kernels for the operators $L_{t, u}^{(4)}$ and $L_{t, u, a}^{(4)}$ exactly as before. Then we get from $(3.67)$

$$
e^{-u L_{t, a}^{(3)}}(\sqrt{u} z, \sqrt{u} w)=u^{-m / 2} e^{-L_{t, u, a}^{(4)}}(z, w) .
$$

Now we equip $\Lambda\left(T^{*} X\right)_{y_{0}}$ with the metric induced by $g^{T_{y_{0}} X}$ and denote by $\mid$ the corresponding norm. We put

$$
\begin{gathered}
\left.K_{r, y_{0}}:=\oplus_{p+q=r}\left(\Lambda^{p}\left(T^{*} Y\right) \widehat{\otimes} \widehat{\Lambda^{q}\left(T^{*} Y\right.}\right) \widehat{\otimes} \Lambda\left(N^{*}\right) \otimes F\right)_{y_{0}}, \\
\left.K_{y_{0}}^{\prime}:=\left(\Lambda\left(T^{*} Y\right) \widehat{\otimes} \widehat{\Lambda\left(T^{*} Y\right.}\right) \otimes F\right)_{y_{0}} .
\end{gathered}
$$

As before, $\left\{e_{i}\right\}$ is an orthonormal basis of $T_{y_{0}} X$, and $\nabla_{Z}$ denotes the standard derivative on $T_{y_{0}} X$ in the direction $Z$. For $u, t \in(0,1], z \in \mathbb{R}^{m}$, set, with $\varphi$ from (3.39),

$$
g_{t, u}(z)=1+\left(1+u|z|^{2}\right)^{1 / 2} \varphi\left(\frac{\sqrt{t u}|z|}{2}\right) .
$$

Definition 3.11. For $k \in \mathbb{N}, t, u \in(0,1], \sigma \in C^{\infty}\left(\mathbb{R}_{+}^{m}, K_{r, y_{0}}\right)$, set

$$
\begin{aligned}
\|\sigma\|_{t, u, 0}^{2} & :=\int_{\mathbb{R}_{+}^{m}}|\sigma|^{2} g_{t, u}(z)^{2(2 m-2-r)} d v_{T_{y_{0}} X}(z), \\
\|\sigma\|_{t, u, k}^{2} & :=\sum_{l=0}^{k} \sum_{i_{1}, \ldots, i_{l}=1}^{m}\left\|\nabla_{e_{i_{1}}} \cdots \nabla_{e_{i_{l}}} \sigma\right\|_{t, u, 0}^{2} .
\end{aligned}
$$

We denote by $\langle\cdot, \cdot\rangle_{t, u, 0}$ the corresponding inner product. We also denote by ||$_{L^{2}}$ the the norm of ordinary Hermitian product on $L^{2}\left(\mathbb{R}_{+}^{m}, K_{y_{0}}\right)$.

Let $C_{0, t, u, a}^{\infty}\left(\mathbb{R}_{+}^{m}, K_{y_{0}}\right)$ be the elements of $C^{\infty}\left(\mathbb{R}_{+}^{m}, K_{y_{0}}\right)$ with compact support which verify the boundary condition (3.44). Let $H_{t, u, a}^{k}$ be the Hilbert space completion of $C_{0, t, u, a}^{\infty}\left(\mathbb{R}_{+}^{m}, K_{y_{0}}\right)$ with respect to the norm (3.71b), such that $H_{t, u, a}^{0}=L^{2}\left(\mathbb{R}_{+}^{m}, K_{y_{0}}\right)$ as vector spaces. Let $H_{t, u, a}^{-1}$ be the Sobolev space of order -1 associated with $C_{0, t, u, a}^{\infty}\left(\mathbb{R}_{+}^{m}, K_{y_{0}}\right)$ and let \|\|$_{t, u,-1}$ be the norm on $H_{t, u, a}^{-1}$ defined by $\|\sigma\|_{t, u,-1}=\sup _{0 \neq \sigma^{\prime} \in H_{t, u, a}^{1}}\left|\left\langle\sigma^{\prime}, \sigma\right\rangle_{t, u, 0}\right| /\left\|\sigma^{\prime}\right\|_{t, u, 1}$.

The following theorem is analogous to [BiL, Thm. 11.26]. 
Theorem 3.12. There exist constants $C_{1}, C_{2}, C_{3}>0$ such that for $u, t \in(0,1]$ and any $\sigma, \sigma^{\prime} \in C_{0, t, u, a}^{\infty}\left(\mathbb{R}_{+}^{m}, K_{y_{0}}\right)$

$$
\begin{gathered}
\operatorname{Re}\left\langle L_{t, u, a}^{(4)} \sigma, \sigma\right\rangle_{t, u, 0} \geq C_{1}\|\sigma\|_{t, u, 1}^{2}-C_{2}\|\sigma\|_{t, u, 0}^{2}, \\
\left|\operatorname{Im}\left\langle L_{t, u, a}^{(4)} \sigma, \sigma\right\rangle_{t, u, 0}\right| \leq C_{3}\|\sigma\|_{t, u, 1}\|\sigma\|_{t, u, 0}, \\
\left|\left\langle L_{t, u, a}^{(4)} \sigma, \sigma^{\prime}\right\rangle_{t, u, 0}\right| \leq C_{3}\|\sigma\|_{t, u, 1}\left\|\sigma^{\prime}\right\|_{t, u, 1} .
\end{gathered}
$$

Proof. Let $\Gamma^{T X \otimes F, u}$ be the connection form of the connection $\nabla^{T X \otimes F, u}$ on $\Lambda\left(T^{*} X\right) \otimes F$ associated with the frame induced from the trivialization $\Psi$. By (3.28), we get for $z \in \mathbb{R}^{m}$

$$
\begin{gathered}
L_{t, u}^{(4)}(z)=\left(1-\varphi^{2}(\sqrt{t u}|z|)\right) \Delta^{T_{y_{0}} X} \\
+\varphi^{2}(\sqrt{t u}|z|)\left[-\sum_{i=1}^{m}\left(\nabla_{\widetilde{e}_{i}(\sqrt{t u} z)}+\sqrt{t u}\left\{\Gamma_{z}^{T X \otimes F, u}\left(\widetilde{e}_{i}(z)\right)\right\}_{t, u}^{(4)}\right)^{2}\right. \\
+\sqrt{t u} \sum_{i=1}^{m}\left(\nabla_{\nabla_{\widetilde{e}_{i}}^{T} X \widetilde{e}_{i}(\sqrt{t u} z)}+\sqrt{t u}\left\{\Gamma_{z}^{T X \otimes F, u}\left(\nabla_{\widetilde{e}_{i}}^{T X} \widetilde{e}_{i}\right)\right\}_{t, u}^{(4)}\right)+\frac{t u \kappa}{4}(\sqrt{t u} z) \\
+\left\{\frac{t u}{8}\left\langle\widetilde{e}_{k}, R^{T X}\left(\widetilde{e}_{i}, \widetilde{e}_{j}\right) \widetilde{e}_{l}\right\rangle c\left(\widetilde{e}_{i}\right) c\left(\widetilde{e}_{j}\right) \widehat{c}\left(\widetilde{e}_{k}\right) \widehat{c}\left(\widetilde{e}_{l}\right)\right\}_{t, u}^{(4)}(z) \\
+t u\left\{\frac{1}{4}\left(\omega\left(F, h^{F}\right)\left(\widetilde{e}_{i}\right)\right)^{2}-\frac{1}{8}\left(c\left(\widetilde{e}_{i}\right) c\left(\widetilde{e}_{j}\right)-\widehat{c}\left(\widetilde{e}_{i}\right) \widehat{c}\left(\widetilde{e}_{j}\right)\right)\left(\omega\left(F, h^{F}\right)\right)^{2}\left(\widetilde{e}_{i}, \widetilde{e}_{j}\right)\right\}_{t, u}^{(4)}(z) \\
\left.-\frac{1}{2} t u\left\{c\left(\widetilde{e}_{i}\right) \widehat{c}\left(\widetilde{e}_{j}\right)\left(\nabla_{\widetilde{e}_{i}}^{T X \otimes F, u} \omega\left(F, h^{F}\right)\right)\left(\widetilde{e}_{j}\right)\right\}_{t, u}^{(4)}(z)\right] . \quad(3.73)
\end{gathered}
$$

Note that by (1.9) and (3.53)-(3.59),

$$
\begin{gathered}
\widetilde{e}_{m}(\sqrt{t u} z)=e_{m} \\
\widetilde{e}_{j}(\sqrt{t u} z)-e_{j}=\mathcal{O}(\sqrt{t u} z) \in T_{y_{0}} Y, \quad \text { for } 1 \leq j \leq m-1 .
\end{gathered}
$$

We deal first with the contribution of $\nabla_{e_{m}}^{2}$ in $L_{t, u}^{(4)}$. Let $\sigma, \sigma^{\prime} \in$ $C_{0, t, u, a}^{\infty}\left(\mathbb{R}_{+}^{m}, K_{r, y_{0}}\right)$ then there exist $\sigma_{1}, \sigma_{2}, \sigma_{1}^{\prime}, \sigma_{2}^{\prime} \in C_{0}^{\infty}\left(\mathbb{R}_{+}^{m}, K_{y_{0}}^{\prime}\right)$, such that $\sigma=\sigma_{1}+e^{m} \wedge \sigma_{2}, \sigma^{\prime}=\sigma_{1}^{\prime}+e^{m} \wedge \sigma_{2}^{\prime}$. Then

$$
\left\langle-\nabla_{e_{m}}^{2} \sigma, \sigma^{\prime}\right\rangle_{t, u, 0}=\sum_{i=1}^{2}\left\langle-\nabla_{e_{m}}^{2} \sigma_{i}, \sigma_{i}^{\prime}\right\rangle_{t, u, 0} .
$$

By (3.44), $\sigma_{2}=\sigma_{2}^{\prime}=0$ on $\mathbb{R}^{m-1} \times\{0\}$, and $\nabla_{e_{m}} \sigma_{1}=\nabla_{e_{m}} \sigma_{1}^{\prime}=0$ on $\mathbb{R}^{m-1} \times\{0\}$, thus for $j=1,2$,

$$
\begin{aligned}
\left\langle-\nabla_{e_{m}}^{2} \sigma_{j}, \sigma_{j}^{\prime}\right\rangle_{t, u, 0} & =\left\langle\nabla_{e_{m}} \sigma_{j}, \nabla_{e_{m}} \sigma_{j}^{\prime}\right\rangle_{t, u, 0} \\
& +\left\langle\nabla_{e_{m}} \sigma_{j}, 2(2 m-r-2)\left(g_{t, u}^{-1} \nabla_{e_{m}} g_{t, u}\right) \sigma_{j}^{\prime}\right\rangle_{t, u, 0} .
\end{aligned}
$$


The families of operators on $H_{t, u, a}^{0}$, given by

$$
1_{\sqrt{t u}|z| \leq 4 \epsilon}\left(w\left(e^{i}\right)-\sqrt{t} i\left(e_{i}\right)\right), \quad 1_{\sqrt{t u}|z| \leq 4 \epsilon}\left(w\left(\widehat{e^{i}}\right)-\sqrt{t} i\left(\widehat{e_{i}}\right)\right),
$$

are uniformly bounded for $t, u \in(0,1]$. We also have $\left|\nabla_{e_{i}} g_{t, u}\right| \leq C$. Using (3.62), (3.67), (3.73), (3.74), and (3.76), we get Theorem 3.12.

If $A \in \mathcal{L}\left(H_{t, u, a}^{k}, H_{t, u, a}^{k^{\prime}}\right), k \geq-1$, we denote by \|\|$_{t, u}^{k, k^{\prime}}$ the norm of $A$ with respect to the norms \|\|$_{t, u, k}$ and \|\|$_{t, u, k^{\prime}}$.

From Theorem 3.12, we get the following analogue of [BiL, Thm. 11.27],

Theorem 3.13. For $u, t \in(0,1]$, there exist constants $\delta_{1}, \delta_{2}>0$ such that the resolvent $\left(\lambda-L_{t, u, a}^{(4)}\right)^{-1}$ exists in

$$
U:=\left\{\lambda \in \mathbb{C} ; \operatorname{Re}(\lambda) \leq \delta_{1} \operatorname{Im}^{2}(\lambda)-\delta_{2}\right\},
$$

and extends to a continuous linear operator from $H_{t, u, a}^{-1}$ into $H_{t, u, a}^{1}$. Moreover, there is $C>0$, independent of $u, t$, and $y_{0} \in Y$, such that

$$
\begin{aligned}
& \left\|\left(\lambda-L_{t, u, a}^{(4)}\right)^{-1}\right\|_{t, u}^{0,0} \leq C, \\
& \left\|\left(\lambda-L_{t, u, a}^{(4)}\right)^{-1}\right\|_{t, u}^{-1,1} \leq C(1+|\lambda|)^{2} .
\end{aligned}
$$

Proof. This is an easy consequence of Theorem 3.12, cf. [BiL, p. 165-167].

Proposition 3.14. For $k \in \mathbb{N}$, there exists $C_{k}>0$ such that for $\sigma, \sigma^{\prime} \in$ $C_{0, t, u, a}^{\infty}\left(\mathbb{R}_{+}^{m}, K_{y_{0}}\right)$

$$
\left|\left\langle\left[\nabla_{e_{i_{1}}},\left[\nabla_{e_{i_{2}}}, \ldots,\left[\nabla_{e_{i_{k}}}, L_{t, u}^{(4)}\right]\right] \ldots\right] \sigma, \sigma^{\prime}\right\rangle_{t, u, 0}\right| \leq C_{k}\|\sigma\|_{t, u, 1}\left\|\sigma^{\prime}\right\|_{t, u, 1},
$$

for any $u, t \in(0,1]$ and $i_{1}, \ldots, i_{k} \in\{1, \ldots, m\}$.

Proof. Observe that $\left[\nabla_{e_{i_{1}}},\left[\nabla_{e_{i_{2}}}, \ldots,\left[\nabla_{e_{i_{k}}}, L_{t, u}^{(4)}\right]\right] \ldots\right]$ has the same structure as $L_{t, u}^{(4)}$, hence we get Proposition 3.14 using the same arguments as in the proof of Theorem 3.12.

We now fix $\delta_{1}, \delta_{2}>0$ as in Theorem 3.13.

Theorem 3.15. For any $k \in \mathbb{N}$, there exist $m_{k} \in \mathbb{N}, C_{k}>0$ such that if $u \in(0,1]$ and $\lambda \in U$, the resolvent $\left(\lambda-L_{t, u, a}^{(4)}\right)^{-1}$ maps $H^{k}\left(\mathbb{R}_{+}^{m}, K_{y_{0}}\right)$ into $H^{k+1}\left(\mathbb{R}_{+}^{m}, K_{y_{0}}\right)$, with norm estimate

$$
\left\|\left(\lambda-L_{t, u, a}^{(4)}\right)^{-1}\right\|_{t, u}^{k, k+1} \leq C_{k}(1+|\lambda|)^{m_{k}} .
$$

Proof. For $1 \leq i \leq m-1, \nabla_{e_{i}}$ preserves the boundary condition (3.44), thus

$$
\left[\nabla_{e_{i}},\left(\lambda-L_{t, u, a}^{(4)}\right)^{-1}\right]=\left(\lambda-L_{t, u, a}^{(4)}\right)^{-1}\left[\nabla_{e_{i}}, L_{t, u, a}^{(4)}\right]\left(\lambda-L_{t, u, a}^{(4)}\right)^{-1} .
$$


The equation (3.81) means that we need to prove that there exist $C_{k}>0, m_{k} \in \mathbb{N}$ such that for $\nabla_{e_{i_{0}}}, \ldots, \nabla_{e_{i_{k}}} \in\left\{\nabla_{e_{i}}\right\}_{i=1}^{m}, s \in C^{\infty}\left(\mathbb{R}_{+}^{m}, K_{y_{0}}\right)$, we have

$$
\left\|\nabla_{e_{i_{0}}} \cdots \nabla_{e_{i_{k}}}\left(\lambda-L_{t, u, a}^{(4)}\right)^{-1} s\right\|_{t, u, 0} \leq C_{k}(1+|\lambda|)^{m_{k}}\|s\|_{t, u, k} .
$$

If $1 \leq i_{0}, \ldots, i_{k} \leq m-1$, then we can express $\nabla_{e_{i_{1}}} \cdots \nabla_{e_{i_{k}}}\left(\lambda-L_{t, u, a}^{(4)}\right)^{-1}$ as a linear combination of operators of the type

$$
\left[\nabla_{e_{j_{1}}}\left[\nabla_{e_{j_{2}}}, \ldots\left[\nabla_{e_{j_{k^{\prime}}}},\left(\lambda-L_{t, u, a}^{(4)}\right)^{-1}\right]\right]\right] \nabla_{e_{j_{k^{\prime}+1}}} \cdots \nabla_{e_{j_{k}}}, \quad k^{\prime}<k .
$$

Let $\mathcal{R}_{t, u}$ be the family operators $\mathcal{R}_{t, u}=\left\{\left[\nabla_{e_{j_{1}}}\left[\nabla_{e_{j_{2}}}, \ldots\left[\nabla_{e_{j_{p}}}, L_{t, u}^{(4)}\right]\right]\right]\right\}$. Clearly, from (3.82), any commutator $\left[\nabla_{e_{j_{1}}}\left[\nabla_{e_{j_{2}}}, \ldots\left[\nabla_{e_{j_{k^{\prime}}}},\left(\lambda-L_{t, u, a}^{(4)}\right)^{-1}\right]\right]\right]$ is a linear combination of operators of the form

$$
\left(\lambda-L_{t, u, a}^{(4)}\right)^{-1} R_{1}\left(\lambda-L_{t, u, a}^{(4)}\right)^{-1} R_{2} \cdots R_{k^{\prime}}\left(\lambda-L_{t, u, a}^{(4)}\right)^{-1}
$$
with $R_{1}, \ldots, R_{k^{\prime}} \in \mathcal{R}_{t, u}$.

By Proposition 3.14, the operators $R_{j} \in \mathcal{R}_{t, u}$ are uniformly bounded in $\mathcal{L}\left(H_{t, u, a}^{1}, H_{t, u, a}^{-1}\right)$. From Theorem 3.13 we see that there exist $C>0$ and $m_{k} \in \mathbb{N}$ such that the norm in $\mathcal{L}\left(H_{t, u, 0}^{0}, H_{t, u, a}^{1}\right)$ of the operators (3.85) is dominated by $C(1+|\lambda|)^{m_{k}}$.

If there are only one $\nabla_{e_{m}}$ in $\nabla_{e_{i_{0}}}, \ldots, \nabla_{e_{i_{k}}}$, then we move this term to the left side, thus we can assume $\nabla_{e_{i_{0}}}=\nabla_{e_{m}}$, and the above proof still gives (3.83).

If there are at least two $\nabla_{e_{m}}$ in $\nabla_{e_{i_{0}}}, \ldots, \nabla_{e_{i_{k}}}$, then we move all $\nabla_{e_{m}}$ to the right side. Now we can express $\nabla_{e_{i_{0}}} \cdots \nabla_{e_{i_{k}}}\left(\lambda-L_{t, u, a}^{(4)}\right)^{-1}$ as

$$
\nabla_{e_{j_{1}}} \cdots \nabla_{e_{j_{l}}}\left(\nabla_{e_{m}}\right)^{k}\left(\lambda-L_{t, u, a}^{(4)}\right)^{-1} \quad k+l \leq m+1 .
$$

For $k<2$, we obtained the estimate (3.83) for this operator. We need to reduce $k \geq 2$ to the case $k<2$.

Let $g_{i j}(z)=\left\langle e_{i}, e_{j}\right\rangle_{g^{T X}}(z)$ and $\left(g^{i j}(z)\right)$ be the inverse of the matrix $\left(g_{i j}(z)\right)_{i, j=1}^{m}$. By $(1.7)$, we know that $g_{m i}(z)=g_{i m}(z)=0, g_{m m}(z)=1$. Thus we have

$$
\Delta^{T X \otimes F, u}=-\left(\nabla_{e_{m}}^{T X \otimes F, u}\right)^{2}-\sum_{i, j=1}^{m-1} g^{i j}(z)\left(\nabla_{e_{i}}^{T X \otimes F, u} \nabla_{e_{j}}^{T X \otimes F, u}-\nabla_{\nabla_{e_{i}}^{T X} e_{j}}^{T X \otimes F, u}\right) .
$$

Thus we know from (3.54) and (3.87), the second order term in $L_{t, u}^{(4)}$ is

$$
-\left(\nabla_{e_{m}}\right)^{2}-\sum_{i, j=1}^{m-1} g^{i j}(\sqrt{t u} z) \nabla_{e_{i}} \nabla_{e_{j}} .
$$

Now, if $k \geq 2$, we replace $\left(\nabla_{e_{m}}\right)^{2}\left(\lambda-L_{t, u, a}^{(4)}\right)^{-1}$ in (3.86) by

$$
1+\left(-\lambda+\left(L_{t, u}^{(4)}+\left(\nabla_{e_{m}}\right)^{2}\right)\right)\left(\lambda-L_{t, u, a}^{(4)}\right)^{-1} .
$$


Now by (3.88), we can continue the processes to get (3.86) with $k \leq 1$. Thus we have proved (3.83).

Now we use Theorem 3.12 and Proposition 3.14 to establish the following uniform estimate (cf. [Bi2, §11h)], [BiL, §11n)]).

Theorem 3.16. For any $k \in \mathbb{N}$ there exist $C_{0}, C>0$ and $r \in \mathbb{N}$ such that

$$
\left|\frac{\partial^{|\alpha|+\left|\alpha^{\prime}\right|}}{\partial z^{\alpha} \partial w^{\alpha^{\prime}}} e^{-L_{t, u, a}^{(4)}}(z, w)\right| \leq C(1+|z|+|w|)^{r} e^{-C_{0}|z-w|^{2}}
$$

uniformly for $t, u \in(0,1]$ and $z, w \in \mathbb{R}_{+}^{m}$, if $|\alpha|,\left|\alpha^{\prime}\right| \leq k$.

Proof. Let $\Gamma$ be the contour in $\mathbb{C}$ defined by

$$
\Gamma=\left\{\lambda \in \mathbb{C} ; \operatorname{Re} \lambda=\delta_{1}(\operatorname{Im} \lambda)^{2}-\delta_{2}\right\} .
$$

Using Theorem 3.13 we find that

$$
\begin{aligned}
e^{-L_{t, u, a}^{(4)}} & =\frac{1}{2 \pi i} \int_{\Gamma} e^{-\lambda}\left(\lambda-L_{t, u, a}^{(4)}\right)^{-1} d \lambda \\
& =\frac{(-1)^{k-1}(k-1) !}{2 \pi i} \int_{\Gamma} e^{-\lambda}\left(\lambda-L_{t, u, a}^{(4)}\right)^{-k} d \lambda .
\end{aligned}
$$

From Theorem 3.15 we deduce that for $k^{\prime} \in \mathbb{N}, \lambda \in U$

$$
\left\|\left(\Delta^{T_{y_{0}} X}\right)^{k^{\prime}}\left(\lambda-L_{t, u, a}^{(4)}\right)^{-2 k^{\prime}}\right\|_{t, u}^{0,0} \leq C_{k^{\prime}} .
$$

Next we study $L_{t, u}^{(4) *}$, the formal adjoint of $L_{t, u}^{(4)}$ with respect to the usual Hermitian product on $L^{2}\left(\mathbb{R}_{+}^{m}, K_{y_{0}}\right): L^{2}\left(\mathbb{R}_{+}^{m}, K_{y_{0}}\right) \ni \sigma, \sigma^{\prime} \mapsto\left\langle\sigma, \sigma^{\prime}\right\rangle=$ $\int_{T_{y_{0}} X}\left\langle\sigma, \sigma^{\prime}\right\rangle d v_{T_{y_{0}} X}(z)$. Then $L_{t, u}^{(4) *}$ has the same structure as the operator $L_{t, u}^{(4)}$ except that the operators $w\left(e^{i}\right), i\left(e_{i}\right), w\left(\widehat{e^{i}}\right), i\left(\widehat{e_{i}}\right)$ are replaced by $i\left(e_{i}\right), w\left(e^{i}\right), i\left(\widehat{e_{i}}\right), w\left(\widehat{e^{i}}\right)$, respectively. For $\sigma \in C^{\infty}\left(\mathbb{R}_{+}^{m}, K_{r, y_{0}}\right)$ we now set

$$
\|\sigma\|_{t, u, 0}^{2}:=\int_{T_{y_{0} X} X}|\sigma|^{2} g_{t, u}(z)^{2(r-2 m+2)} d v_{T_{y_{0}} X}(z) .
$$

We denote by $L_{t, u, a}^{(4) *}$ the operator $L_{t, u}^{(4) *}$ with the dual boundary condition. Then the analysis of the operator $\left(\lambda-L_{t, u, a}^{(4) *}\right)^{-2 k^{\prime}}$ proceeds exactly as before with respect to the new Hilbert norm, in particular (3.93) is true for $L_{t, u, a}^{(4) *}$. We take adjoints again with respect to the ordinary Hermitian product ||$_{L^{2}}$ on $L^{2}\left(\mathbb{R}_{+}^{m}, K_{y_{0}}\right)$. Thus we get for $k^{\prime} \in \mathbb{N}$ and $\lambda \in U$ the estimate

$$
\left\|\left(\Delta_{w}^{T_{y_{0}} X}\right)^{k^{\prime}}\left(\lambda-L_{t, u, a}^{(4)}\right)^{-2 k^{\prime}}(z, w)\right\|_{t, u}^{0,0} \leq C_{k^{\prime}}
$$

Thus from (3.92)-(3.95), for any $k, k^{\prime} \in \mathbb{N}$,

$$
\|\left(\Delta_{z}^{T_{y_{0}} X}\right)^{k}\left(\Delta_{w}^{T_{y_{0}} X}\right)^{k^{\prime}} e^{-L_{t, u, a}^{(4)}(z, w) \|_{t, u}^{0,0} \leq C_{k k^{\prime}} .}
$$


Take $p \in \mathbb{N}$. We equip $L^{2}\left(B_{p+\frac{1}{2}}^{\mathbb{R}_{+}^{m}}(0), K_{y_{0}}\right)$ with the Hermitian product

$$
\sigma, \sigma^{\prime} \in L^{2}\left(B_{p+\frac{1}{2}}^{\mathbb{R}_{+}^{m}}(0), K_{y_{0}}\right) \mapsto\left\langle\sigma, \sigma^{\prime}\right\rangle_{(p)}=\int_{|Z| \leq p+\frac{1}{2}}\left\langle\sigma, \sigma^{\prime}\right\rangle d v_{T_{y_{0}} X}(z),
$$

with norm \|\|$_{(p)}$. If $A \in \mathcal{L}\left(L^{2}\left(B_{p+\frac{1}{2}}^{\mathbb{R}_{+}^{m}}(0), K_{y_{0}}\right)\right)$, let $\|A\|_{(p)}$ be the operator norm of $A$ corresponding to \|\|$_{(p)}$. Clearly, there is $C>0$ such that for any $\sigma$ and $p \in \mathbb{N}$,

$$
\|\sigma\|_{(p)} \leq\|\sigma\|_{t, u, 0}, \quad\|\sigma\|_{t, u, 0} \leq C(1+p)^{4 m-4}\|\sigma\|_{(p)} .
$$

Now, from (3.96), we find that

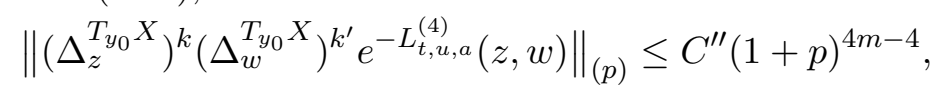

which together with Sobolev's inequality implies that for $k, k^{\prime} \in \mathbb{N}$,

$$
\sup _{|z|,|w| \leq p+\frac{1}{4}} \mid\left(\Delta_{z}^{T_{y_{0}} X}\right)^{k}\left(\Delta_{w}^{T_{y_{0}} X}\right)^{k^{\prime}} e^{-L_{t, u, a}^{(4)}(z, w) \mid \leq C^{\prime \prime}(1+p)^{4 m-4} .}
$$

Using the Sobolev inequality again, we get (3.90) for $C_{0}=0$.

To get the required exponential estimate, we now proceed as in the proof of [Bi2, Thm.11.14]. Let $\mathbb{R} \ni u \mapsto k(u)$ be a smooth even function such that

$$
k(u)=\left\{\begin{array}{lll}
0 & \text { for } & |u| \leq 1 / 2 \\
1 & \text { for } & |u| \geq 1
\end{array}\right.
$$

For $q \in \mathbb{R}_{+}^{*}, a \in \mathbb{C}$, set

$$
K_{q}(a):=2 \int_{0}^{+\infty} \cos (t \sqrt{2} a) \exp \left(-t^{2} / 2\right) k\left(\frac{t}{q}\right) \frac{d t}{\sqrt{2 \pi}} .
$$

Clearly, $K_{q}(a)$ is an even holomorphic function of $a$. Therefore, there is a holomorphic function $a \in \mathbb{C} \rightarrow \widetilde{K}_{q}(a)$ such that

$$
K_{q}(a)=\widetilde{K}_{q}\left(a^{2}\right) \text {. }
$$

Given $c>0$, set

$$
\begin{aligned}
& V_{c}:=\left\{\lambda \in \mathbb{C}, \operatorname{Re}(\lambda) \geq \frac{(\operatorname{Im} \lambda)^{2}}{4 c^{2}}-c^{2}\right\}, \\
& \Gamma_{c}:=\left\{\lambda \in \mathbb{C}, \operatorname{Re}(\lambda)=\frac{(\operatorname{Im} \lambda)^{2}}{4 c^{2}}-c^{2}\right\} .
\end{aligned}
$$

Then by $[\operatorname{Bi2} 2,(11.53)]$, for any $c>0$, there exists $C^{\prime}>0$ such that, given $m, m^{\prime} \in \mathbb{N}$, there exists $C>0$ with

for $q \geq 1$. Also,

$$
\sup _{a \in V_{c}}|a|^{m}\left|\widetilde{K}_{q}^{\left(m^{\prime}\right)}(a)\right| \leq C \exp \left(-C^{\prime} q^{2}\right),
$$

$$
\widetilde{K}_{q}\left(L_{t, u, a}^{(4)}\right)=\frac{1}{2 \pi i} \int_{\Gamma_{c}} \frac{\widetilde{K}_{q}(\lambda)}{\lambda-L_{t, u, a}^{(4)}} d \lambda .
$$


Let $\widetilde{K}_{q}\left(L_{t, u, a}^{(4)}\right)(z, w)$ be the smooth kernel associated to $\widetilde{K}_{q}\left(L_{t, u, a}^{(4)}\right)$ with respect to $d v_{T_{y_{0}} X}(w)$. Using (3.105) and proceeding as before, we get the following analogue of $[\mathrm{Bi} 2,(11.59)]$ : there is $C_{0}>0$ such that for any $k \in \mathbb{N}$, there exist $C>0$ and $r \in \mathbb{N}$ such that

$$
\sup _{|\alpha|,\left|\alpha^{\prime}\right| \leq k}\left|\frac{\partial^{|\alpha|+\left|\alpha^{\prime}\right|}}{\partial z^{\alpha} \partial w^{\alpha^{\prime}}} \widetilde{K}_{q}\left(L_{t, u, a}^{(4)}\right)(z, w)\right| \leq C(1+|z|+|w|)^{r} \exp \left(-C_{0} q^{2}\right),
$$

uniformly for $q \in \mathbb{N}^{*}, z, w \in \mathbb{R}_{+}^{m}$, and $t, u \in(0,1]$.

If $t \geq q$, then $k(t / q)=1$. Using again finite propagation speed for $\cos \left(s \sqrt{L_{t, u, a}^{(4)}}\right)$, we find a constant $C_{0}^{\prime}>0$ such that

$$
e^{-L_{t, u, a}^{(4)}}(z, w)=\widetilde{K}_{q}\left(L_{t, u, a}^{(4)}\right)(z, w) \text { if }|z-w| \geq C_{0}^{\prime} q,
$$

uniformly in $q \in \mathbb{N}^{*}$. From (3.107), (3.108) we deduce that

$$
\begin{aligned}
& \sup _{|\alpha|,\left|\alpha^{\prime}\right| \leq k}\left|\frac{\partial^{|\alpha|+\left|\alpha^{\prime}\right|}}{\partial z^{\alpha} \partial w^{\alpha^{\prime}}} e^{-L_{t, u, a}^{(4)}}(z, w)\right| \\
& \leq C(1+|z|+|w|)^{r} \exp \left(-C_{0} q^{2}\right) \quad \text { if } \quad|z-w| \geq C_{0}^{\prime} q
\end{aligned}
$$

uniformly in $q \in \mathbb{N}^{*}, z, w \in \mathbb{R}_{+}^{m}, t, u \in(0,1]$.

For $z, w \in \mathbb{R}_{+}^{m}$, let $q \in \mathbb{N}$ such that $C_{0}^{\prime} q \leq|z-w| \leq C_{0}^{\prime}(q+1)$. By (3.90) with $C_{0}=0$ and (3.109), we get (3.90).

Let \|\|$_{0, u, 0}$ be the limit norm of \|\|$_{t, u, 0}$ as $t \rightarrow 0$. For $\alpha=\left(\alpha_{1}, \ldots, \alpha_{m}\right) \in \mathbb{Z}_{+}^{m}$ a multindex, we write $z^{\alpha}=z_{1}^{\alpha_{1}} \cdots z_{m}^{\alpha_{m}}$.

Definition 3.17. For $k \in\{-1,0,1\}$ and $k^{\prime} \in \mathbb{N}$, let $H_{0, u}^{k, k^{\prime}}$ be the set of $\sigma \in H^{k}\left(\mathbb{R}_{+}^{m}, K_{y_{0}}\right)$ such that $z^{\alpha} \sigma \in H^{k}\left(\mathbb{R}_{+}^{m}, K_{y_{0}}\right)$ for $|\alpha| \leq k^{\prime}$. For $\sigma \in H_{0, u}^{k, k^{\prime}}$ we write

$$
\|\sigma\|_{0, u,\left(k, k^{\prime}\right)}^{2}:=\sum_{|\alpha| \leq k^{\prime}}\left\|z^{\alpha} \sigma\right\|_{0, u, k}^{2} .
$$

Theorem 3.18. There exist $C>0$ and $k, k^{\prime} \in \mathbb{N}$ such that $\left\|\left(\left(\lambda-L_{t, u, a}^{(4)}\right)^{-1}-\left(\lambda-L_{0, u, a}^{(4)}\right)^{-1}\right) \sigma\right\|_{t, u, 0} \leq C t^{1 / 2}(1+|\lambda|)^{k}\|\sigma\|_{0, u,\left(0, k^{\prime}\right)}$, uniformly in $t, u \in(0,1], \lambda \in U$, and $\sigma \in L^{2}\left(\mathbb{R}_{+}^{m}, K_{y_{0}}\right)$.

Proof. Note that from (3.44), the boundary condition is fixed for $t, u \in$ $(0,1]$, thus if $\sigma^{\prime} \in C^{\infty}\left(\mathbb{R}_{+}^{m}, K_{y_{0}}\right)$ is in $\operatorname{Dom}\left(L_{0, u, a}^{(4)}\right)$, then $\sigma^{\prime} \in \operatorname{Dom}\left(L_{t, u, a}^{(4)}\right)$. Thus

$\left(\lambda-L_{t, u, a}^{(4)}\right)^{-1}-\left(\lambda-L_{0, u, a}^{(4)}\right)^{-1}=\left(\lambda-L_{t, u, a}^{(4)}\right)^{-1}\left(L_{t, u}^{(4)}-L_{0, u}^{(4)}\right)\left(\lambda-L_{0, u, a}^{(4)}\right)^{-1}$.

Observe that \|\|$_{t, u, 0} \leq\|\|_{0, u, 0}$. For $\sigma \in C_{0,0, u, a}^{\infty}\left(\mathbb{R}_{+}^{m}, K_{y_{0}}\right), \sigma^{\prime} \in$ $C_{0, t, u, a}^{\infty}\left(\mathbb{R}_{+}^{m}, K_{y_{0}}\right)$, we know by (3.73) and the arguments in the proof of Theorem 3.12,

$$
\left|\left\langle\left(L_{t, u}^{(4)}-L_{0, u}^{(4)}\right) \sigma, \sigma^{\prime}\right\rangle_{t, u, 0}\right| \leq C t^{1 / 2}\|\sigma\|_{0, u,(1,4)}\left\|\sigma^{\prime}\right\|_{t, u, 1},
$$


hence

$$
\left\|\left(L_{t, u}^{(4)}-L_{0, u}^{(4)}\right) \sigma\right\|_{t, u,-1} \leq C t^{1 / 2}\|\sigma\|_{0, u,(1,4)} .
$$

Now after taking the limit, Theorem 3.13 still holds for $t=0$. Set $\widetilde{z}_{j}=z_{j}$ for $1 \leq j \leq m-1$, and $\widetilde{z}_{m}=\left(1-\varphi\left(z_{m}\right)\right) z_{m}$. From (3.73), for $i_{1}, \ldots, i_{p} \in\{1, \ldots, m\}$, we get

$$
\left\|\left[\widetilde{z}_{i_{1}},\left[\widetilde{z}_{i_{2}} \cdots\left[\widetilde{z}_{i_{p}}, L_{t, u}^{(4)}\right]\right]\right]\right\|_{0, u}^{1,0} \leq C_{p} .
$$

Now by (3.114) and $\widetilde{z}_{j}(1 \leq i \leq m)$ preserves the boundary condition (3.44), as in (3.81), we know that for $k \in \mathbb{N}$, there exist $C>0, k^{\prime} \in \mathbb{N}$ such that for $u \in(0,1], \lambda \in U$, if $\sigma \in C_{0,0, u, a}^{\infty}\left(\mathbb{R}_{+}^{m}, K_{y_{0}}\right)$,

$$
\left\|\left(\lambda-L_{0, u, a}^{(4)}\right)^{-1} \sigma\right\|_{0, u,(1, k)} \leq C(1+|\lambda|)^{k^{\prime}}\|\sigma\|_{0, u,(0, k)} .
$$

Now (3.111) follows from Theorem 3.13, (3.112), (3.113), and (3.115).

Observe that the norm ||$_{L^{2}}$ is smaller than \|\|$_{t, u, 0}$, thus $e^{-L_{t, u, a}^{(4)}} \rightarrow$ $e^{-L_{0, u, a}^{(4)}}$ in the strong topology. Using the uniform bounds in Theorem 3.16, we get

Theorem 3.19. For $z, w \in \mathbb{R}_{+}^{m}$,

$$
\lim _{t \rightarrow 0} e^{-L_{t, u, a}^{(4)}}(z, w)=e^{-L_{0, u, a}^{(4)}}(z, w) .
$$

Theorem 3.20. There exist $r \in \mathbb{N}, C_{0}>0$ such that for any $l \in \mathbb{N}$, there exists $C>0$ such that for $t \in(0,1]$ and $z, w \in \mathbb{R}_{+}^{m}$,

$$
\left|z_{m}^{l}\left(e^{-L_{t, a}^{(3)}}-e^{-L_{t}^{(3)}}\right)(z, w)\right| \leq C(1+|z|+|w|)^{r} \exp \left(-C_{0}|z-w|^{2}\right) .
$$

Proof. Let $J^{*}$ be the restriction of the functions on $\mathbb{R}^{m}$ to $\mathbb{R}_{+}^{m}$. Observe first that $\left(\lambda-L_{t, a}^{(3)}\right)^{-1}\left(\lambda-L_{t, a}^{(3)}\right) \sigma=\sigma$ holds only if $\sigma \in \operatorname{Dom}\left(L_{t, a}^{(3)}\right)$. Thus with $\varphi$ from (3.39),

$$
\begin{aligned}
& \left(\lambda-L_{t, a}^{(3)}\right)^{-1} J^{*}-J^{*}\left(\lambda-L_{t}^{(3)}\right)^{-1}=\left(\lambda-L_{t, a}^{(3)}\right)^{-1} J^{*}\left(\lambda-L_{t}^{(3)}\right)\left(\lambda-L_{t}^{(3)}\right)^{-1} \\
& +\varphi\left(2 z_{m}\right) J^{*}\left(\lambda-L_{t}^{(3)}\right)^{-1}-\left(\lambda-L_{t, a}^{(3)}\right)^{-1}\left(\lambda-L_{t}^{(3)}\right)\left(1-\varphi\left(2 z_{m}\right)\right) J^{*}\left(\lambda-L_{t}^{(3)}\right)^{-1} \\
& \quad=\varphi\left(2 z_{m}\right) J^{*}\left(\lambda-L_{t}^{(3)}\right)^{-1}+\left(\lambda-L_{t, a}^{(3)}\right)^{-1} \varphi\left(2 z_{m}\right) J^{*} \\
& \quad+\left(\lambda-L_{t, a}^{(3)}\right)^{-1}\left[\varphi\left(2 z_{m}\right), L_{t}^{(3)}\right] J^{*}\left(\lambda-L_{t}^{(3)}\right)^{-1}, \\
& \left(\lambda-L_{t, a}^{(3)}\right)^{-k}-J^{*}\left(\lambda-L_{t}^{(3)}\right)^{-k} J^{*} \\
& \quad=\sum_{j=0}^{k-1}\left(\lambda-L_{t, a}^{(3)}\right)^{-j}\left(\left(\lambda-L_{t, a}^{(3)}\right)^{-1} J^{*}-J^{*}\left(\lambda-L_{t}^{(3)}\right)^{-1}\right)\left(\lambda-L_{t}^{(3)}\right)^{-k+j+1} J^{*}
\end{aligned}
$$

Now for $k \geq 1$, 


$$
\begin{aligned}
& \widetilde{z}_{m}^{l}\left(e^{-L_{t, a}^{(3)}}-e^{-L_{t}^{(3)}}\right)(z, w) \\
& =\frac{(-1)^{k-1}(k-1) !}{2 \pi i} \int_{\Gamma} e^{-\lambda} \widetilde{z}_{m}^{l}\left(\left(\lambda-L_{t, a}^{(3)}\right)^{-k}-J^{*}\left(\lambda-L_{t}^{(3)}\right)^{-k} J^{*}\right) d \lambda .
\end{aligned}
$$

By using $\widetilde{z}_{m} \varphi\left(2 z_{m}\right)=0,(3.114),(3.118),(3.119)$ and proceeding as in the proof of (3.100), we get : there exists $r>0$ such that for any $l \in \mathbb{N}$, there exists $C>0$ such that

$$
\left|\widetilde{z}_{m}^{l}\left(e^{-L_{t, a}^{(3)}}-e^{-L_{t}^{(3)}}\right)(z, w)\right| \leq C(1+|z|+|w|)^{r} .
$$

From Theorems 3.9, 3.16 and (3.120), we get (3.117).

3.7 The model problem on $\mathbb{R}_{+}=[0, \infty)$. In this subsection, we find the explicit fundamental solution of the model problem (3.134) for $i=4$. The following formula is due to Sommerfeld (cf. [CaJ, §14.2]) and was also used in [APS, §4].

Proposition 3.21. Consider the boundary value problem for $-\partial^{2} / \partial x^{2}$ in $L^{2}\left(\mathbb{R}_{+}, \mathbb{C}^{n}\right)$ with domain

$$
\left\{\sigma \in C_{0}^{\infty}\left(\mathbb{R}_{+}, \mathbb{C}^{n}\right) ;\left.\left(\frac{\partial}{\partial x}+\alpha\right) \sigma\right|_{x=0}=0\right\},
$$

where $\alpha$ is a $n \times n$ matrix. Then the heat kernel, $F_{\alpha}(t, x, \xi)$, of this problem is given by

$F_{\alpha}(t, x, \xi)=\frac{1}{\sqrt{4 \pi t}}\left(e^{-\frac{(x-\xi)^{2}}{4 t}}+e^{-\frac{(x+\xi)^{2}}{4 t}}\right)+\frac{\alpha}{\sqrt{\pi t}} \int_{0}^{+\infty} e^{-\frac{(x+y+\xi)^{2}}{4 t}} e^{\alpha y} d y$.

Note that the heat kernel, $F_{D i r}$, for the Dirichlet boundary condition is given by

$$
F_{\text {Dir }}(t, x, \xi)=\frac{1}{\sqrt{4 \pi t}}\left(e^{-\frac{(x-\xi)^{2}}{4 t}}-e^{-\frac{(x+\xi)^{2}}{4 t}}\right) .
$$

For the integral in (3.121), we have if $\xi=x$

$$
\begin{aligned}
& \frac{\alpha}{\sqrt{\pi t}} \int_{0}^{\infty} \int_{0}^{\infty} e^{-\frac{(y+2 x)^{2}}{4 t}} e^{\alpha y} d y d x=2 \sqrt{\frac{t}{\pi}} \alpha \int_{0}^{\infty} \int_{0}^{\infty} e^{-(x+y)^{2}} e^{2 \sqrt{t} \alpha y} d y d x \\
& =2 \sqrt{\frac{t}{\pi}} \alpha \int_{0}^{\infty} d z e^{-z^{2}} \int_{0}^{z} e^{2 \sqrt{t} \alpha y} d y \\
& \quad=\frac{1}{\sqrt{\pi}} \int_{0}^{\infty} e^{-z^{2}}\left(e^{2 \sqrt{t} \alpha z}-1\right) d z=\sum_{k=1}^{\infty} \frac{\alpha^{k} t^{\frac{k}{2}}}{2 \Gamma\left(\frac{k}{2}+1\right)}
\end{aligned}
$$

Likewise, in the sense of power series

$$
\begin{aligned}
& \frac{\alpha}{\sqrt{\pi t}} \int_{0}^{\infty} \int_{0}^{\infty} e^{-\frac{(y+2 x)^{2}}{4 t}} e^{\alpha y} x d y d x=\frac{2 t \alpha}{\sqrt{\pi}} \int_{0}^{\infty} d z e^{-z^{2}} \int_{0}^{z} e^{2 \sqrt{t} \alpha y}(z-y) d y \\
& =\sqrt{\frac{t}{\pi}} \int_{0}^{\infty} e^{-z^{2}}\left[\frac{1}{2 \sqrt{t} \alpha}\left(e^{2 \sqrt{t} \alpha z}-1\right)-z\right] d z=\sum_{k=1}^{\infty} \frac{\alpha^{k-1} t^{\frac{k}{2}}}{4 \Gamma\left(\frac{k}{2}+1\right)}-\frac{\sqrt{t}}{2 \sqrt{\pi}} .
\end{aligned}
$$


Thus by (3.121), (3.122), and (3.123):

$$
\int_{0}^{\infty}\left(F_{\alpha}-F_{D i r}\right)(t, x, x) d x=\frac{1}{\sqrt{\pi}} \int_{0}^{\infty} e^{-z^{2}} e^{2 \sqrt{t} \alpha z} d z=\sum_{k=0}^{\infty} \frac{\alpha^{k} t^{\frac{k}{2}}}{2 \Gamma\left(\frac{k}{2}+1\right)} .
$$

For later purposes, we also note the following identity resulting from (3.121), (3.122), and (3.124):

$$
\begin{aligned}
\int_{0}^{\infty} x\left(F_{\alpha}-F_{D i r}\right)(t, x, x) d x & =\frac{1}{\sqrt{\pi}} \int_{0}^{\infty} e^{-z^{2}} \frac{1}{2 \alpha}\left(e^{2 \sqrt{t} \alpha z}-1\right) d z \\
& =\sum_{k=1}^{\infty} \frac{\alpha^{k-1} t^{\frac{k}{2}}}{4 \Gamma\left(\frac{k}{2}+1\right)} .
\end{aligned}
$$

3.8 Proof of Theorem 3.2. Let $e^{-u L_{0, a}^{(3)}}(z, w), e^{-u L_{0}^{(3)}}(z, w)$ be the smooth kernels of $e^{-u L_{0, a}^{(3)}}, e^{-u L_{0}^{(3)}}$ with respect to $d v_{T_{y_{0}} X}(w)$. For $z^{\prime}, w^{\prime} \in$ $\mathbb{R}^{m-1}$, set

$$
K\left(u, z^{\prime}, w^{\prime}\right)=(4 \pi u)^{-\frac{m-1}{2}} \exp \left(-\frac{\left|z^{\prime}-w^{\prime}\right|^{2}}{4 u}\right) .
$$

By Theorems 3.10, 3.19, 3.20, (3.11), (3.48), and (3.51), we get $\lim _{t \rightarrow 0} I_{t}\left(y_{0}\right)=\int_{0}^{\infty} 2^{m-1}(-1)^{\frac{m(m-1)}{2}} \operatorname{Tr}_{s}\left[\left[P_{0}^{(3)}\left(\left(0, z_{m}\right),\left(0, z_{m}\right)\right)\right]^{\max }\right] d v_{N}\left(z_{m}\right)$.

Let $\left.Q(t, z, w) \in\left(\Lambda\left(T^{*} Y\right) \widehat{\otimes} \widehat{\Lambda\left(T^{*} Y\right.}\right) \widehat{\otimes} \operatorname{End}\left(\Lambda\left(N^{*}\right) \otimes F\right)\right)_{y_{0}}\left(z, w \in \mathbb{R}_{+}^{m}\right.$, $t>0)$ be the fundamental solution of the following problem with respect to $d v_{T_{y_{0}} X}(w)$,

$$
\left\{\begin{array}{l}
\left.i\left(e_{m}\right) \omega\right|_{z_{m}=0}=0 \\
\left.w\left(e^{m}\right)\left[\nabla_{e_{m}}+\dot{S}\left(y_{0}\right)\right] \omega\right|_{z_{m}=0}=0 \\
\left(\frac{\partial}{\partial t}+\Delta^{T_{y_{0}} X}\right) \omega=0 .
\end{array}\right.
$$

Then we get from (3.52) and (3.129)

$$
\begin{aligned}
& e^{-u L_{0, a}^{(3)}}(z, w)=e^{z_{m} \dot{S}\left(y_{0}\right)} Q(u, z, w) \exp \left(-\frac{u}{2}\left(\dot{R}^{T X} \mid Y\right)_{y_{0}}\right) e^{-w_{m} \dot{S}\left(y_{0}\right)}, \\
& e^{-u L_{0}^{(3)}}(z, w) \\
& \quad=(4 \pi u)^{-m / 2} \exp \left(-\frac{|z-w|^{2}}{4 u}\right) \exp \left(-\frac{u}{2}\left(\dot{R}^{T X} \mid Y\right)_{y_{0}}\right) e^{\left(z_{m}-w_{m}\right) \dot{S}\left(y_{0}\right)} .
\end{aligned}
$$

Note that on $\Lambda\left(N^{*}\right)$,

$$
\operatorname{Tr}_{s}\left[w\left(e^{m}\right) i\left(e_{m}\right)\right]=-\operatorname{Tr}_{s}\left[i\left(e_{m}\right) w\left(e^{m}\right)\right]=-1 .
$$

Decompose

$$
Q=: Q_{1} w\left(e^{m}\right)+Q_{2} i\left(e_{m}\right)+Q_{3} w\left(e^{m}\right) i\left(e_{m}\right)+Q_{4} i\left(e_{m}\right) w\left(e^{m}\right) .
$$

with $\left.Q_{i} \in\left(\Lambda\left(T^{*} Y\right) \widehat{\otimes} \widehat{\Lambda\left(T^{*} Y\right.}\right) \otimes \operatorname{End}(F)\right)_{y_{0}}$. By (3.47), (3.130), (3.131), and (3.132), 


$$
\begin{aligned}
& \operatorname{Tr}_{s}\left[\left[P_{0}^{(3)}\left(\left(0, z_{m}\right),\left(0, z_{m}\right)\right)\right]^{\max }\right] \\
= & \operatorname{Tr}_{F}\left[\left[\left(Q_{4}-Q_{3}\right)\left(1,\left(0, z_{m}\right),\left(0, z_{m}\right)\right) \exp \left(-\frac{1}{2}\left(\dot{R}^{T X} \mid Y\right)_{y_{0}}\right)\right]^{\max }\right] .
\end{aligned}
$$

Thus it remains to determine $Q_{i}$. Let $\psi$ be a smooth section of $\left(\Lambda\left(T^{*} Y\right) \widehat{\otimes}\left(\widehat{\Lambda\left(T^{*} Y\right.}\right) \otimes F\right)_{y_{0}}$ with compact support in $\mathbb{R}_{+}$. Then for $i=1,2$, $\left.Q_{i} \psi\right|_{t=0}=0$, by the uniqueness of the solution and (3.129), we know that $Q_{1}=Q_{2}=0$. Now, from (3.129), we have for $i=3,4$

$$
\left\{\begin{array}{l}
\left.Q_{i} \psi\right|_{t=0}=\psi, \\
\left.Q_{3} \psi\right|_{z_{m}=0}=0,\left.\quad\left(\nabla_{e_{m}}+\dot{S}\left(y_{0}\right)\right) Q_{4} \psi\right|_{z_{m}=0}=0, \\
\left(\frac{\partial}{\partial t}+\Delta^{T_{y_{0}} X}\right) Q_{i} \psi=0 .
\end{array}\right.
$$

By separation of variables and Proposition 3.21, we get

$$
\begin{aligned}
& Q_{3}(t, z, w)=K\left(t, z^{\prime}, w^{\prime}\right) F_{D i r}\left(t, z_{m}, w_{m}\right), \\
& Q_{4}(t, z, w)=K\left(t, z^{\prime}, w^{\prime}\right) F_{\dot{S}}\left(t, z_{m}, w_{m}\right) .
\end{aligned}
$$

Thus using (1.1), (1.17), (3.128), (3.133), (3.135), and (3.125) we arrive at

$$
\begin{aligned}
\lim _{t \rightarrow 0} I_{t}(y) d v_{Y}(y) & =\operatorname{rk}(F) \int^{B_{Y}} \exp \left(-\frac{1}{2}\left(\dot{R}^{T X} \mid Y\right)_{y}\right) \sum_{k=0}^{\infty} \frac{\dot{S}(y)^{k}}{2 \Gamma\left(\frac{k}{2}+1\right)} \\
& =(-1)^{m-1} \operatorname{rk}(F) e_{b}\left(Y, \nabla^{T X}\right) .
\end{aligned}
$$

Now (3.11), (3.14), and (3.136) add up to the proof of Theorem 3.2.

\section{Ray-Singer Metrics and Anomaly Formula}

The purpose of this section and the next is to establish the anomaly formula for Ray-Singer metrics. By the estimate (3.12), we can split the problem into an interior part and a contribution from the boundary. In the interior of $X$, we can apply directly [BiZ1, §4], so our main contribution consists in determining the boundary contribution. In this section, we prove Theorem 0.1 only under the assumption (4.30), while in section 5 , we will prove Theorem 0.1 in full generality.

This section is organized as follows. In subsection 4.1, we define the Ray-Singer metric of manifolds with boundary. In subsection 4.2, we state a formula evaluating the variation of Ray-Singer metrics as the constant term in the asymptotic expansion of the supertrace of a heat kernel multiplied by an endomorphism. In subsection 4.3, we reduce the problem to a problem near the boundary $Y$ and in subsection 4.4, we prove Theorem 0.1 under the assumption (4.30). In subsection 4.5, we compare our results and LückSchick's examples [LüS, Appen. A].

We use the notation established in sections $1.3,3$. Recall that $X$ is a compact manifold with smooth boundary $\partial X=Y$ and $\operatorname{dim} X=m$. Let $F$ 
be a flat complex vector bundle on $X$ with flat connection $\nabla^{F}$. Let $h^{F}$ be a Hermitian metric on $F$ and let $g^{T X}$ be a Riemannian metric on $T X$.

4.1 Ray-Singer metrics on $\operatorname{det} \boldsymbol{H}^{\bullet}(\boldsymbol{X}, \boldsymbol{F})$. Let $P_{a}$ be the orthogonal projection in $L^{2}\left(X, \Lambda\left(T^{*} X\right) \otimes F\right)$ onto $H_{a}^{\bullet}(X, F)$ with $P_{a}^{\perp}:=1-P_{a}$, and let $N$ be the number operator on $\Lambda\left(T^{*} X\right) \otimes F$, which is multiplication by $p$ on $\Lambda^{p}\left(T^{*} X\right) \otimes F$.

Definition 4.1. For $u \in \mathbb{C}$ with $\operatorname{Re}(u)>\frac{1}{2} \operatorname{dim} X$, set

$$
\begin{aligned}
\theta_{a}^{F}(u): & =-\operatorname{Tr}_{s}\left[N\left(D_{a}^{2}\right)^{-u} P_{a}^{\perp}\right] \\
& =-\frac{1}{\Gamma(u)} \int_{0}^{\infty} t^{u} \operatorname{Tr}_{s}\left[N \exp \left(-t D_{a}^{2}\right) P_{a}^{\perp}\right] \frac{d t}{t} .
\end{aligned}
$$

Theorem 4.2. When $t \rightarrow 0$, for any $k \in \mathbb{N}$,

and

$$
\operatorname{Tr}_{s}\left[N \exp \left(-t^{2} D_{a}^{2}\right)\right]=\sum_{j=-1}^{k} a_{j} t^{j}+\mathcal{O}\left(t^{k+1}\right),
$$

$$
\begin{aligned}
& a_{-1}=\operatorname{rk}(F) \int_{X} \int_{i=1}^{B_{X}} \sum^{m} \frac{1}{2} e^{i} \wedge \widehat{e^{i}} \wedge \exp \left(-\frac{1}{2} \dot{R}^{T X}\right) \\
& +\operatorname{rk}(F) \int_{Y} \int^{B_{Y}} \sum_{\alpha=1}^{m-1} \frac{1}{2} e^{\alpha} \wedge \widehat{e^{\alpha}} \wedge \sum_{k=0}^{\infty} \frac{\dot{S}^{k}}{2 \Gamma\left(\frac{k}{2}+1\right)} \exp \left(-\frac{1}{2}\left(\dot{R}^{T X} \mid Y\right)\right), \\
& a_{0}=\frac{m}{2} \chi(X, F) .
\end{aligned}
$$

Theorem 4.2 generalizes [BiZ1, Thm. 7.10] to manifolds with boundary; it will be proved in subsection 5.6 and it is used only for Definition 4.3 in this paper. Thus $\theta_{a}^{F}(u)$ extends to a meromorphic function in $\mathbb{C}$ which is holomorphic at 0 in view of Theorem 4.2.

Let $\operatorname{det} H^{\bullet}(X, F)$ be the determinant line of the cohomology $H^{\bullet}(X, F)$ which is the complex line given by $\operatorname{det} H^{\bullet}(X, F)=\bigotimes_{p=0}^{m}\left(\operatorname{det} H^{p}(X, F)\right)^{(-1)^{p}}$. By the isomorphism in (3.6), $H^{\bullet}(X, F)$ is naturally equipped with a $L^{2}$-metric $h^{H(X, F)}$ induced from the scalar product $\langle\cdot, \cdot\rangle$ on $\Omega(X, F)$; | $\left.\right|_{\operatorname{det} H \bullet(X, F)} ^{L^{2}}$ denote the induced metric on $\operatorname{det} H^{\bullet}(X, F)$.

DeFinition 4.3. The analytic torsion of $X$ with coefficients in $F$ is defined by $T_{a}\left(X, h^{F}\right):=\exp \left\{\frac{1}{2} \frac{\partial \theta_{a}^{F}}{\partial u}(0)\right\}$, and the Ray-Singer metric on the line $\operatorname{det} H^{\bullet}(X, F)$ is defined by

$$
\|\|_{\operatorname{det} H \bullet(X, F)}^{R S}:=T_{a}\left(X, h^{F}\right)||_{\operatorname{det} H \bullet(X, F)}^{L^{2}} \cdot
$$

Definition 4.4 (cf. Definition 3.4, [BiZ1, Def. 4.5]). We introduce a real closed 1-form on $X$ by

$$
\theta\left(F, h^{F}\right):=\operatorname{Tr}\left[\left(h^{F}\right)^{-1}\left(\nabla^{F} h^{F}\right)\right] .
$$


4.2 An infinitesimal variation formula for the Ray-Singer metric. Let $\mathbb{R} \ni s \mapsto\left(g_{s}^{T X}, h_{s}^{F}\right)$ be a smooth family of metrics on $T X$ and $F$ and let $*_{s}, *_{s}^{F}$ be the Hodge operators associated to the metrics $g_{s}^{T X},\left(g_{s}^{T X}, h_{s}^{F}\right)$. Let $D_{s}$ be the operator $D$ defined in (3.4) attached to the metrics $\left(g_{s}^{T X}, h_{s}^{F}\right)$. Let \|\|$_{\operatorname{det} H \bullet(X, F), s}^{R S}$ be the corresponding Ray-Singer metric on $\operatorname{det} H^{\bullet}(X, F)$.

The following theorem is an extension of [BiZ1, Thm.4.14], where the case $\partial X=Y=\emptyset$ was treated.

Theorem 4.5. As $t \rightarrow 0$, for any $k \in \mathbb{N}$, there is an asymptotic expansion

$$
\operatorname{Tr}_{s}\left[\left(*_{s}^{-1} \frac{\partial *_{s}}{\partial s}+\left(h_{s}^{F}\right)^{-1} \frac{\partial h_{s}^{F}}{\partial s}\right) e^{-t D_{s, a}^{2}}\right]=\sum_{j=-m}^{k} M_{j, s} t^{j / 2}+\mathcal{O}\left(t^{(k+1) / 2}\right),
$$

with

$$
M_{0, s}=\frac{\partial}{\partial s} \log \left(\left(\|\|_{\operatorname{det} H \bullet(X, F), s}^{R S}\right)^{2}\right) .
$$

Proof. The equation (4.6) is an immediate consequence of the results in [S], [Gr] on the asymptotics of the heat kernel on manifolds with boundary.

When $h_{s}^{F}=h^{F}$ is a fixed flat metric on $F$, the equation (4.7) is [Ch, Thm. 3.27], [RS, Thm.7.3] which was proved by using [Ch, Thm. 3.10], [RS, Prop. 6.1]. Especially, [Ch, Thm. 3.27] works with no special assumptions on the behavior of the normals at the boundary (i.e. it is not assumed that the unit normal of $Y$ with respect to $g_{s}^{T X}$ is independent of the parameter $s$ ). To be complete, we give a proof in the general case by combining Cheeger's trick and the proof of [BiGS2, Thm. 1.18].

In the sequel, let $\left\{e_{s, j}\right\}$ be an orthonormal basis of $\left(T X, g_{s}^{T X}\right)$. Let $d_{s}^{F *}$ be the formal adjoint of $d^{F}$ for the metrics $g_{s}^{T X}$ and $h_{s}^{F}$. Since $\nabla^{T X}$ is torsion free, we obtain from (3.25) (cf. [BiLo, (3.42)]),

$$
d_{s}^{F *}=\sum_{j=1}^{m}\left(-i\left(e_{s, j}\right) \nabla_{e_{s, j}}^{T X \otimes F}-i\left(e_{s, j}\right) \omega\left(F, h_{s}^{F}\right)\left(e_{s, j}\right)\right) .
$$

By (1.10), (3.23), (4.8), as in (3.36),

$$
\begin{aligned}
i\left(e_{s, m}\right) d_{s}^{F *}= & -i\left(e_{s, m}\right) \sum_{\alpha=1}^{m-1} i\left(e_{s, \alpha}\right)\left({ }^{s p} \nabla_{e_{s, \alpha}}^{T X \otimes F}+\omega\left(F, h_{s}^{F}\right)\left(e_{s, \alpha}\right)\right. \\
& \left.+\left\langle e_{s, m}, A_{s}\left(e_{s, \alpha}\right) e_{s, \beta}\right\rangle w\left(e_{s}^{m}\right) i\left(e_{s, \beta}\right)\right) \\
= & -i\left(e_{s, m}\right) \sum_{\alpha=1}^{m-1} i\left(e_{s, \alpha}\right)\left({ }^{s p} \nabla_{e_{s, \alpha}}^{T X \otimes F}+\omega\left(F, h_{s}^{F}\right)\left(e_{s, \alpha}\right)\right) .
\end{aligned}
$$

Then from (4.9), we get

$$
\left.i\left(e_{s, m}\right) d_{s}^{F *} \sigma\right|_{Y}=0 \quad \text { if }\left.i\left(e_{s, m}\right) \sigma\right|_{Y}=0 .
$$


Set

$$
Q_{s}=-\left(*_{s}^{F}\right)^{-1} \frac{\partial *_{s}^{F}}{\partial s}=-\left(*_{s}^{-1} \frac{\partial *_{s}}{\partial s}+\left(h_{s}^{F}\right)^{-1} \frac{\partial h_{s}^{F}}{\partial s}\right) .
$$

Then by (3.3),

$$
\frac{\partial}{\partial s} d_{s}^{F *}=-\left[d_{s}^{F *}, Q_{s}\right] .
$$

Now by using (4.10) and Cheeger's trick [Ch, p. 277-278] to avoid a technical problem with the boundary condition, we get (cf. also [BiGS2, (1.113)] for manifolds without boundary)

$$
\frac{\partial}{\partial s} \operatorname{Tr}_{s}\left[N \exp \left(-t D_{s, a}^{2}\right)\right]=-t \frac{\partial}{\partial t} \operatorname{Tr}_{s}\left[Q_{s} \exp \left(-t D_{s, a}^{2}\right)\right] .
$$

Set $K_{s}=\oplus_{p=0}^{m} K_{s}^{p}=\operatorname{Ker} D_{s, a}$. Let $P_{s, a}$ be the orthogonal projection operator from $\Omega(X, F)$ on $\operatorname{Ker} D_{s, a}$ for the Hermitian product \langle\rangle$_{s}$ on $\Omega(X, F)$ associated with $g_{s}^{T X}$ and $h_{s}^{F}$ defined in (3.2). As $\operatorname{dim} K_{s}^{p}$ doesn't depend on $s$, from $(4.1),(4.13)$, and $\operatorname{Tr}_{s}\left[Q_{s}\left(\exp \left(-t D_{s, a}^{2}\right)-P_{s, a}\right)\right]$ decays exponentially when $t \rightarrow \infty$, we obtain for $\operatorname{Re}(u)$ large enough,

$$
\begin{aligned}
\frac{\partial}{\partial s} \theta_{s, a}(u) & =\frac{1}{\Gamma(u)} \int_{0}^{\infty} t^{u} \frac{\partial}{\partial t} \operatorname{Tr}_{s}\left[Q_{s} \exp \left(-t D_{s, a}^{2}\right)\right] d t \\
& =\frac{-u}{\Gamma(u)} \int_{0}^{\infty} t^{u-1} \operatorname{Tr}_{s}\left[Q_{s}\left(\exp \left(-t D_{s, a}^{2}\right)-P_{s, a}\right)\right] d t
\end{aligned}
$$

Using (4.14), we find

$$
\frac{\partial}{\partial s}\left(\frac{\partial}{\partial u} \theta_{s, a}\right)(0)=M_{0, s}+\operatorname{Tr}_{s}\left[Q_{s} P_{s, a}\right] .
$$

The line bundle, $\operatorname{det} H^{\bullet}(X, F)$, is canonically identified with $\lambda_{s}=$ $\otimes_{p=0}^{m}\left(\operatorname{det} K_{s}^{p}\right)^{(-1)^{p}}$. Under this identification, the canonical isomorphism of the line bundle $\phi_{s}: \lambda_{0} \rightarrow \lambda_{s}$ is defined by $\phi_{s}(\sigma)=P_{s, a}(\sigma)$ for $\sigma \in \lambda_{0}$.

If $\sigma$ and $\sigma^{\prime}$ are forms in the kernel of $D_{s, a}^{2}$, we have by definition

$$
\left\langle P_{s, a} \sigma, P_{s, a} \sigma^{\prime}\right\rangle_{s}=\int_{X} P_{s, a} \sigma \wedge *_{s}^{F} P_{s, a} \sigma^{\prime}
$$

From $P_{s, a}^{2}=P_{s, a}$, we get $\left(\frac{\partial}{\partial s} P_{s, a}\right) P_{s, a}+P_{s, a}\left(\frac{\partial}{\partial s} P_{s, a}\right)=\frac{\partial}{\partial s} P_{s, a}$, thus the operator $\frac{\partial}{\partial s} P_{s, a}$ sends $\operatorname{Ker} D_{s, a}$ to its orthogonal complement for $\langle\cdot, \cdot\rangle_{s}$. Therefore, from (4.16), we get

$$
\frac{\partial}{\partial s}\left\langle P_{s, a} \sigma, P_{s, a} \sigma^{\prime}\right\rangle_{s}=\int_{X} P_{s, a} \sigma \wedge\left(\frac{\partial}{\partial s} *_{s}^{F}\right) P_{s, a} \sigma^{\prime}=-\left\langle P_{s, a} \sigma, Q_{s} P_{s, a} \sigma^{\prime}\right\rangle_{s} .
$$

Thus from (4.17), we get

$$
\frac{\partial}{\partial s} \log \left(\left|\phi_{s}(\sigma)\right|_{\operatorname{det} H \bullet(X, F), s}^{L^{2}}\right)^{2}=-\operatorname{Tr}_{s}\left[Q_{s} P_{s, a}\right] .
$$

From (4.4), (4.15), and (4.18), we get (4.7).

4.3 Localization of the problem near the boundary $\boldsymbol{Y}$. We use freely the notation in subsections 1.2, 1.3, 1.5, 3.2. In the remainder of this section, we fix $s$ but most of the time we will omit the subscript $s$. 
In the sequel, if $\left(\beta_{u}\right)_{u>0}$ is a family of smooth forms (resp. differential operators) on $X$, we write $\beta_{u}=\mathcal{O}\left(u^{k}\right)$ as $u \rightarrow 0$ if for any $p \in \mathbb{N}$ there exists $C_{p}>0$ such that the $C^{p}$-norm of $\beta_{u}$ (resp. its coefficients) is dominated by $C_{p} u^{k}$.

Denote again by $\nabla_{s}^{T X}$ the Levi-Civita connection on $\left(T X, g_{s}^{T X}\right)$ with curvature $R_{s}^{T X}$, and let $\left\{e_{i}\right\}$ be an orthonormal basis of $\left(T X, g_{s}^{T X}\right)$. Set

$$
\begin{gathered}
\dot{g}_{s}^{T X}:=\left(g_{s}^{T X}\right)^{-1}\left(\frac{\partial}{\partial s} g_{s}^{T X}\right), \\
\dot{\omega}_{s}^{X}:=-\frac{1}{2} \sum_{1 \leq i, j \leq m}\left\langle\dot{g}_{s}^{T X} e_{i}, e_{j}\right\rangle_{g_{s}^{T X}} e^{i} \wedge \widehat{e^{j}} .
\end{gathered}
$$

By [BiZ1, Prop. 4.15], we have

$$
*_{s}^{-1} \frac{\partial *_{s}}{\partial s}=-\frac{1}{2} \sum_{1 \leq i, j \leq m}\left\langle e_{i}, \dot{g}_{s}^{T X} e_{j}\right\rangle_{g_{s}^{T X}} c\left(e_{i}\right) \widehat{c}\left(e_{j}\right) .
$$

Let $g_{s}^{T Y}$ be the metric on $T Y$ induced by $g_{s}^{T X}$. For $y \in Y$, let $\left\{e_{\alpha}\right\}_{\alpha=1}^{m-1}$ be an orthonormal basis of $\left(T_{y} Y, g_{s}^{T Y}\right)$. Let $\dot{\omega}_{s}^{Y}$ be the smooth section of $T^{*} Y \widehat{\otimes} \widehat{T^{*} Y}$ on $Y$ defined by

$$
\dot{\omega}_{s}^{Y}:=-\frac{1}{2} \sum_{1 \leq \alpha, \beta \leq m-1}\left\langle\dot{g}_{s}^{T X} e_{\alpha}, e_{\beta}\right\rangle_{g_{s}^{T X}} e^{\alpha} \wedge \widehat{e^{\beta}} .
$$

Theorem 4.6. We have the following identities in (4.6):

$$
\begin{aligned}
& M_{j, s}=0 \quad \text { if } \quad j \leq-2, \\
& M_{-1, s}=\operatorname{rk}(F) \int_{X} \int^{B_{X}} \dot{\omega}_{s}^{X} \exp \left(-\frac{1}{2} \dot{R}_{s}^{T X}\right) \\
& +\operatorname{rk}(F) \int_{Y} \int^{B_{Y}} \dot{\omega}_{s}^{Y} \exp \left(-\frac{1}{2}\left(\dot{R}_{s}^{T X} \mid Y\right)\right) \sum_{k=0}^{\infty} \frac{\dot{S}_{s}^{k}}{2 \Gamma\left(\frac{k}{2}+1\right)}, \\
& \lim _{t \rightarrow 0} \operatorname{Tr}_{s}\left[\left(h_{s}^{F}\right)^{-1} \frac{\partial h_{s}^{F}}{\partial s} e^{-t D_{a}^{2}}\right]=(-1)^{m} \int_{(X, Y)} \operatorname{Tr}\left[\left(h_{s}^{F}\right)^{-1} \frac{\partial h_{s}^{F}}{\partial s}\right] E\left(T X, \nabla_{s}^{T X}\right) .
\end{aligned}
$$

Proof. By [BiZ1, (4.61), (4.71), (4.73)] and [BGV, Thm 4.1], as $t \rightarrow 0$, uniformly on $X^{\prime}$, and implementing the notation introduced in subsection 1.3, we find

$$
\begin{aligned}
& \operatorname{Tr}_{s}\left[\left(h_{s}^{F}\right)^{-1} \frac{\partial h_{s}^{F}}{\partial s} e^{-t D^{\prime 2}}(x, x)\right] d v_{X^{\prime}}(x) \\
& \quad=\left(\operatorname{Tr}\left[\left(h_{s}^{F}\right)^{-1} \frac{\partial h_{s}^{F}}{\partial s}\right] \int^{B_{X}} \exp \left(-\frac{1}{2} \dot{R}_{s}^{T X}\right)\right)(x)+\mathcal{O}\left(t^{1 / 2}\right),
\end{aligned}
$$




$$
\begin{aligned}
& \operatorname{Tr}_{s}\left[*_{s}^{-1} \frac{\partial *_{s}}{\partial s} e^{-t D^{\prime 2}}(x, x)\right] d v_{X}(x) \\
& = \begin{cases}\left.\int^{B_{X}}\left\{\exp \left(-\frac{1}{2} \dot{R}_{s}^{T X}\right) \dot{\omega}_{s}^{X} \nabla_{s}^{T X} \theta \widehat{\left(F, h_{s}^{F}\right.}\right)\right\}(x)+\mathcal{O}(t) & \text { if } \operatorname{dim} X \text { is even } \\
\frac{1}{\sqrt{t}} \operatorname{rk}(F) \int^{B_{X}} \dot{\omega}_{s}^{X} \exp \left(-\frac{1}{2} \dot{R}_{s}^{T X}\right)(x)+\mathcal{O}\left(t^{\frac{1}{2}}\right) & \text { if } \operatorname{dim} X \text { is odd }\end{cases}
\end{aligned}
$$

where $\theta\left(F, h_{s}^{F}\right)$ is defined in (4.5) and the exponents of $t^{1 / 2}$ in (4.23b) are of the same parity as the dimension of $X$. Note that in [BiZ1, (4.71)], (4.23b) was worked out for $\operatorname{dim} X$ even. But proceeding as in [BiZ1, (4.55)-(4.63)], we get $(4.23 \mathrm{~b})$ for $\operatorname{dim} X$ odd, too.

We will use the notation of subsection 1.4 associated to the family of metrics $g_{s}^{T X}$, in the case $W=X$. In particular, we denote by $\widetilde{X}$ the fibers of the projection $\pi_{X \times \mathbb{R}}: X \times \mathbb{R} \rightarrow \mathbb{R}$, with boundary $\tilde{Y}$, and by $R^{T \tilde{X}}$ the curvature of $\nabla^{T \widetilde{X}}$. By [BiZ1, (4.74)-(4.85)], and (4.23b), and Stokes' theorem, we get

$$
\begin{aligned}
& \int_{X} \operatorname{Tr}_{s}\left[*_{s}^{-1} \frac{\partial *_{s}}{\partial s} e^{-t D^{\prime 2}}(x, x)\right] d v_{X}(x) \\
= & \frac{1}{\sqrt{t}} \operatorname{rk}(F) \int_{X} \int^{B_{X}} \dot{\omega}_{s}^{X} \exp \left(-\frac{1}{2} \dot{R}_{s}^{T X}\right)+\int_{X}\left[i\left(\frac{\partial}{\partial s}\right) e\left(T \widetilde{X}, \nabla^{T \widetilde{X}}\right)\right] \theta\left(F, h_{s}^{F}\right) \\
& \left.\quad \int_{Y} \int^{B_{X}} \theta \widehat{\left(F, h_{s}^{F}\right.}\right) \exp \left(-\frac{1}{2} \dot{R}_{s}^{T X}\right) \dot{\omega}_{s}^{X}+\mathcal{O}\left(t^{1 / 2}\right) .
\end{aligned}
$$

To get the boundary contribution, we proceed as in subsection 3.2. Arguing as in section 3, by (1.17), (3.11), we obtain as in (3.128), (3.133), (3.136),

$$
\begin{gathered}
d v_{Y}(y) \int_{0}^{t^{1 / 4}} \operatorname{Tr}_{s}\left[\left(h_{s}^{F}\right)^{-1} \frac{\partial h_{s}^{F}}{\partial s} P_{t}\left(\left(y, x_{m}\right),\left(y, x_{m}\right)\right)\right] \kappa\left(y, x_{m}\right) d v_{N}\left(x_{m}\right) \\
=d v_{Y}(y) \int_{0}^{\infty} 2^{m-1}(-1)^{\frac{m(m-1)}{2}} \operatorname{Tr}_{s}\left[\left(\left(h_{s}^{F}\right)^{-1} \frac{\partial h_{s}^{F}}{\partial s}\right)(y)\right. \\
\left.\quad\left[P_{0}^{(3)}\left(\left(0, x_{m}\right),\left(0, x_{m}\right)\right)\right]^{\max }\right] d v_{N}\left(x_{m}^{)}+\mathcal{O}\left(t^{1 / 4}\right)\right. \\
=(-1)^{m-1} \operatorname{Tr}\left[\left(h_{s}^{F}\right)^{-1} \frac{\partial h_{s}^{F}}{\partial s}(y)\right] e_{b}\left(Y, \nabla_{s}^{T X}\right)+\mathcal{O}\left(t^{1 / 4}\right) .
\end{gathered}
$$

By (0.2), (4.23a), and (4.25), as in (3.14),

$$
\begin{aligned}
\lim _{t \rightarrow 0} & \operatorname{Tr}_{s}\left[\left(h_{s}^{F}\right)^{-1} \frac{\partial h_{s}^{F}}{\partial s} e^{-t D_{a}^{2}}\right] \\
& =\lim _{t \rightarrow 0}\left\{\operatorname{Tr}_{s}\left[\left(h_{s}^{F}\right)^{-1} \frac{\partial h_{s}^{F}}{\partial s} e^{-t D^{\prime 2}}\right]+\operatorname{Tr}_{s}\left[\left(h_{s}^{F}\right)^{-1} \frac{\partial h_{s}^{F}}{\partial s} P_{t}\right]\right\} \\
& =(-1)^{m} \int_{(X, Y)} \operatorname{Tr}\left[\left(h_{s}^{F}\right)^{-1} \frac{\partial h_{s}^{F}}{\partial s}\right] E\left(T X, \nabla_{s}^{T X}\right) .
\end{aligned}
$$


For $y \in Y$, set

$$
\mathbb{I}_{t}(y)=\int_{0}^{t^{1 / 4}} \operatorname{Tr}_{s}\left[*_{s}^{-1} \frac{\partial *_{s}}{\partial s} P_{t}\left(\left(y, x_{m}\right),\left(y, x_{m}\right)\right)\right] \kappa\left(y, x_{m}\right) d v_{N}\left(x_{m}\right) .
$$

Then by (3.12),

$$
\operatorname{Tr}_{s}\left[*_{s}^{-1} \frac{\partial *_{s}}{\partial s}\left(e^{-t D_{a}^{2}}-e^{-t D^{\prime 2}}\right)\right]=\int_{Y} \mathbb{I}_{t}(y) d v_{Y}(y)+\mathcal{O}\left(e^{-c / t}\right) .
$$

But by (3.125), (4.20), as in (3.128), (3.133), and (3.136), we know that when $t \rightarrow 0$,

$$
\begin{aligned}
\lim _{t \rightarrow 0} \sqrt{t} & \mathbb{I}_{t}(y) d v_{Y}(y)=d v_{Y}(y) \int_{0}^{\infty} 2^{m-1}(-1)^{\frac{m(m-1)}{2}} \\
& \operatorname{Tr}_{F}\left[\left[\dot{\omega}_{s}^{Y}(y) P_{0}^{(3)}\left(\left(0, x_{m}\right),\left(0, x_{m}\right)\right)\right]^{\max }\right] d v_{N}\left(x_{m}\right) \\
= & \operatorname{rk}(F) \int^{B_{Y}} \dot{\omega}_{s}^{Y} \sum_{k=0}^{\infty} \frac{\dot{S}_{s}^{k}}{2 \Gamma\left(\frac{k}{2}+1\right)} \exp \left(-\frac{1}{2}\left(\dot{R}_{s}^{T X} \mid Y\right)\right) .
\end{aligned}
$$

By (4.6), (4.24), (4.26), (4.28), and (4.29), we get (4.22a).

By (4.22b), (4.24), and (4.28), it is now sufficient to calculate the asymptotics of $\mathbb{I}_{t}(y)$ when $t \rightarrow 0$.

4.4 Proof of Theorem 0.1: a special case. We use the same notation as in subsection 3.5. In this subsection, we will prove Theorem 0.1 under the additional assumption (4.30) below.

Let $g^{T X}$ be a metric on $T X$. We identify $Y \times[0, \varepsilon)$ with $\mathcal{U}_{\varepsilon}$ a neighborhood of $Y$ in $X$ as in subsection 1.2; and let $g_{0}^{T X}$ be a smooth metric on $T X$ verifying (1.22). In the rest of this section, we assume that the families $g_{s}^{T X}, h_{s}^{F}(s \in[0,1])$ featuring in the above analysis are defined by the equation (cf. (1.23))

$$
g_{s}^{T X}=s g^{T X}+(1-s) g_{0}^{T X}, \quad h_{s}^{F}=h^{F} .
$$

LEMMA 4.7 .

$$
\lim _{t \rightarrow 0} d v_{Y}(y) \mathbb{I}_{t}\left(y_{0}\right)=-\frac{2}{s} \operatorname{rk}(F) \int^{B_{Y}} \exp \left(-\frac{1}{2}\left(\dot{R}_{s}^{T X} \mid Y\right)_{y_{0}}\right) \sum_{k=1}^{\infty} \frac{\dot{S}_{s}^{k}}{4 \Gamma\left(\frac{k}{2}+1\right)} .
$$

Proof. For fixed $y_{0} \in Y$, we replace $X$ by $\mathbb{R}_{+}^{m} \hookrightarrow T_{y_{0}} X$ as in subsection 3.5, and we use the same trivialization of the vector bundle.

Let $M_{t}^{(2)}$ be obtained from $*_{s}^{-1} \frac{\partial *_{s}}{\partial s}$ by rescaling the coordinates as in (3.45) and $M_{t}^{(3)}$ be obtained from $M_{t}^{(2)}$ by replacing the Clifford variables $c\left(e_{\alpha}\right), \widehat{c}\left(e_{\alpha}\right)$ by $c_{t}\left(e_{\alpha}\right), \widehat{c}_{t}\left(\widehat{e_{\alpha}}\right)(1 \leq \alpha \leq m-1)$. Using Taylor expansion in 
$z_{m}$ near the boundary, we obtain from (1.7), (4.20), and (4.30)

$$
*_{s}^{-1} \frac{\partial *_{s}}{\partial s}=-\sum_{1 \leq i, j \leq m} \frac{1}{2}\left[\left(\mathcal{L}_{e_{m}} g^{T X}\right)\left(e_{i}, e_{j}\right) z_{m}+O\left(|Z|^{2}\right)\right] c\left(e_{i}\right) \widehat{c}\left(e_{j}\right) .
$$

By (1.10), (1.15), and (4.32), we know that

$$
M_{t}^{(3)}\left(0, z_{m}\right) \rightarrow M_{0}^{(3)}\left(0, z_{m}\right)=-2 \dot{S}_{1}\left(y_{0}\right) z_{m} .
$$

By (3.130), (3.131), (3.132), and (4.33), we have for $z_{m} \in \mathbb{R}_{+}$,

$$
\begin{array}{r}
\operatorname{Tr}_{s}\left[\left[\left(M_{0}^{(3)} P_{0}^{(3)}\right)\left(\left(0, z_{m}\right),\left(0, z_{m}\right)\right)\right]^{\max }\right] \\
=(4 \pi)^{-\frac{m-1}{2}} \operatorname{Tr}_{F}\left[\left[-2 \dot{S}_{1} z_{m}\left(Q_{4}-Q_{3}\right)\left(1,\left(0, z_{m}\right),\left(0, z_{m}\right)\right)\right.\right. \\
\left.\left.\exp \left(-\frac{1}{2}\left(\dot{R}_{s}^{T X} \mid Y\right)_{y_{0}}\right)\right]^{\max }\right] .
\end{array}
$$

By (3.31), (3.48), (3.49), and (3.117), we obtain

$$
\begin{aligned}
\lim _{t \rightarrow 0} \mathbb{I}_{t}\left(y_{0}\right)=\lim _{t \rightarrow 0} \int_{0}^{t^{1 / 4}} \operatorname{Tr}_{s}\left[*_{s}^{-1} \frac{\partial *_{s}}{\partial s} P_{t}^{1}\left(\left(0, z_{m}\right),\left(0, z_{m}\right)\right)\right] d v_{N}\left(z_{m}\right) \\
=\lim _{t \rightarrow 0} \int_{0}^{t^{1 / 4}} 2^{m-1}(-1)^{\frac{m(m-1)}{2}} \frac{1}{\sqrt{t}} \\
\quad \operatorname{Tr}_{s}\left[\left[\left(M_{t}^{(3)} P_{t}^{(3)}\right)\left(\left(0, \frac{z_{m}}{\sqrt{t}}\right),\left(0, \frac{z_{m}}{\sqrt{t}}\right)\right)\right]^{\max }\right] d v_{N}\left(z_{m}\right) \\
=\lim _{t \rightarrow 0} \int_{0}^{t^{-1 / 4}} 2^{m-1}(-1)^{\frac{m(m-1)}{2}} \\
\operatorname{Tr}_{s}\left[\left[\left(M_{t}^{(3)} P_{t}^{(3)}\right)\left(\left(0, z_{m}\right),\left(0, z_{m}\right)\right)\right]^{\max }\right] d v_{N}\left(z_{m}\right) \\
=\int_{0}^{\infty} 2^{m-1}(-1)^{\frac{m(m-1)}{2}} \operatorname{Tr}_{s}\left[\left[\left(M_{0}^{(3)} P_{0}^{(3)}\right)\left(\left(0, z_{m}\right),\left(0, z_{m}\right)\right)\right]^{\max }\right] d v_{N}\left(z_{m}\right) .
\end{aligned}
$$

Note that by (1.25), $\dot{S}_{s}=s \dot{S}_{1}$. By (1.1), (3.126), (3.135), (4.34), and (4.35), we get (4.31).

Proof of Theorem 0.1 under the assumption (4.30). In this case, $\left.\dot{\omega}_{s}^{X}\right|_{Y}=$ $\dot{\omega}_{s}^{Y}=0$. Now from (1.25), (1.39), (1.45), (4.7), (4.24), (4.28), and (4.31), we get

$\log \left(\left(\frac{\|\|_{\operatorname{det} H \bullet(X, F), 1}^{\mathrm{RS}}}{\|\|_{\operatorname{det} H \bullet(X, F), 0}^{\mathrm{RS}}}\right)^{2}\right)=\int_{X} \widetilde{e}\left(T X, \nabla_{s}^{T X}\right) \wedge \theta\left(F, h^{F}\right)+\operatorname{rk}(F) \int_{Y} B\left(\nabla^{T X}\right)$.

From (1.17), (1.25) and (1.39)

$$
i\left(\frac{\partial}{\partial s}\right) e_{b}\left(Y \times \mathbb{R}, \nabla^{T \widetilde{X}}\right)=0,
$$

which gives $\widetilde{e_{b}}\left(Y, \nabla_{s}^{T X}\right)=0$ by (1.45). Thus (4.36) implies Theorem 0.1. 
4.5 Examples. Let $f:[0, \varepsilon] \rightarrow \mathbb{R}^{+}$be a smooth function such that $f(0)=1$. We choose a family of metrics $g_{s}^{T X}$ on $T X$ such that on $Y \times[0, \varepsilon]$, $g_{0}^{T X}$ is defined by $(1.22)$, and for $s \in[0,1]$,

$$
g_{s}^{T X}\left(y, x_{m}\right)=f\left(s x_{\widetilde{m}}\right) g_{0}^{T X}, \quad h_{s}^{F}=h^{F} .
$$

As in subsection 4.3 , we denote by $\widetilde{X}$ the fibers of the projection $\pi_{X \times \mathbb{R}}$ : $X \times \mathbb{R} \rightarrow \mathbb{R}$. Then by (1.4), $\nabla_{\partial / \partial s}^{T \widetilde{X}} \frac{\partial}{\partial x_{m}}=0$ on $Y \times[0,1]$, thus by (1.10), as in (1.25), we get for $y_{0} \in Y, s \in[0,1], Z_{1}, Z_{2} \in T_{y_{0}} Y$,

$$
\begin{gathered}
\left\langle\widetilde{A}\left(y_{0}, s\right)\left(Z_{1}\right) Z_{2}, e_{m}\right\rangle=-\frac{s}{2} f^{\prime}(0)\left\langle Z_{1}, Z_{2}\right\rangle, \\
\dot{\widetilde{S}}\left(y_{0}, s\right)=\dot{S}_{s}\left(y_{0}\right)=s \dot{S}_{1}\left(y_{0}\right)=\frac{s}{4} f^{\prime}(0) \sum_{\alpha=1}^{m-1} e^{\alpha} \wedge \widehat{e^{\alpha}} .
\end{gathered}
$$

By (4.20), (4.38), and (4.39), the key equation (4.33) still holds. Now by the arguments as in (4.34), (4.35), we get again (4.31).

In the rest of this section, we suppose $F$ is the trivial bundle $\mathbb{C}$ on $X$. Let $\operatorname{Vol}(Y)$ be the Riemannian volume of $\left(Y, g^{T Y}\right)$. By (1.16), (4.7), (4.24), (4.28), (4.31), and (4.39), we get

$$
M_{0, s}=-\frac{2}{s} \int_{Y} \int^{B_{Y}} \exp \left(-\frac{1}{2} \dot{R}^{T Y}-s^{2} \dot{S}_{1}^{2}\right) \sum_{k=1}^{\infty} \frac{\left(s \dot{S}_{1}\right)^{k}}{4 \Gamma\left(\frac{k}{2}+1\right)} .
$$

By (1.1), and (4.39),

$$
\int^{B^{Y}} \dot{S}_{1}^{m-1}=\left(\frac{-1}{\sqrt{\pi}}\right)^{m-1}(m-1) !\left(\frac{f^{\prime}(0)}{4}\right)^{m-1} d v_{Y} .
$$

From (4.40) and (4.41), for $s \in[0,1], m=2,3$, we get

$M_{0, s}=-\frac{2}{s} \int_{Y} \int^{B^{Y}} \frac{\left(s \dot{S}_{1}\right)^{m-1}}{4 \Gamma((m+1) / 2)}=\frac{(-s)^{m-2}}{\pi}\left(\frac{f^{\prime}(0)}{4}\right)^{m-1} \operatorname{Vol}(Y)$.

Especially, if $\left(Y, g^{T Y}\right)$ is flat, then from (4.40) and (4.41), as in (1.19), if $m=2 k+1>1$ is odd, we have

$M_{0, s}=\frac{1}{2 s} \int_{Y} \int^{B^{Y}}\left(\exp \left(-s^{2} \dot{S}_{1}^{2}\right)-1\right)=\frac{(-1)^{k}(2 k) !}{2 \pi^{k} k !}\left(\frac{f^{\prime}(0)}{4}\right)^{2 k} s^{2 k-1} \operatorname{Vol}(Y)$.

If $f\left(x_{m}\right)=\left(1+x_{m}\right)^{m-1}, Y=\mathbb{T}^{m-1} \simeq \mathbb{R}^{m-1} / \mathbb{Z}^{m-1}$ is the flat torus with the metric $g^{T Y}$ induced by the canonical flat metric on $\mathbb{R}^{m-1}$ and $m=2,3$, then (4.42) recovers the results of [LüS, Appendix A] where Lück and Schick applied the explicit computation of the first few coefficients of the asymptotic expansion of the heat operator for manifolds with boundary by Branson and Gilkey [BrG]. (Note that $T_{a n}$ in [LüS, Def. 1.8] is $-\frac{\partial \theta^{F}}{\partial u}(0)$ in Definition 4.3. Moreover, in the computation of the mean curvature [LüS, p. 564, 1.-11], one missed a sign ' -'. Thus the last equality of line -7 therein should be corrected to ' $\cdots=(4 \pi)^{-1}(1+u) \neq 0$ '.) 


\section{Anomaly Formula: General Case}

The purpose of this section is to establish the anomaly formula for RaySinger metrics in the general case. We will introduce two Grassmann variables and use the techniques associated with the index theorem for families. In section 3, two copies of the bundle of Clifford algebras of $\left(T Y, g^{T Y}\right)$ appeared naturally, and the operators considered exhibited a symmetry property when these two copies were interchanged, which made the local index computations rather easy. But, generally speaking, this symmetry property will be broken in the local family index case, cf. [BiLo]. This is the reason why we need to introduce an unusual rescaling on these two Grassmann variables (cf. Remark 5.4) which does not work, however, for a general fibration.

This section is organized as follows. In subsection 5.1, we derive a Lichnerowicz type formula. In subsection 5.2, we explain why the problem is local near the boundary $Y$. In subsection 5.3, we explain the rescaling of the coordinate variable $Z$ and of the Clifford variable. In subsection 5.4, we calculate the heat kernel of the limit operator with the limit boundary condition. In subsection 5.5, we prove Theorem 0.1, finally, in subsection 5.6, we prove Theorem 4.2 .

We use the notation established in subsections 3.1, 4.1-4.3.

5.1 A Lichnerowicz formula. Let $X$ be a compact manifold with smooth boundary $\partial X=Y$ and $\operatorname{dim} X=m$. Let $F$ be a flat complex vector bundle on $X$ with flat connection $\nabla^{F}$. Let $\left(g_{s}^{T X}, h_{s}^{F}\right)_{s \in \mathbb{R}}$ be a smooth family of metrics on $T X, F$. We use the notation in subsection 3.1 with a subscript $s$. In the sequel, we fix $s$; if convenient, we will omit the subscript $s$.

We have already reduced the problem to finding the asymptotics of $\mathbf{I}_{t}=\operatorname{Tr}_{s}\left[*_{s}^{-1} \frac{\partial *_{s}}{\partial s} \exp \left(-t^{2} D_{s, a}^{2}\right)\right]$ when $t \rightarrow 0$ by $(4.22 \mathrm{~b})$. Hence in the following we may and will assume that $h_{s}^{F}=h^{F}$ for $s \in[0,1]$.

By (4.24), (4.27), (4.28) and (4.29), the asymptotic of $\mathbf{I}_{t}$ as $t \rightarrow 0$ has a singularity of order $1 / t$, but we do not know a direct way to compute its constant term. We know, however, that the leading term in the asymptotics of $\frac{\partial}{\partial t}\left(t \mathbf{I}_{t}\right)$ is of order $t^{0}$, hence, in this section, we will identify it with $\left[\operatorname{Tr}_{s}\left[e^{-\mathcal{L}_{t, a}^{(0)}}\right]\right]^{d \bar{s} d s}$ where $\mathcal{L}_{t, a}^{(0)}$ is a 2-order elliptic operator with the boundary condition (5.4). We need then the "strange" rescaling (5.2) in order to derive a "convergent" Lichnerowicz formula for the operator $\mathcal{L}_{t, a}^{(0)}$ as $t \rightarrow 0$, and then we proceed as in sections 3,4 , i.e. we separate the interior and 
boundary contribution. Again, our main achievement is to compute the boundary contribution (5.34).

Let $d \bar{s}$ be an odd Grassmann variable which anti-commutes with all odd elements in $\Lambda\left(T^{*} X\right)$ and $d s$. If $\beta \in \Lambda(\mathbb{C}(d \bar{s}, d s))$, let $[\beta]^{d \bar{s} d s} \in \mathbb{C}$ be the coefficient of $d \bar{s} d s$ in the expansion of $\beta$. If there exist $C_{1}, C_{2} \in \Lambda\left(T^{*} X\right)$, such that $\gamma=C_{1}+d s \wedge C_{2}$, then we write

$$
[\gamma]^{d s}=C_{2} .
$$

Now we introduce the scaling map

$$
\begin{aligned}
\psi_{t}: \Lambda(\mathbb{C}(d \bar{s}, d s)) \ni \beta_{0}+\beta_{10} d \bar{s}+\beta_{01} d s+\beta_{2} d \bar{s} d s & \mapsto \\
\beta_{0}+t^{-3 / 2} \beta_{10} d \bar{s}+t^{-1 / 2} \beta_{01} d s+t^{-2} \beta_{2} d \bar{s} d s & \in \Lambda(\mathbb{C}(d \bar{s}, d s)) ;
\end{aligned}
$$

then $\psi_{t}$ acts on $\Lambda(\mathbb{C}(d \bar{s}, d s)) \widehat{\otimes} \Lambda\left(T^{*} X\right)$ as $\psi_{t} \widehat{\otimes} 1$.

Let $\left\{e_{i}\right\}_{i=1}^{m}$ be an orthonormal basis of $\left(T X, g_{s}^{T X}\right)$. Let $d_{s}^{F *}$ be the formal adjoint of $d^{F}$ with respect to $g_{s}^{T X}, h^{F}$ as in subsection 3.1, and put

$$
B_{s}:=B=d_{s}^{F *}-d^{F}, \quad D_{1}^{2}=D^{2}-2 d \bar{s} \wedge B,
$$

$\mathcal{L}_{t}^{(0)}:=t^{2} D^{2}+\frac{1}{2} t^{3 / 2} d s \wedge\left[B, *_{s}^{-1} \frac{\partial *_{s}}{\partial s}\right]-2 t^{1 / 2} d \bar{s} \wedge B-d \bar{s} \wedge d s *_{s}^{-1} \frac{\partial *_{s}}{\partial s}$.

Then $\mathcal{L}_{t}^{(0)}$ is an operator acting on the smooth sections of $\mathcal{F}:=$ $\Lambda(\mathbb{C}(d s \oplus d \bar{s})) \widehat{\otimes} \Lambda\left(T^{*} X\right) \otimes F$ on $X$. We denote by $\mathcal{L}_{t, a}^{(0)}$ the operator associated to $\mathcal{L}_{t}^{(0)}$ and the following boundary condition

$$
\sigma \in C^{\infty}(X, \mathcal{F}) ; i\left(e_{\mathfrak{n}}\right) \sigma=i\left(e_{\mathfrak{n}}\right)\left(d^{F} \sigma-\frac{1}{2 \sqrt{t}} d s \wedge *_{s}^{-1} \frac{\partial *_{s}}{\partial s} \sigma\right)=0 \text { on } Y .
$$

Note that, by (3.4) and (5.3a), $B^{2}=-D^{2}$, and recall that ${ }^{s p} \nabla^{T X \otimes F}$, $\nabla^{T X \otimes F, u}$ are the connections on $\Lambda\left(T^{*} X\right) \otimes F$ induced by ${ }^{s p} \nabla^{T X}, \nabla^{F}$ and $\nabla^{T X}, \nabla^{F, u}$, as described in subsection 3.4. Since $\nabla^{T X}$ is torsion free, we obtain from (3.21), (3.26), (3.35), and (4.8) (cf. [BiLo, (3.42)]),

$$
B_{s}=\sum_{j=1}^{m}\left(-\widehat{c}\left(e_{j}\right) \nabla_{e_{j}}^{T X \otimes F, u}+\frac{1}{2} c\left(e_{j}\right) \omega\left(F, h^{F}\right)\left(e_{j}\right)\right) .
$$

Recall that $D_{a}$ was defined in (3.5). We introduce $B_{a}:=B \mid \mathcal{D}\left(D_{a}\right), D_{1, a}^{2}=$ $D_{1}^{2} \mid \mathcal{D}\left(D_{a}\right)$; then from (4.10), we get $B_{a}^{2}=-D_{a}^{2}$ because the boundary conditions are the same.

Lemma 5.1. For $\sigma \in \Omega(X, F)$, we have

$$
\left(e^{-t D_{a}^{2}} B \sigma\right)(x)=\left(B_{a} e^{-t D_{a}^{2}} \sigma\right)(x)+\int_{y \in Y} e^{-t D_{a}^{2}}(x, y) i\left(e_{\mathfrak{n}}\right) \sigma(y) d v_{Y}(y) .
$$


Proof. At first, by the identification of the orientation bundle $o(T X)$ and $o(T Y)$ in subsection 1.3, and (3.3), for $\sigma, \sigma^{\prime} \in \Omega(X, F)$,

$$
\begin{aligned}
\left\langle d^{F} \sigma, \sigma^{\prime}\right\rangle & =\left\langle\sigma, d^{F *} \sigma^{\prime}\right\rangle+\int_{Y}\left(\sigma \wedge *^{F} \sigma^{\prime}\right)_{F} \\
& =\left\langle\sigma, d^{F *} \sigma^{\prime}\right\rangle-\int_{Y}\left\langle e^{m} \wedge \sigma, \sigma^{\prime}\right\rangle(y) d v_{Y}(y), \\
\left\langle B \sigma, \sigma^{\prime}\right\rangle & =-\left\langle\sigma, B \sigma^{\prime}\right\rangle+\int_{Y}\left\langle\widehat{c}\left(e_{m}\right) \sigma, \sigma^{\prime}\right\rangle(y) d v_{Y}(y) .
\end{aligned}
$$

Thus for $\sigma \in \Omega(X, F), \sigma^{\prime} \in \mathcal{D}\left(D_{a}\right)$, by (5.7)

$$
\begin{aligned}
\left\langle B_{a} e^{-t D_{a}^{2}} \sigma, \sigma^{\prime}\right\rangle & =-\left\langle\sigma, e^{-t D_{a}^{2}} B_{a} \sigma^{\prime}\right\rangle=-\left\langle\sigma, B_{a} e^{-t D_{a}^{2}} \sigma^{\prime}\right\rangle \\
& =\left\langle B \sigma, e^{-t D_{a}^{2}} \sigma^{\prime}\right\rangle-\int_{y \in Y}\left\langle i\left(e_{\mathfrak{n}}\right) \sigma, e^{-t D_{a}^{2}} \sigma^{\prime}\right\rangle(y) d v_{Y}(y) \\
& =\left\langle e^{-t D_{a}^{2}} B \sigma, \sigma^{\prime}\right\rangle-\left\langle\int_{y \in Y} e^{-t D_{a}^{2}}(\cdot, y) i\left(e_{\mathfrak{n}}\right) \sigma(y) d v_{Y}(y), \sigma^{\prime}\right\rangle .
\end{aligned}
$$

From (5.8), we get (5.6).

\section{Theorem 5.2.}

$$
\frac{\partial}{\partial t}\left\{t \operatorname{Tr}_{s}\left[*_{s}^{-1} \frac{\partial *_{s}}{\partial s} e^{-t^{2} D_{a}^{2}}\right]\right\}=\operatorname{Tr}_{s}\left[e^{-\mathcal{L}_{t, a}^{(0)}}\right]^{d \bar{s} d s} .
$$

Proof. Let $p_{1}, p_{2}$ be the projections from $X \times X$ onto its first and second factors. By (3.5), (5.3b), and (5.4), there exists $\mathcal{P}_{t} \in \Lambda(\mathbb{C}(d \bar{s})) \widehat{\otimes} C^{\infty}(X \times X$, $\left.p_{1}^{*}\left(\Lambda\left(T^{*} X\right) \otimes F\right) \otimes p_{2}^{*}\left(\Lambda\left(T^{*} X\right) \otimes F\right)\right)$ such that

$$
e^{-t \mathcal{L}_{1, a}^{(0)}}=e^{-t D_{1, a}^{2}}+d s \wedge \mathcal{P}_{t} .
$$

Now, by (5.3b), and (5.4), $\mathcal{P}_{t}$ verifies the following equation,

$$
\left\{\begin{array}{l}
\left.i\left(e_{m}\right) \mathcal{P}_{t}\right|_{Y}=0 \\
\left.i\left(e_{m}\right)\left[d^{F}\left(d s \wedge \mathcal{P}_{t}\right)-\frac{1}{2} d s \wedge *_{s}^{-1} \frac{\partial *_{s}}{\partial s} e^{-t D_{1, a}^{2}}\right]\right|_{Y}=0 \\
\left(\frac{\partial}{\partial t}+D_{1}^{2}\right) d s \wedge \mathcal{P}_{t}+\left(\mathcal{L}_{1}^{(0)}-D_{1}^{2}\right) e^{-t D_{1, a}^{2}}=0
\end{array}\right.
$$

Thus from the proof of [RS, Lem.5.12, p.192-193], (5.11),

$$
\begin{gathered}
d s \wedge \mathcal{P}_{t}=-\int_{0}^{t} e^{-\left(t-t_{1}\right) D_{1, a}^{2}}\left(\mathcal{L}_{1}^{(0)}-D_{1, a}^{2}\right) e^{-t_{1} D_{1, a}^{2}} d t_{1}+\int_{0}^{t} d t_{1} \\
\int_{y \in Y} e^{-\left(t-t_{1}\right) D_{1, a}^{2}}(\cdot, y)\left(i\left(e_{\mathfrak{n}}\right)\left(-\frac{1}{2}\right) d s \wedge *_{s}^{-1} \frac{\partial *_{s}}{\partial s} e^{-t_{1} D_{1, a}^{2}}\right)(y, \cdot) d v_{Y}(y) .
\end{gathered}
$$

From the Volterra series expansion (cf. [BGV, Append. Ch. 9]) of $e^{-t D_{1, a}^{2}}$ and (5.6), we get

$$
e^{-t D_{1, a}^{2}}=e^{-t D_{a}^{2}}+2 t d \bar{s} \wedge B_{a} e^{-t D_{a}^{2}} .
$$


Thus from (5.6), (5.10), (5.12), and (5.13), we get

$$
\begin{aligned}
\operatorname{Tr}_{s}\left[e^{-t \mathcal{L}_{1, a}^{(0)}}\right]^{d \bar{s} d s}=-\operatorname{Tr}_{s}\left[t\left(\mathcal{L}_{1}^{(0)}-D_{1, a}^{2}\right) e^{-t D_{1, a}^{2}}\right]^{d \bar{s} d s} \\
\quad+t\left[\int_{y \in Y} \operatorname{Tr}_{s}\left[i\left(e_{\mathfrak{n}}\right)\left(-\frac{1}{2}\right) d s *_{s}^{-1} \frac{\partial *_{s}}{\partial s} e^{-t D_{1, a}^{2}}(y, y)\right] d v_{Y}(y)\right] d \bar{s} d s \\
=t \operatorname{Tr}_{s}\left[*_{s}^{-1} \frac{\partial *_{s}}{\partial s} e^{-t D_{a}^{2}}\right]-t^{2} \operatorname{Tr}_{s}\left[\left[B_{a}, *_{s}^{-1} \frac{\partial *_{s}}{\partial s}\right] B_{a} e^{-t D_{a}^{2}}\right] \\
\quad+t^{2} \int_{y \in Y} \operatorname{Tr}_{s}\left[i\left(e_{\mathfrak{n}}\right) *_{s}^{-1} \frac{\partial *_{s}}{\partial s} B_{a} e^{-t D_{a}^{2}}(y, y)\right] d v_{Y}(y) .
\end{aligned}
$$

Observe that $B_{a} e^{-t D_{a}^{2}}$ is a smooth operator, by (5.6),

$$
\begin{aligned}
\operatorname{Tr}_{s}[ & \left.*_{s}^{-1} \frac{\partial *_{s}}{\partial s} B_{a}^{2} e^{-t D_{a}^{2}}\right]=\operatorname{Tr}_{s}\left[*_{s}^{-1} \frac{\partial *_{s}}{\partial s} B_{a} e^{-\left(t-t_{1}\right) D_{a}^{2}} B_{a} e^{-t_{1} D_{a}^{2}}\right] \\
= & -\operatorname{Tr}_{s}\left[B_{a} e^{-t_{1} D_{a}^{2}} *_{s}^{-1} \frac{\partial *_{s}}{\partial s} B_{a} e^{-\left(t-t_{1}\right) D_{a}^{2}}\right] \\
= & -\operatorname{Tr}_{s}\left[e^{-t_{1} D_{a}^{2}} B *_{s}^{-1} \frac{\partial *_{s}}{\partial s} B_{a} e^{-\left(t-t_{1}\right) D_{a}^{2}}\right] \\
& +\operatorname{Tr}_{s}\left[\int_{y \in Y} e^{-t_{1} D_{a}^{2}}(\cdot, y)\left(i\left(e_{\mathfrak{n}}\right) *_{s}^{-1} \frac{\partial *_{s}}{\partial s} B_{a} e^{-t_{1} D_{a}^{2}}\right)(y, \cdot) d v_{Y}(y)\right] \\
= & -\operatorname{Tr}_{s}\left[B *_{s}^{-1} \frac{\partial *_{s}}{\partial s} B_{a} e^{-t D_{a}^{2}}\right] \\
& +\int_{y \in Y} \operatorname{Tr}_{s}\left[i\left(e_{\mathfrak{n}}\right) *_{s}^{-1} \frac{\partial *_{s}}{\partial s} B_{a} e^{-t D_{a}^{2}}(y, y)\right] d v_{Y}(y) .
\end{aligned}
$$

From (5.14), and (5.15), we get

$$
\begin{aligned}
\operatorname{Tr}_{s}\left[e^{-\mathcal{L}_{t, a}^{(0)}}\right]^{d \bar{s} d s}=t^{-2} \operatorname{Tr}_{s}\left[e^{-t^{2} \mathcal{L}_{1, a}^{(0)}}\right]^{d \bar{s} d s} & \\
& =\operatorname{Tr}_{s}\left[*_{s}^{-1} \frac{\partial *_{s}}{\partial s} e^{-t^{2} D_{a}^{2}}\right]+t^{2} \operatorname{Tr}_{s}\left[*_{s}^{-1} \frac{\partial *_{s}}{\partial s}\left(2 B_{a}^{2}\right) e^{-t^{2} D_{a}^{2}}\right] \\
& =\frac{\partial}{\partial t}\left\{t \operatorname{Tr}_{s}\left[*_{s}^{-1} \frac{\partial *_{s}}{\partial s} e^{-t^{2} D_{a}^{2}}\right]\right\} .
\end{aligned}
$$

Let $\nabla^{T X \otimes F, u} \omega\left(F, h^{F}\right)$ be the covariant derivative of $\omega\left(F, h^{F}\right)$, explicitly,

$$
\nabla_{e_{i}}^{T X \otimes F, u} \omega\left(F, h^{F}\right)\left(e_{j}\right)=\nabla_{e_{i}}^{F} \omega\left(F, h^{F}\right)\left(e_{j}\right)+\frac{1}{2}\left(\omega\left(F, h^{F}\right)\right)^{2}\left(e_{i}, e_{j}\right) .
$$

We will use the notation of subsections $1.4,1.5$ as in (4.24), especially, $\widetilde{j}: Y \times \mathbb{R} \rightarrow X \times \mathbb{R}$ is the natural embedding and $\widetilde{X}$ is the fibers of the projection $\pi_{X \times \mathbb{R}}: X \times \mathbb{R} \rightarrow \mathbb{R}$, with boundary $\tilde{Y}$. Then $\widetilde{j}^{*} R^{T \widetilde{X}} \in \Lambda^{2}\left(T^{*}(Y \times \mathbb{R})\right) \otimes \operatorname{End}(T X)$ is the restriction of $R^{T \widetilde{X}}$ to $Y \times \mathbb{R}$, and the forms $\dot{R}^{T \widetilde{X}}$ and $\dot{R}^{T \widetilde{X}} \mid \widetilde{Y}$ can be defined as in (1.13), (1.15). Let $\widetilde{A}, \dot{\widetilde{S}}$ be the tensors on $Y \times \mathbb{R}$ defined by (1.8) and (1.15) for the projection $\pi_{X \times \mathbb{R}}$. 
Then by (1.4), and (1.8), with $\dot{g}_{s}^{T X}$ from (4.19),

$$
\left\langle\widetilde{A}\left(\frac{\partial}{\partial s}\right) e_{\alpha}, e_{m}\right\rangle=\left\langle\nabla_{\partial / \partial s}^{T \widetilde{X}} e_{\alpha}, e_{m}\right\rangle=\frac{1}{2}\left\langle\dot{g}_{s}^{T X} e_{\alpha}, e_{m}\right\rangle_{g_{s}^{T X}} .
$$

Thus by (1.15), and (5.18),

$$
\dot{\widetilde{S}}=\dot{S}_{s}-\frac{1}{4} \sum_{\alpha=1}^{m-1}\left\langle\dot{g}_{s}^{T X} e_{\alpha}, e_{m}\right\rangle_{g_{s}^{T X}} d s \wedge \widehat{e^{\alpha}} .
$$

Our goal is a convergent Lichnerowicz formula for the operator $\mathcal{L}_{t}^{(0)}$, as $t \rightarrow 0$. To achieve this, we adopt [BiLo, (3.57)] to the fibration $\pi_{X \times \mathbb{R}}: X \times \mathbb{R} \rightarrow \mathbb{R}$ and introduce the connection $\nabla^{\mathcal{F}}$ on $\mathcal{F}$,

$$
\nabla^{\mathcal{F}}:=\nabla_{1}^{\mathcal{F}}:=\nabla^{T X \otimes F, u}-d \bar{s} \wedge \widehat{c}(\cdot)+\frac{1}{4} \sum_{j=1}^{m}\left\langle\dot{g}_{s}^{T X} \cdot, e_{j}\right\rangle_{g_{s}^{T X}} d s \wedge c\left(e_{j}\right),
$$

such that

$$
\begin{aligned}
\nabla_{t}^{\mathcal{F}}: & =\psi_{t} \nabla^{\mathcal{F}} \psi_{t}^{-1} \\
& =\nabla^{T X \otimes F, u}-\frac{1}{\sqrt{t^{3}}} d \bar{s} \wedge \widehat{c}(\cdot)+\frac{1}{4 \sqrt{t}} \sum_{j=1}^{m}\left\langle\dot{g}_{s}^{T X}, e_{j}\right\rangle_{g_{s}^{T X}} d s \wedge c\left(e_{j}\right)
\end{aligned}
$$

We denote by $\Delta_{t}^{\mathcal{F}}$ the connection Laplacian associated to $\nabla_{t}^{\mathcal{F}}$ as in (3.27). Then we have the following Lichnerowicz type formula,

Proposition 5.3. Let $\kappa$ be the scalar curvature of $\left(T X, g_{s}^{T X}\right)$. We have

$$
\begin{gathered}
\mathcal{L}_{t}^{(0)}=t^{2} \Delta_{t}^{\mathcal{F}}+\frac{t^{2} \kappa}{4}+\frac{t^{2}}{8} \sum_{i, j, k, l}\left\langle e_{k}, R^{T X}\left(e_{i}, e_{j}\right) e_{l}\right\rangle c\left(e_{i}\right) c\left(e_{j}\right) \widehat{c}\left(e_{k}\right) \widehat{c}\left(e_{l}\right) \\
+\frac{1}{8} t^{\frac{3}{2}} \sum_{i, j, k}\left[\left\langle e_{i},\left(\nabla_{e_{k}}^{T X} \dot{g}_{s}^{T X}\right) e_{j}\right\rangle_{g_{s}^{T X}}-\left\langle e_{i},\left(\nabla_{e_{j}}^{T X} \dot{g}_{s}^{T X}\right) e_{k}\right\rangle_{g_{s}^{T X}}\right] d s \wedge c\left(e_{i}\right) \widehat{c}\left(e_{j}\right) \widehat{c}\left(e_{k}\right) \\
+t^{2}\left(\frac{1}{4} \sum_{i}\left(\omega\left(F, h^{F}\right)\left(e_{i}\right)\right)^{2}-\frac{1}{8} \sum_{i, j}\left(c\left(e_{i}\right) c\left(e_{j}\right)-\widehat{c}\left(e_{i}\right) \widehat{c}\left(e_{j}\right)\right)\left(\omega\left(F, h^{F}\right)\right)^{2}\left(e_{i}, e_{j}\right)\right. \\
\left.-\frac{1}{2} \sum_{i, j} c\left(e_{i}\right) \widehat{c}\left(e_{j}\right)\left(\nabla_{e_{i}}^{T X \otimes F, u} \omega\left(F, h^{F}\right)\right)\left(e_{j}\right)\right) \\
+\frac{1}{4} t^{\frac{3}{2}} \sum_{i, j}\left\langle\dot{g}_{s}^{T X} e_{i}, e_{j}\right\rangle_{g_{s}^{T X}} \omega\left(F, h^{F}\right)\left(e_{j}\right) d s \wedge \widehat{c}\left(e_{i}\right)-t^{\frac{1}{2}} \sum_{i} \omega\left(F, h^{F}\right)\left(e_{i}\right) d \bar{s} \wedge c\left(e_{i}\right) .
\end{gathered}
$$

Proof. If $d s=d \bar{s}=0,(5.22)$ is exactly (3.28). By using (3.22), (4.20) and (5.5), we get

$$
\begin{aligned}
\frac{1}{2}\left[B, *_{s}^{-1} \frac{\partial *_{s}}{\partial s}\right]= & \sum_{i, j}\left(\frac{1}{4}\left\langle\dot{g}_{s}^{T X} e_{i}, e_{j}\right\rangle \omega\left(F, h^{F}\right)\left(e_{j}\right) \widehat{c}\left(e_{i}\right)\right. \\
& -\frac{1}{4} \sum_{k}\left\langle e_{i},\left(\nabla_{e_{j}}^{T X} \dot{g}_{s}^{T X}\right) e_{k}\right\rangle c\left(e_{i}\right) \widehat{c}\left(e_{j}\right) \widehat{c}\left(e_{k}\right)
\end{aligned}
$$




$$
\left.-\frac{1}{2}\left\langle\dot{g}_{s}^{T X} e_{i}, e_{j}\right\rangle c\left(e_{i}\right) \nabla_{e_{j}}^{T X \otimes F, u}\right) .
$$

Thus we obtain the additional contributions from $d s$ and $d \bar{s}$.

REMARK 5.4. The above proposition is a variant of [BiLo, Th. 2.11] for the fibration $\pi_{X \times \mathbb{R}}: X \times \mathbb{R} \rightarrow \mathbb{R}$. We use a different rescaling for the variables $d s, d \bar{s}$, however, which is crucial here. Also, we get from [BiZ1, (4.79)-(4.81)] (or by comparing (5.22) and [BiLo, (3.58)]),

$$
\left\langle e_{j}, R^{T \widetilde{X}}\left(\frac{\partial}{\partial s}, e_{i}\right) e_{k}\right\rangle=\frac{1}{2}\left\{\left\langle e_{i},\left(\nabla_{e_{k}}^{T X} \dot{g}_{s}^{T X}\right) e_{j}\right\rangle-\left\langle e_{i},\left(\nabla_{e_{j}}^{T X} \dot{g}_{s}^{T X}\right) e_{k}\right\rangle\right\} .
$$

5.2 Localization of the problem near the boundary $Y$. As in subsection 3.2, we embed the manifold $X$ into a closed manifold $X^{\prime}$ and extend everything smoothly to $X^{\prime}$; then we denote by $e^{-u^{2} \mathcal{L}_{t, a}^{(0)}}\left(x, x^{\prime}\right)$ and $e^{-u^{2} \mathcal{L}_{t}^{(0)}}\left(x, x^{\prime}\right)$ the kernel of $e^{-u^{2} \mathcal{L}_{t, a}^{(0)}}$ and $e^{-u^{2} \mathcal{L}_{t}^{(0)}}$ on $X$ and $X^{\prime}$, with respect to $d v_{X^{\prime}}\left(x^{\prime}\right)$, respectively.

Again by [RS, Lem. 5.12] there exist $c, C>0$ such that for $x, x^{\prime} \in X$, $t \in(0,1]$

$$
\left|\left(e^{-t^{2} \mathcal{L}_{1}^{(0)}}-e^{-t^{2} \mathcal{L}_{1, a}^{(0)}}\right)\left(x, x^{\prime}\right)\right| \leq \frac{C}{t^{m}} e^{-\frac{c}{t^{2}}\left(d^{2}(x, Y)+d^{2}\left(x^{\prime}, Y\right)+d^{2}\left(x, x^{\prime}\right)\right)} .
$$

Note that $\mathcal{L}_{t}^{(0)}=\psi_{t} t^{2} \mathcal{L}_{1}^{(0)} \psi_{t}^{-1}$, thus by replacing $d s$ by $\frac{1}{\sqrt{t}} d s, d \bar{s}$ by $\frac{1}{\sqrt{t^{3}}} d \bar{s}$ in $e^{-t^{2} \mathcal{L}_{1, a}^{(0)}}\left(x, x^{\prime}\right)$, we obtain $e^{-\mathcal{L}_{t, a}^{(0)}}\left(x, x^{\prime}\right)$. Thus

$$
\left|\left(e^{-\mathcal{L}_{t}^{(0)}}-e^{-\mathcal{L}_{t, a}^{(0)}}\right)\left(x, x^{\prime}\right)\right| \leq \frac{C}{t^{m+2}} e^{-\frac{c}{t^{2}}\left(d^{2}(x, Y)+d^{2}\left(x^{\prime}, Y\right)+d^{2}\left(x, x^{\prime}\right)\right)} .
$$

By (3.23), (5.20), and (5.24), the curvature of $\nabla^{\mathcal{F}}$ is given by

$$
\begin{aligned}
& \left(\nabla^{\mathcal{F}}\right)^{2}\left(e_{i}, e_{j}\right)=\frac{1}{4} \sum_{k, l}\left\langle e_{l}, R^{T X}\left(e_{i}, e_{j}\right) e_{k}\right\rangle\left(c\left(e_{k}\right) c\left(e_{l}\right)-\widehat{c}\left(e_{k}\right) \widehat{c}\left(e_{l}\right)\right) \\
& -\frac{1}{4} \omega^{2}\left(F, h^{F}\right)\left(e_{i}, e_{j}\right)-\frac{1}{2} \sum_{k}\left\langle e_{i}, R^{T \widetilde{X}}\left(\frac{\partial}{\partial s}, e_{k}\right) e_{j}\right\rangle_{g_{s}^{T X}} d s \wedge c\left(e_{k}\right) .
\end{aligned}
$$

From (3.22) and (5.20), for smooth sections $U, V$ of $T X$ over $X$, we have

$$
\begin{aligned}
& \nabla_{U}^{\mathcal{F}} c(V)=c\left(\nabla_{U}^{T X} V\right)-\frac{1}{2} d s \wedge\left\langle\dot{g}_{s}^{T X} U, V\right\rangle_{g_{s}^{T X}}, \\
& \nabla_{U}^{\mathcal{F}} \widehat{c}(V)=\widehat{c}\left(\nabla_{U}^{T X} V\right)-2 d \bar{s} \wedge\langle U, V\rangle_{g_{s}^{T X}}
\end{aligned}
$$

Now fix $x_{0} \in X^{\prime}$ and a normal coordinate system in $X^{\prime}$ centered at $x_{0}$, let $\left\{\widetilde{e}_{i}(Z)\right\}$ for $Z \in T_{x_{0}} X^{\prime}$ be the orthogonal basis of $T_{Z} X^{\prime}$ obtained by parallel transport of the orthogonal basis $\left\{e_{i}\right\}$ of $T_{x_{0}} X^{\prime}$ with respect to $\nabla^{T X^{\prime}}$ along the geodesic $u \rightarrow u Z$. We identify $\mathcal{F}_{Z}$ with $\mathcal{F}_{x_{0}}$ by parallel transport with respect to $\nabla^{\mathcal{F}}$ along the curve $u \rightarrow u Z$, then under this identification, by (5.28), 


$$
\begin{aligned}
& c\left(\widetilde{e}_{i}(Z)\right)=c\left(e_{i}\right)-\frac{1}{2}\left(\left\langle\dot{g}_{s}^{T X} Z, e_{i}\right\rangle_{g_{s}^{T X}}+\mathcal{O}\left(|Z|^{2}\right)\right) d s \wedge, \\
& \widehat{c}\left(\widetilde{e}_{i}(Z)\right)=\widehat{c}\left(e_{i}\right)-\left(2 z_{i}+\mathcal{O}\left(|Z|^{2}\right)\right) d \bar{s} .
\end{aligned}
$$

We now introduce a rescaling $\widetilde{\psi}_{t}$ in sending $z \mapsto t z, \nabla_{e_{j}} \mapsto t^{-1} \nabla_{e_{j}}, c\left(e_{j}\right) \mapsto$ $c_{t^{2}}\left(e_{j}\right), \widehat{c}\left(e_{j}\right) \mapsto \widehat{c}_{t^{2}}\left(\widehat{e_{j}}\right), d s \mapsto t^{-1 / 2} d s, d \bar{s} \mapsto t^{-3 / 2} d \bar{s}$. By using (5.22), (5.27), and (5.29), we find that as $t \rightarrow 0$, the rescaled operator $\mathcal{L}_{t}:=$ $\widetilde{\psi}_{t} t^{2} \mathcal{L}_{1}^{(0)} \widetilde{\psi}_{t}^{-1}$ converges to

$\mathcal{L}_{0}=\Delta^{T_{x} X}+\frac{1}{4} \sum_{i, j}\left\langle e_{i}, R_{x_{0}}^{T \tilde{X}} e_{j}\right\rangle\left(\widehat{e^{i}}-2 z_{i} d \bar{s}\right) \wedge\left(\widehat{e^{j}}-2 z_{j} d \bar{s}\right)-d \bar{s} \wedge \omega\left(F, h^{F}\right)_{x_{0}}$,

in the sense that $\mathcal{L}_{t} \sigma \rightarrow \mathcal{L}_{0} \sigma$ for any smooth section $\sigma$ with compact support.

Let $e^{-\mathcal{L}_{0}}\left(x, x^{\prime}\right)\left(x, x^{\prime} \in T_{x_{0}} X^{\prime}\right)$ be the smooth kernel of $e^{-\mathcal{L}_{0}}$ calculated with respect to $d v_{T_{x_{0}} X^{\prime}}\left(x^{\prime}\right)$. By (5.30) and the Volterra series expansion (cf. [BGV, Append., Ch. 9]) of $e^{-\mathcal{L}_{0}}$, we find

$$
e^{-\mathcal{L}_{0}}(0,0)=(4 \pi)^{-m / 2} \exp \left(-\frac{1}{2} \dot{R}_{x_{0}}^{T \tilde{X}}+d \bar{s} \wedge \omega\left(F, h^{F}\right)_{x_{0}}\right) .
$$

Thus by (5.30), (5.31), and a standard argument in local index theory [BiLo, §3], [BiZ1, §4] we see that uniformly on $x \in X^{\prime}$,

$$
\begin{array}{r}
\lim _{t \rightarrow 0} \operatorname{Tr}_{s}\left[e^{-\mathcal{L}_{t}^{(0)}}(x, x)\right]^{d \bar{s} d s} d v_{X}(x)=2^{m}(-1)^{m(m+1) / 2}(4 \pi)^{-m / 2} \\
\operatorname{Tr}_{F}\left[\left[\exp \left(-\frac{1}{2} \dot{R}^{T \widetilde{X}}+d \bar{s} \wedge \omega\left(F, h^{F}\right)\right)\right]^{\operatorname{Max}}\right]^{d \bar{s} d s} d v_{X}(x) \\
=\int^{B_{X}}\left\{\exp \left(-\frac{1}{2} \dot{R}^{T \widetilde{X}}\right) \theta\left(F, h^{F}\right)\right\}^{d s} .
\end{array}
$$

Here we denote by [ ] $]^{\text {Max }}$ the coefficient of $e^{1} \wedge \cdots \wedge e^{m} \wedge \widehat{e^{1}} \cdots \wedge \widehat{e^{m}}$. Hence by $(5.32)$,

$$
\int_{X} \operatorname{Tr}_{s}\left[e^{-\mathcal{L}_{t}^{(0)}}(x, x)\right]^{d \bar{s} d s} d v_{X}(x)=\int_{X}\left[e\left(T \widetilde{X}, \nabla^{T \widetilde{X}}\right)\right]^{d s} \theta\left(F, h^{F}\right)+\mathcal{O}\left(t^{1 / 2}\right) .
$$

By (5.26), (5.33), thus it remains to determine, when $t \rightarrow 0$, the asymptotics of

$$
\mathcal{J}_{t}(y)=\int_{0}^{t^{1 / 4}} \operatorname{Tr}_{s}\left[\left[\left(e^{-\mathcal{L}_{t, a}^{(0)}}-e^{-\mathcal{L}_{t}^{(0)}}\right)\left(\left(y, x_{m}\right),\left(y, x_{m}\right)\right)\right]^{d \bar{s} d s}\right] \kappa\left(y, x_{m}\right) d v_{N}\left(x_{m}\right) .
$$


Comparing with (4.27), we see that the constant terms in the asymptotics of $\mathcal{J}_{t}(y)$ and $\mathbb{I}_{t}(y)$, when $t \rightarrow 0$, differ by the last term in (4.24). But $\mathbb{I}_{t}(y)$ has a singular term (cf. (4.29)) when $t \rightarrow 0$, thus we cannot compute directly its constant term. Here we will prove in fact that $\lim _{t \rightarrow 0} \mathcal{J}_{t}(y)$ exists.

5.3 Rescaling. We use the notation introduced in subsection 3.5.

To calculate $\lim _{t \rightarrow 0} \mathcal{J}_{t}(y)$, we will first localize the problem on $Y$. Then in view of (3.31), we transfer the problem for operators acting on $C^{\infty}\left(\mathbb{R}_{+}^{m}, \mathcal{F}\right)=C^{\infty}\left(\mathbb{R}_{+}^{m}, \Lambda(\mathbb{C}(d \bar{s}, d s)) \widehat{\otimes} \Lambda\left(T^{*} X\right) \otimes F\right)$ to a problem for operators acting on

$$
C^{\infty}\left(\mathbb{R}_{+}^{m}, \Lambda(\mathbb{C}(d \bar{s}, d s)) \widehat{\otimes}\left(\Lambda\left(T^{*} Y\right) \widehat{\otimes} \widehat{\Lambda\left(T^{*} Y\right)} \widehat{\otimes} \Lambda\left(N^{*}\right) \otimes F\right)_{y_{0}}\right),
$$

by an appropriate rescaling.

Let $P^{1} T X, P^{2} T X$ be the orthogonal projections from $T X={ }^{1} T X \oplus^{2} T X$ onto ${ }^{1} T X,{ }^{2} T X$ as in subsection 1.2. Let $\left\{e_{\alpha}\right\}_{\alpha=1}^{m-1}$ be an orthonormal basis of ${ }^{1} T X$.

Let ${ }^{s p} \nabla^{\mathcal{F}, A}$ be the connection on $\mathcal{F}$ on $Y \times[0, \varepsilon) \subset X$,

$$
\begin{aligned}
{ }^{s p} \nabla^{\mathcal{F}, A}={ }^{s p} \nabla^{T X \otimes F} & -d \bar{s} \wedge \widehat{c}(\cdot)+\frac{1}{4} \sum_{\beta=1}^{m-1}\left\langle\dot{g}_{s}^{T X} \cdot, e_{\beta}\right\rangle d s \wedge c\left(e_{\beta}\right) \\
& +A^{\Lambda\left(T^{*} Y\right)}-\frac{1}{4} \sum_{\beta=1}^{m-1}\left\langle\dot{g}_{s}^{T X} P^{2} T X, e_{\beta}\right\rangle d s \wedge \widehat{c}\left(e_{\beta}\right) .
\end{aligned}
$$

As ${ }^{s p} \nabla^{T X}$ is a metric connection, from (3.22) and (5.35), as in (3.61), if $U, V$ are smooth sections of $T X$ over $Y \times[0, \varepsilon)$, then

$$
\begin{aligned}
& {\left[{ }^{s p} \nabla_{U}^{\mathcal{F}, A}, c(V)\right]=c\left({ }^{s p} \nabla_{U}^{T X} V\right)-\sum_{\beta=1}^{m-1}\left\langle P^{2} T X \quad U, A\left(P^{1} T X V\right) e_{\beta}\right\rangle \widehat{c}\left(e_{\beta}\right)} \\
& -\frac{1}{2}\left\langle\dot{g}_{s}^{T X} U, P^{1} T X V\right\rangle_{g_{s}^{T X}} d s, \\
& {\left[{ }^{s p} \nabla_{U}^{\mathcal{F}, A}, \widehat{c}(V)\right]=\widehat{c}\left({ }^{s p} \nabla_{U}^{T X} V\right)-\sum_{\beta=1}^{m-1}\left\langle P^{2} T X U, A\left(e_{\beta}\right) P^{1} T X V\right\rangle c\left(e_{\beta}\right)} \\
& -2 d \bar{s} \wedge\langle U, V\rangle_{g_{s}^{T X}}-\frac{1}{2}\left\langle\dot{g}_{s}^{T X} P^{2} T X U, P^{1 T X} V\right\rangle d s .
\end{aligned}
$$

Let $T^{X}$ be the torsion of ${ }^{s p} \nabla^{T X}$ along the fiber $X$. Then by (3.23) and (5.24), the curvature ${ }^{s p} R^{\mathcal{F}, A}$ of ${ }^{s p} \nabla^{\mathcal{F}, A}$ on $Y \times[0, \varepsilon)$ is given by 


$$
\begin{aligned}
& { }^{s p} R^{\mathcal{F}, A}\left(e_{i}, e_{\alpha}\right)=\frac{1}{4} \sum_{k, l}\left\langle e_{l}{ }^{s p} R^{T X}\left(e_{i}, e_{\alpha}\right) e_{k}\right\rangle\left(c\left(e_{k}\right) c\left(e_{l}\right)-\widehat{c}\left(e_{k}\right) \widehat{c}\left(e_{l}\right)\right) \\
& +\frac{1}{4} \sum_{\beta}\left\langle e_{\beta},\left({ }^{s p} \nabla_{e_{i}}^{T X} \dot{g}_{s}^{T X}\right) e_{\alpha}-\left({ }^{s p} \nabla_{e_{\alpha}}^{T X} \dot{g}_{s}^{T X}\right) e_{i}+\dot{g}_{s}^{T X} T^{X}\left(e_{i}, e_{\alpha}\right)\right\rangle d s \wedge c\left(e_{\beta}\right) \\
& -d \bar{s} \wedge \widehat{c}\left(T^{X}\left(e_{i}, e_{\alpha}\right)\right)+\frac{1}{2} \sum_{\beta, \gamma=1}^{m-1}\left\langle\left({ }^{s p} \nabla_{e_{\alpha}}^{T X} A^{T Y}\right)\left(P^{2} T X e_{i}\right) e_{\beta}, e_{\gamma}\right\rangle c\left(e_{\beta}\right) \widehat{c}\left(e_{\gamma}\right) \\
& +\frac{1}{2} \sum_{\beta=1}^{m-1}\left\langle\left({ }^{s p} \nabla_{e_{\alpha}}^{T X} A^{T Y}\right)\left(P^{2} T X e_{i}\right) e_{\beta}, e_{\beta}\right\rangle+\sum_{\beta=1}^{m-1}\left\langle A\left(e_{\beta}\right) e_{\alpha}, P^{2} T X e_{i}\right\rangle d \bar{s} \wedge c\left(e_{\beta}\right) \\
& \quad-\frac{1}{4} \sum_{\beta, \gamma=1}^{m-1}\left\langle\dot{g}_{s}^{T X} e_{\alpha}, e_{\gamma}\right\rangle\left\langle A\left(e_{\gamma}\right) e_{\beta}, P^{2} T X e_{i}\right\rangle d s \wedge \widehat{c}\left(e_{\beta}\right) \\
& +\frac{1}{4} \sum_{\beta=1}^{m-1}\left\langle\left({ }^{s p} \nabla_{e_{\alpha}}^{T X} \dot{g}_{s}^{T X}\right) P^{2} T X e_{i}, e_{\beta}\right\rangle d s \wedge \widehat{c}\left(e_{\beta}\right)+\frac{1}{2}\left\langle\dot{g}_{s}^{T X} P^{2} T X e_{i}, e_{\alpha}\right\rangle d \bar{s} \wedge d s .
\end{aligned}
$$

By Proposition 3.7, (4.20), and (5.35), the boundary condition (5.4) is equivalent to

$$
\left\{\begin{array}{l}
\left.i\left(e_{m}\right) \sigma\right|_{Y}=0, \\
\left.w\left(e^{m}\right) \psi_{t}^{s p} \nabla_{e_{m}}^{\mathcal{F}, A} \psi_{t}^{-1} \sigma\right|_{Y}=0 .
\end{array}\right.
$$

Choose $y_{0} \in Y=\partial X$. We replace $X$ by a half-space in $T_{y_{0}} X$, and trivialize $T X$ exactly as in subsection 3.5. Let $\left\{e_{\alpha}\right\}_{\alpha=1}^{m-1}$ be an orthonormal basis of $T_{y_{0}} Y$, and let $\left\{\widetilde{e}_{\alpha}(Z)\right\}_{\alpha=1}^{m-1}$ (resp. $e_{m}$ ) be the orthonormal basis of ${ }^{1} T X_{Z},{ }^{2} T X_{Z}$ defined in subsection 3.5.

For $Z=\left(Z^{\prime}, Z_{m}\right) \in T_{y_{0}} X$, we identify $\mathcal{F}_{Z}$ to $\mathcal{F}_{y_{0}}$ by parallel transport with respect to the connection $\psi_{t}^{s p} \nabla^{\mathcal{F}, A} \psi_{t}^{-1}$ (cf. (5.2)) along the curve $\gamma_{Z}$ : $[0,1] \ni u \mapsto u Z^{\prime},[1,2] \ni u \mapsto Z^{\prime}+(u-1) Z_{m}$. The above trivialization induces an invertible linear map

$$
\Phi: C^{\infty}\left(B_{\epsilon}^{Y}\left(y_{0}\right) \times[-\epsilon, \epsilon], \mathcal{F}\right) \rightarrow C^{\infty}\left(B_{\epsilon}^{T_{y} Y}(0) \times[-\epsilon, \epsilon], \mathcal{F}_{y_{0}}\right) .
$$

For any operator $C$ on $C^{\infty}\left(B_{\epsilon}^{Y}\left(y_{0}\right) \times[-\epsilon, \epsilon], \mathcal{F}\right)$, we write $C_{\Phi}:=\Phi C \Phi^{-1}$.

With the function $\varphi$ defined in (3.39), we put

$$
\mathcal{L}_{t}^{(1)}:=\varphi^{2}(|Z|)\left(\mathcal{L}_{t}^{(0)}\right)_{\Phi}+\left(1-\varphi^{2}(|Z|)\right)\left(t^{2} \Delta^{T_{y_{0}} X}\right) .
$$

which we regard as an operator on $\mathbb{R}^{m}$. For $t>0, \sigma \in C^{\infty}\left(\mathbb{R}^{m}, \mathcal{F}_{y_{0}}\right)$, set

$$
\left(G_{t} \sigma\right)(z):=\sigma(z / t), \quad \mathcal{L}_{t}^{(2)}:=G_{t}^{-1} \mathcal{L}_{t}^{(1)} G_{t} .
$$

Then $\mathcal{L}_{t}^{(2)}$ is a differential operator on $\mathbb{R}^{m}$ with coefficients in $\operatorname{End}(\mathcal{F})_{y_{0}}$. Next we enlarge the coefficient space as common in local index theory, and 
replace $\mathcal{F}_{y_{0}}$ by

$$
\left.\mathcal{K}_{y_{0}}:=\Lambda(\mathbb{C}(d \bar{s}, d s)) \widehat{\otimes}\left(\Lambda\left(T^{*} Y\right) \widehat{\otimes} \widehat{\Lambda\left(T^{*} Y\right.}\right) \widehat{\otimes} \Lambda\left(N^{*}\right) \otimes F\right)_{y_{0}} .
$$

Then $\mathcal{L}_{t}^{(2)}$ induces a differential operator $\mathcal{L}_{t}^{(3)}$ with coefficients in $\operatorname{End}\left(\mathcal{K}_{y_{0}}\right)$ by replacing the Clifford variables $c\left(e_{\alpha}\right)$ and $\widehat{c}\left(e_{\alpha}\right)$ by $c_{t^{2}}\left(e_{\alpha}\right)$ and $\widehat{c}_{t^{2}}\left(\widehat{e_{\alpha}}\right)$ $(1 \leq \alpha \leq m-1)$ (cf. (3.21) and (3.30)). We denote by $\mathcal{L}_{t, a}^{(1)}$ the operator $\mathcal{L}_{t}^{(1)}$ with the boundary condition

$\left\{\begin{array}{l}\left.\left\{\varphi^{2}(|z|)\left(i\left(e_{m}(z)\right)\right)_{\Phi}+\left(1-\varphi^{2}(|z|)\right) i\left(e_{m}\right)\right\} \sigma\right|_{z_{m}=0}=0, \\ \left.\left\{\varphi^{2}(|z|)\left(w\left(e^{m}(z)\right) \psi_{t}{ }^{s p} \nabla_{e_{m}}^{\mathcal{F}, A} \psi_{t}^{-1}\right)_{\Phi}+\left(1-\varphi^{2}(|z|)\right) w\left(e^{m}\right) \nabla_{e_{m}}\right\} \sigma\right|_{z_{m}=0}=0 .\end{array}\right.$

From (5.36), we derive the identities for $z \in \mathbb{R}^{m}, z^{\prime} \in \mathbb{R}^{m-1}$

$$
\begin{aligned}
& \left(c\left(e_{m}\right)\right)_{\Phi}(z)=c\left(e_{m}\right), \quad\left(\psi_{t}^{s p} \nabla_{e_{m}}^{\mathcal{F}, A} \psi_{t}^{-1}\right)_{\Phi, z}=\nabla_{e_{m}}, \\
& \left(\widehat{c}\left(e_{m}\right)\right)_{\Phi}\left(z^{\prime}\right)=\widehat{c}\left(e_{m}\right) .
\end{aligned}
$$

By (5.43), the boundary condition (5.42) is equivalent to

$$
\left\{\begin{array}{l}
\left.i\left(e_{m}\right) \sigma\right|_{z_{m}=0}=0, \\
\left.\nabla_{e_{m}} w\left(e^{m}\right) \sigma\right|_{z_{m}=0}=0,
\end{array}\right.
$$

which is invariant under rescaling. We denote by $\mathcal{L}_{t, a}^{(i)}$ the operator $\mathcal{L}_{t}^{(i)}$ with the boundary condition (5.44).

If $C$ is any differential operator, we denote by $C_{t}^{(3)}$ the operator obtained from $C$ by rescaling the coordinate $z$ and the Clifford variable as above.

Recall that $A$ is defined in (1.8), for $z \in \mathbb{R}^{m}$, set

$$
\begin{aligned}
& A_{\alpha \beta}=\left\langle A_{s, y_{0}}\left(e_{\alpha}\right) e_{\beta}, e_{m}\right\rangle_{g_{s}^{T X}}, \\
& \mathcal{A}_{\alpha}=\sum_{\beta=1}^{m-1} A_{\alpha \beta} e^{\beta}+\frac{1}{2}\left\langle\dot{g}_{s, y_{0}}^{T X} e_{m}, e_{\alpha}\right\rangle d s, \\
& \Theta(z)=\sum_{\substack{1 \leq \alpha \leq m-1 \\
1 \leq i \leq m}}\left\langle e_{\alpha},\left(\widetilde{j}^{*} R^{T \widetilde{X}}\right)_{y_{0}} e_{i}\right\rangle z_{i} \widehat{e^{\alpha}}-\widetilde{j}^{*} \omega\left(F, h^{F}\right)_{y_{0}} .
\end{aligned}
$$

Theorem 5.5. When $t \rightarrow 0, \mathcal{L}_{t}^{(3)}$ converges to

$$
\begin{array}{r}
\mathcal{L}_{0}^{(3)}=-\left(\frac{\partial}{\partial z_{m}}-\dot{\widetilde{S}}_{s}\left(y_{0}\right)+d \bar{s} \wedge \sum_{\alpha=1}^{m-1} z_{\alpha} \mathcal{A}_{\alpha}\right)^{2}-\sum_{\alpha=1}^{m-1}\left(\frac{\partial}{\partial z_{\alpha}}+d \bar{s} \wedge z_{m} \mathcal{A}_{\alpha}\right)^{2} \\
+\frac{1}{2}\left(\dot{R}^{T \widetilde{X}} \mid \widetilde{Y}\right)_{y_{0}}+d \bar{s} \wedge \Theta(z) .
\end{array}
$$


Proof. By (5.36) and the definition of $\widetilde{e}_{\alpha}$, as in (3.62), we have for $Z \in T_{y_{0}} X$,

$$
\begin{aligned}
& \left(c\left(\widetilde{e}_{\alpha}\right)\right)_{\Phi}(z)=\left(1+\mathcal{O}\left(|z|^{2}\right)\right) c\left(e_{\alpha}\right)-\sum_{\beta=1}^{m-1}\left(A_{\alpha \beta} z_{m}+\mathcal{O}\left(|z|^{2}\right)\right) \widehat{c}\left(e_{\beta}\right) \\
& -\frac{1}{2} t^{-1 / 2}\left(\left\langle\dot{g}_{s, y_{0}}^{T X} Z, e_{\alpha}\right\rangle+\mathcal{O}\left(|z|^{2}\right)\right) d s+t^{-3 / 2} \mathcal{O}\left(|z|^{2}\right) d \bar{s}, \\
& \left(\widehat{c}\left(\widetilde{e}_{\alpha}\right)\right)_{\Phi}(z)=\left(1+\mathcal{O}\left(|z|^{2}\right)\right) \widehat{c}\left(e_{\alpha}\right)-\sum_{\beta=1}^{m-1}\left(A_{\alpha \beta} z_{m}+\mathcal{O}\left(|z|^{2}\right)\right) c\left(e_{\beta}\right) \\
& \quad-2 t^{-3 / 2}\left(z_{\alpha}+\mathcal{O}\left(|z|^{2}\right)\right) d \bar{s}-\frac{1}{2} t^{-1 / 2}\left(z_{m}\left\langle\dot{g}_{s, y_{0}}^{T X} e_{m}, e_{\alpha}\right\rangle+\mathcal{O}\left(|z|^{2}\right)\right) d s, \\
& \left(\widehat{c}\left(\widetilde{e}_{m}\right)\right)_{\Phi}(z)=\widehat{c}\left(e_{m}\right)-2 t^{-3 / 2}\left(z_{m}+\mathcal{O}\left(|z|^{2}\right)\right) d \bar{s} .
\end{aligned}
$$

From (5.47), we get for $z \in \mathbb{R}^{m}$,

$$
\begin{aligned}
& \left\{t^{1 / 2}\left(c\left(\widetilde{e}_{\alpha}\right)\right)_{\Phi}\right\}_{t}^{(3)}(z)=w\left(e^{\alpha}\right)+\mathcal{O}\left(t+t|z|^{2}\right), \\
& \left\{t^{1 / 2}\left(\widehat{c}\left(\widetilde{e}_{\alpha}\right)\right)_{\Phi}\right\}_{t}^{(3)}(z)=w\left(\widehat{e^{\alpha}}\right)-2 z_{\alpha} d \bar{s}+\mathcal{O}\left(t+t|z|^{2}\right), \\
& \left\{t^{1 / 2}\left(\widehat{c}\left(e_{m}\right)\right)_{\Phi}\right\}_{t}^{(3)}(z)=t^{1 / 2} \widehat{c}\left(e_{m}\right)-2 z_{m} d \bar{s}+\mathcal{O}\left(t|z|^{2}\right) .
\end{aligned}
$$

Let $\Gamma^{\mathcal{F}},{ }^{s p} \Gamma^{\mathcal{F}, A}$ be the connection forms of $\nabla^{\mathcal{F}},{ }^{s p} \nabla^{\mathcal{F}, A}$ on $T_{y_{0}} X$ with respect to the trivialization by parallel transport with respect to ${ }^{s p} \nabla^{\mathcal{F}, A}$ (instead of $\psi_{t}^{s p} \nabla^{\mathcal{F}, A} \psi_{t}^{-1}$ ) along the curve $\gamma_{Z}$. From (1.8), (3.23), (3.54), (5.20) and (5.35), we deduce that for $Z \in T_{y_{0}} X$,

$$
\begin{gathered}
\Gamma_{Z}^{\mathcal{F}}={ }^{s p} \Gamma_{Z}^{\mathcal{F}, A}+\frac{1}{2} \sum_{\beta=1}^{m-1}\left\langle A(\cdot) e_{m}, \widetilde{e}_{\beta}\right\rangle\left(c\left(e_{m}\right) c\left(\widetilde{e}_{\beta}\right)-\widehat{c}\left(e_{m}\right) \widehat{c}\left(\widetilde{e}_{\beta}\right)\right)-A^{\Lambda\left(T^{*} Y\right)}(\cdot) \\
+\frac{1}{4}\left\langle\dot{g}_{s}^{T X} \cdot, e_{m}\right\rangle d s \wedge c\left(e_{m}\right)+\frac{1}{4} \sum_{\beta=1}^{m-1}\left\langle\dot{g}_{s}^{T X} P^{2} T X \cdot \widetilde{e}_{\beta}\right\rangle d s \wedge \widehat{c}\left(\widetilde{e}_{\beta}\right)+\Gamma^{F, u} \cdot \quad
\end{gathered}
$$

By (3.33), (5.43), and (5.48), we obtain for $Z \in T_{y_{0}} X$ as in subsection 3.5,

$$
\begin{gathered}
\begin{array}{c}
\frac{t}{2}\left\{\left\langle A\left(\widetilde{e}_{\alpha}\right) e_{m}, \widetilde{e}_{\beta}\right\rangle\left(c\left(e_{m}\right) c\left(\widetilde{e}_{\beta}\right)-\widehat{c}\left(e_{m}\right) \widehat{c}\left(\widetilde{e}_{\beta}\right)\right)\right\}_{t}^{(3)} \\
=-z_{m} A_{\alpha \beta} d \bar{s} \wedge \widehat{e^{\beta}}+\mathcal{O}\left(\sqrt{t}+t|z|^{3}\right), \\
t\left\{A^{\Lambda\left(T^{*} Y\right)}\left(e_{m}\right)\right\}_{t}^{(3)} \\
=-\sum_{\alpha, \beta=1}^{m-1} \frac{1}{2} A_{\alpha \beta} e^{\alpha} \wedge\left(\widehat{e^{\beta}}-2 z_{\beta} d \bar{s}\right)+t \mathcal{O}\left(|z|^{3}\right)+t \mathcal{O}(1), \\
t\left\{\frac{1}{4 \sqrt{t}}\left\langle\dot{g}_{s}^{T X} e_{m}, \widetilde{e}_{\beta}\right\rangle d s \wedge \widehat{c}\left(\widetilde{e}_{\beta}\right)\right\}_{t}^{(3)} \\
=\frac{1}{4}\left\langle\dot{g}_{s}^{T X} e_{m}, e_{\beta}\right\rangle d s \wedge\left(\widehat{e^{\beta}}-2 z_{\beta} d \bar{s}\right)+\mathcal{O}\left(t+t|z|^{2}\right) .
\end{array}
\end{gathered}
$$


In analogy with (3.56)-(3.59),

$$
\begin{aligned}
{ }^{s p} \Gamma_{Z}^{\mathcal{F}, A}(U) & =\frac{1}{2}{ }^{s p} R_{y_{0}}^{\mathcal{F}, A}(Z, U)+\mathcal{O}\left(|Z|^{2}\right) U \\
& =0 \quad \text { if } Z, U \in T_{y_{0}} Y ; \\
& ={ }^{s p} R_{y_{0}}^{\mathcal{F}, A}(Z, U)+\mathcal{O}\left(|Z|^{2}\right) U \quad \text { if } Z \in T_{y_{0}} X, U \in N_{y_{0}}, U \in T_{y_{0}} Y .
\end{aligned}
$$

Observe that at $y_{0}, \nabla_{\widetilde{e}_{m}}^{T} X_{\tilde{e}_{\alpha}}={ }^{s p} \nabla_{\widetilde{e}_{m}}^{T X} \widetilde{e}_{\alpha}={ }^{s p} \nabla_{\widetilde{e}_{\alpha}}^{T X} \widetilde{e}_{m}=0$, thus

$$
\begin{aligned}
& T_{y_{0}}^{X}\left(e_{\alpha}, e_{\beta}\right)=\nabla \widetilde{e}_{\alpha}^{T Y} \widetilde{e}_{\beta}-\nabla_{\widetilde{e}_{\beta}}^{T Y} \widetilde{e}_{\alpha}-\left[\widetilde{e}_{\alpha}, \widetilde{e}_{\beta}\right]=0, \\
& T_{y_{0}}^{X}\left(e_{m}, e_{\alpha}\right)=-\left[\widetilde{e}_{m}, \widetilde{e}_{\alpha}\right]=\nabla_{\widetilde{e}_{\alpha}}^{T X} \widetilde{e}_{m}=A_{y_{0}}\left(e_{\alpha}\right) e_{m} .
\end{aligned}
$$

Now from (5.37), (5.48), and (5.52), for $Z \in T_{y_{0}} X$,

$$
\begin{aligned}
& t\left\{\psi_{t}{ }^{s p} R_{y_{0}}^{\mathcal{F}, A} \psi_{t}^{-1}\right\}_{t}^{(3)}\left(t Z, e_{\alpha}\right)=-d \bar{s} \sum_{\beta=1}^{m-1}\left\langle T_{y_{0}}^{X}\left(z_{m} e_{m}, e_{\alpha}\right), e_{\beta}\right\rangle \widehat{e^{\beta}} \\
& +z_{m} \sum_{\beta=1}^{m-1} A_{\alpha \beta} d \bar{s} \wedge e^{\beta}+\frac{1}{2} z_{m}\left\langle\dot{g}_{s, y_{0}}^{T X} e_{m}, e_{\alpha}\right\rangle d \bar{s} \wedge d s+\mathcal{O}\left(t+t|z|^{2}\right) \\
& \quad=z_{m} \sum_{\beta=1}^{m-1} A_{\alpha \beta} d \bar{s} \wedge\left(\widehat{e^{\beta}}+e^{\beta}\right)+\frac{1}{2} z_{m}\left\langle\dot{g}_{s, y_{0}}^{T X} e_{m}, e_{\alpha}\right\rangle d \bar{s} \wedge d s+\mathcal{O}\left(t+t|z|^{2}\right) .
\end{aligned}
$$

By (1.9), (5.19), (5.22), and (5.48)-(5.53), we get when $t \rightarrow 0$,

$$
\begin{aligned}
& \mathcal{L}_{t}^{(3)}(z) \rightarrow-\left(\frac{\partial}{\partial z_{m}}-\dot{\widetilde{S}}_{s}\left(y_{0}\right)+d \bar{s} \wedge \sum_{\alpha=1}^{m-1} z_{\alpha} \mathcal{A}_{\alpha}\right)^{2}-\sum_{\alpha=1}^{m-1}\left(\frac{\partial}{\partial z_{\alpha}}+d \bar{s} \wedge z_{m} \mathcal{A}_{\alpha}\right)^{2} \\
&+ \frac{1}{4} \sum_{\alpha, \beta=1}^{m-1}\left\langle e_{\alpha},\left(\widetilde{j}^{*} R^{T \widetilde{X}}\right)_{y_{0}} e_{\beta}\right\rangle\left(\widehat{e^{\alpha}}-2 z_{\alpha} d \bar{s}\right) \wedge\left(\widehat{e^{\beta}}-2 z_{\beta} d \bar{s}\right) \\
&+\frac{1}{2} \sum_{\gamma=1}^{m-1}\left\langle e_{\gamma},\left(\widetilde{j}^{*} R^{T \widetilde{X}}\right)_{y_{0}} e_{m}\right\rangle \widehat{e^{\gamma}} \wedge\left(-2 z_{m} d \bar{s}\right) \\
&-d \bar{s} \wedge \widetilde{j}^{*} \omega\left(F, h^{F}\right)_{y_{0}}=\mathcal{L}_{0}^{(3)}(z) .
\end{aligned}
$$

The proof of (5.46) is complete.

Let $e^{-\mathcal{L}_{t}^{(3)}}(z, w)\left(z, w \in \mathbb{R}^{m}\right)$ and $e^{-\mathcal{L}_{t, a}^{(3)}}(z, w)\left(z, w \in \mathbb{R}_{+}^{m}\right)$ be the smooth kernels of $e^{-\mathcal{L}_{t}^{(3)}}$ and $e^{-\mathcal{L}_{t, a}^{(3)}}$, respectively, calculated with respect to $d v_{T_{y_{0} X} X}(w)$. These kernels have the same structure as their counterparts in subsection 3.6, and the technique used there also applies to $\mathcal{L}_{t}^{(3)}$. Thus we get the following technical result: 
Theorem 5.6. There exist $r \in \mathbb{N}, C_{0}>0$ such that for any $l \in \mathbb{N}$, there exists $C>0$ such that for $t \in(0,1], z, w>\mathbb{R}_{+}^{m}$,

$$
\left|z_{m}^{l}\left(e^{-\mathcal{L}_{t, a}^{(3)}}-e^{-\mathcal{L}_{t}^{(3)}}\right)(z, w)\right| \leq C(1+|z|+|w|)^{r} e^{-C_{0}|z-w|^{2}} .
$$

Furthermore, for $z, w \in \mathbb{R}_{+}^{m}$,

$$
\lim _{t \rightarrow 0} e^{-\mathcal{L}_{t, a}^{(3)}}(z, w)=e^{-\mathcal{L}_{0, a}^{(3)}}(z, w), \quad \lim _{t \rightarrow 0} e^{-\mathcal{L}_{t}^{(3)}}(z, w)=e^{-\mathcal{L}_{0}^{(3)}}(z, w) .
$$

5.4 Limit of the boundary contribution $\mathfrak{J}_{t}(y)$ in $(5.34)$ when $\boldsymbol{t} \rightarrow \mathbf{0}$. Recall the fundamental solutions $F_{\alpha}, F_{D i r}, K$ are defined in (3.121), (3.122), and (3.127).

\section{Theorem 5.7.}

$$
\lim _{t \rightarrow 0} \mathcal{J}_{t}\left(y_{0}\right) d v_{Y}\left(y_{0}\right)=\left[e_{b}\left(Y \times \mathbb{R}, \nabla^{T \tilde{X}}\right)\right]^{d s} \widetilde{j}^{*} \theta\left(F, h^{F}\right)+\operatorname{rk}(F)\left[\mu\left(\nabla^{T} \tilde{X}\right)\right]^{d s} \text {. }
$$

Proof. By the equations analogous to (3.48)-(3.50), (3.131), (5.34) and Theorem 5.6, we get

$$
\begin{aligned}
& \lim _{t \rightarrow 0} \mathcal{J}_{t}\left(y_{0}\right)=2^{m-1}(-1)^{\frac{m(m-1)}{2}} \\
& \quad \int_{0}^{\infty} \operatorname{Tr}_{s}\left[\left[\left(e^{-\mathcal{L}_{0, a}^{(3)}}-e^{-\mathcal{L}_{0}^{(3)}}\right)\left(\left(0, z_{m}\right),\left(0, z_{m}\right)\right)\right]^{\max }\right]^{d \bar{s} d s} d z_{m} .
\end{aligned}
$$

By (5.46), $\quad\left[e^{-\mathcal{L}_{0}^{(3)}}(z, z)\right]^{\max } \quad$ acts $\quad$ on $\Lambda\left(N^{*}\right)$ as $c \operatorname{Id}_{\Lambda\left(N^{*}\right)}$, thus $\operatorname{Tr}_{s}\left[e^{-\mathcal{L}_{0}^{(3)}}(z, z)\right]^{\max }=0$. Now we need to calculate the kernel of the heat operator $e^{-\mathcal{L}_{0, a}^{(3)}}$. Let $\bar{F}, \bar{H}$ be the sections of

$$
\left.\left(\Lambda(\mathbb{C}(d s)) \widehat{\otimes} \Lambda\left(T^{*} Y\right) \widehat{\otimes} \widehat{\Lambda\left(T^{*} Y\right.}\right) \widehat{\otimes} \operatorname{End}\left(\Lambda\left(N^{*}\right)\right) \otimes \operatorname{End}(F)\right)_{y_{0}}
$$
on $\mathbb{R}_{+}^{m} \times \mathbb{R}_{+}^{m}$ such that

$$
\begin{array}{r}
\left.e^{-t \mathcal{L}_{0, a}^{(3)}(z, w)=\exp (}\left(z_{m}-w_{m}\right) \dot{\widetilde{S}}_{s}\left(y_{0}\right)-d \bar{s} \wedge \sum_{\alpha}\left(z_{m} z_{\alpha}-w_{m} w_{\alpha}\right) \mathcal{A}_{\alpha}\right) \\
\times \exp \left(-\frac{t}{2}\left(\dot{R}^{T \widetilde{X}} \mid \widetilde{Y}\right)_{y_{0}}\right)[\bar{F}+d \bar{s} \wedge \bar{H}] .
\end{array}
$$

Then from (5.44), (5.46), $\bar{F}+d \bar{s} \wedge \bar{H}$ is the fundamental solution of the following equation:

$$
\left\{\begin{array}{l}
\left.i\left(e_{m}\right) \sigma\right|_{z_{m}=0}=0 \\
\left.w\left(e^{m}\right)\left[\nabla_{e_{m}}+\dot{\widetilde{S}}_{s}\left(y_{0}\right)-d \bar{s} \wedge \sum_{\alpha=1}^{m-1} z_{\alpha} \mathcal{A}_{\alpha}\right] \sigma\right|_{z_{m}=0}=0, \\
\left(\frac{\partial}{\partial t}+\Delta^{T_{y_{0}} X}+d \bar{s} \wedge \Theta(z)\right) \sigma=0 .
\end{array}\right.
$$

By (5.60), $\bar{F}$ is the fundamental solution of the equation (3.129), thus $\bar{F}=Q$ defined by (3.132) and (3.135). As in (3.132), set

$$
\bar{H}=\bar{H}_{1} w\left(e^{m}\right)+\bar{H}_{2} i\left(e_{m}\right)+\bar{H}_{3} w\left(e^{m}\right) i\left(e_{m}\right)+\bar{H}_{4} i\left(e_{m}\right) w\left(e^{m}\right),
$$


where $\left.\bar{H}_{i} \in\left(\Lambda(\mathbb{C}(d s)) \widehat{\otimes} \Lambda\left(T^{*} Y\right) \widehat{\otimes} \widehat{\Lambda\left(T^{*} Y\right.}\right) \otimes \operatorname{End}(F)\right)_{y_{0}}(1 \leq i \leq 4)$. By (5.60) and (5.61) and the uniqueness of the solution, we get $\bar{H}_{1}=\bar{H}_{2}=0$. Turning to the coefficient of $d \bar{s}$ in (5.60), for $\psi$ any smooth section of $\left.\left(\Lambda(\mathbb{C}(d s)) \widehat{\otimes} \Lambda\left(T^{*} Y\right) \widehat{\otimes} \widehat{\Lambda\left(T^{*} Y\right.}\right) \otimes F\right)_{y_{0}}$ with compact support on $\mathbb{R}_{+}^{m}$, for $i=3,4$, we find from $(5.60),(5.61)$,

$$
\left\{\begin{array}{l}
\left.\bar{H}_{i} \psi\right|_{t=0}=0, \\
\left.\bar{H}_{3} \psi\right|_{z_{m}=0}=0,\left[\nabla_{e_{m}}+\dot{\widetilde{S}}_{s}\left(y_{0}\right)\right] \bar{H}_{4} \psi-\left.\sum_{\alpha=1}^{m-1} \mathcal{A}_{\alpha} z_{\alpha} Q_{4} \psi\right|_{z_{m}=0}=0, \\
\left(\frac{\partial}{\partial t}+\Delta^{T_{y_{0}} X}\right) \bar{H}_{i} \psi+\Theta(z) Q_{i} \psi=0 .
\end{array}\right.
$$

Thus by (1.17), and (5.58), we get

$$
\begin{aligned}
\lim _{t \rightarrow 0} \mathcal{J}_{t}\left(y_{0}\right)=2^{m-1}( & -1)^{\frac{m(m-1)}{2}} \int_{0}^{\infty} d z_{m}\left[\left[\exp \left(-\frac{1}{2}\left(\dot{R}^{T \tilde{X}} \mid \widetilde{Y}\right)_{y_{0}}\right)\right.\right. \\
\times & \left.\left.\operatorname{Tr}_{F}\left[\left(\bar{H}_{4}-\bar{H}_{3}\right)\left(1,\left(0, z_{m}\right),\left(0, z_{m}\right)\right)\right]\right]^{\max }\right]^{d s} .
\end{aligned}
$$

By solving the inhomogeneous problem (5.62) for $i=3$, we get

$$
\bar{H}_{3}(t, z, w)=-\int_{0}^{t} d \tau \int_{\mathbb{R}_{+}^{m}} Q_{3}(t-\tau, z, x) \Theta(x) Q_{3}(\tau, x, w) d x .
$$

From Proposition 3.21,

$$
\lim _{x \searrow 0} \int_{0}^{t} \frac{\partial}{\partial x} F_{\dot{\tilde{S}}}(t-\tau, x, 0) \varphi(\tau) d \tau=-\varphi(t)-\dot{\widetilde{S}} \int_{0}^{t} F_{\dot{\tilde{S}}}(t-\tau, 0,0) \varphi(\tau) d \tau .
$$

Similarly, by solving the inhomogeneous problem (5.62) for $i=4$, using (5.65), we get

$$
\begin{aligned}
& \bar{H}_{4}(t, z, w)=-\int_{0}^{t} d \tau \int_{\mathbb{R}_{+}^{m}} Q_{4}(t-\tau, z, x) \Theta(x) Q_{4}(\tau, x, w) d x \\
& -\int_{0}^{t} d \tau \int_{\mathbb{R}^{m-1}} Q_{4}\left(t-\tau, z,\left(x^{\prime}, 0\right)\right) \sum_{\alpha=1}^{m-1} \mathcal{A}_{\alpha} x_{\alpha} \wedge Q_{4}\left(\tau,\left(x^{\prime}, 0\right), w\right) d x^{\prime} .
\end{aligned}
$$

By (3.127), (3.135), (5.64) and (5.66),

$$
\begin{aligned}
& \bar{H}_{3}\left(t,\left(0, z_{m}\right),\left(0, z_{m}\right)\right) \\
& =-K(t, 0,0) \int_{0}^{t} d \tau \int_{0}^{\infty} F_{D i r}\left(t-\tau, z_{m}, x_{m}\right) \Theta\left(0, x_{m}\right) F_{D i r}\left(\tau, x_{m}, z_{m}\right) d x_{m}, \\
& \bar{H}_{4}\left(t,\left(0, z_{m}\right),\left(0, z_{m}\right)\right) \\
& =-K(t, 0,0) \int_{0}^{t} d \tau \int_{0}^{\infty} F_{\tilde{S}_{s}}\left(t-\tau, z_{m}, x_{m}\right) \Theta\left(0, x_{m}\right) F_{\tilde{S}_{s}}\left(\tau, x_{m}, z_{m}\right) d x_{m} .
\end{aligned}
$$


By the semi-group property of heat operators, we have for $\mathbf{F}=F_{\text {Dir }}$ or $F_{\dot{\tilde{S}}}$,

$$
\int_{0}^{\infty} \mathbf{F}(t-\tau, y, x) \mathbf{F}\left(\tau, x, y^{\prime}\right) d x=\mathbf{F}\left(t, y, y^{\prime}\right) .
$$

As $F_{D i r}, F_{\dot{\tilde{S}}}$ act on $F_{y_{0}}$ as multiplication of the identity, by (3.125), (3.126), (5.45), (5.67), and (5.68), we get

$$
\begin{aligned}
\int_{0}^{\infty} & \left(\bar{H}_{4}-\bar{H}_{3}\right)\left(t,\left(0, z_{m}\right),\left(0, z_{m}\right)\right) d z_{m} \\
= & -K(t, 0,0) \int_{0}^{\infty} d x_{m} \int_{0}^{t} d \tau \Theta\left(0, x_{m}\right)\left(F_{\dot{\tilde{S}}_{s}}-F_{D i r}\right)\left(t, x_{m}, x_{m}\right) \\
= & (4 \pi t)^{-\frac{m-1}{2}}\left\{\widetilde{j}^{*} \omega\left(F, h^{F}\right)_{y_{0}} \sum_{k=0}^{\infty} \frac{\dot{\widetilde{S}}_{s}^{k} t^{(k+2) / 2}}{2 \Gamma\left(\frac{k}{2}+1\right)}\right. \\
& \left.-\sum_{\alpha=1}^{m-1}\left\langle e_{\alpha},\left(\widetilde{j}^{*} R^{T \widetilde{X}}\right)_{y_{0}} e_{m}\right\rangle \widehat{e^{\alpha}} \wedge \sum_{k=1}^{\infty} \frac{\dot{\vec{S}}_{s}^{k-1} t^{(k+2) / 2}}{4 \Gamma\left(\frac{k}{2}+1\right)}\right\} .
\end{aligned}
$$

By (1.14) and (1.17),

$$
\begin{gathered}
-\int^{B_{Y}} \exp \left(-\frac{1}{2} \dot{R}^{T \widetilde{X}} \mid \widetilde{Y}\right) \sum_{\alpha=1}^{m-1}\left\langle e_{\alpha}, \widetilde{j}^{*} R^{T \widetilde{X}} e_{m}\right\rangle \widehat{e^{\alpha}} \wedge \sum_{k=1}^{\infty} \frac{\dot{\widetilde{S}}_{s}^{k-1}}{4 \Gamma\left(\frac{k}{2}+1\right)} \\
=\sqrt{\pi} \int^{B_{X}} \exp \left(-\frac{1}{2} \widetilde{j}^{*} \dot{R}^{T \widetilde{X}}\right) \sum_{k=1}^{\infty} \frac{\dot{\vec{S}}_{s}^{k-1}}{2 \Gamma\left(\frac{k}{2}+1\right)}=\mu\left(\nabla^{T \widetilde{X}}\right) .
\end{gathered}
$$

Thus from (1.17), (5.63), (5.69), and (5.70) we get (5.57).

5.5 Proof of Theorem 0.1. We now summarize our work and show how the various arguments given above combine to furnish the proof of Theorem 0.1. Recall that we are given a manifold $X$ with boundary $Y$, a flat vector bundle $F$ over $X$, and smooth families of metrics $\left(g_{s}^{T X}, h_{s}^{F}\right)_{s \in[0,1]}$ on $T X$ and $F$, respectively, which satisfy no additional assumptions.

Our starting point is the variational formula given in Theorem 4.5 which links the variation of the induced Ray-Singer metrics to a "weighted" heat trace expansion. For convenience of notation we will now write l.i.m.t $t \rightarrow 0$ to denote the constant term in an asymptotic expansion with respect to the parameter $t$. Then Theorem 4.5 reads

$$
\begin{aligned}
\frac{\partial}{\partial s} \log \left(\left(\|\|_{\operatorname{det} H \bullet(X, F), s}^{\mathrm{RS}}\right)^{2}\right) & =1 . i \cdot \mathrm{m}_{\cdot t \rightarrow 0} \operatorname{Tr}_{s}\left[\left(*_{s}^{-1} \frac{\partial *_{s}}{\partial s}+\left(h_{s}^{F}\right)^{-1} \frac{\partial h_{s}^{F}}{\partial s}\right) e^{-t D_{s, a}^{2}}\right] \\
& =: M_{0, s}^{g}+M_{0, s}^{h} \\
& =: M_{0, s} .
\end{aligned}
$$


We compute the second contribution first; in fact, using the notation introduced in $(0.2),(0.3)$, we find from $(4.22 \mathrm{~b})$

$$
M_{0, s}^{h}=(-1)^{m} \int_{(X, Y)} \operatorname{Tr}\left[\left(h_{s}^{F}\right)^{-1} \frac{\partial}{\partial s} h_{s}^{F}\right] E\left(T X, \nabla_{s}^{T X}\right) .
$$

To deal with the first term, we relate the "weighted" heat trace expansion to a "true" heat trace, namely for the operator $\mathcal{L}_{t}^{(0)}$ introduced in $(5.3 \mathrm{~b})$; this involves the introduction of a new Grassmann variable $d \bar{s}$. Using the notation introduced at the beginning of section 5 , and denoting by $\mathcal{L}_{t, a}^{(0)}$ the operator $\mathcal{L}_{t}^{(0)}$ with the appropriate boundary condition, we find in (5.9) that

$$
M_{0, s}^{g}=\text { l.i.m.t } \rightarrow 0 \operatorname{Tr}_{s}\left[e^{-\mathcal{L}_{t, a}^{(0)}}\right]^{d \bar{s} d s} .
$$

Next we may replace $\mathcal{L}_{t, a}^{(0)}$ by $\mathcal{L}_{t}^{(0)}$ in the part of $X$ with boundary distance $\geq t^{1 / 4}$, in view of the fundamental estimate (5.26) (expressing the fact that the associated wave equation has finite propagation speed). Then, by standard techniques in local index theory, we calculate in (5.33) the "inner" contribution, $M_{0, s}^{g, i}$, to $M_{0, s}^{g}$ as

$$
M_{0, s}^{g, i}=\int_{X}\left[e\left(T \widetilde{X}, \nabla^{T \tilde{X}}\right)\right]^{d s} \wedge \theta\left(F, h_{s}^{F}\right),
$$

where $\nabla^{T} \tilde{X}$ is the connection on $T \widetilde{X}$ canonically associated to the family $\left(g_{s}^{T X}\right)$. Finally, we use a somewhat strange rescaling and solve the resulting model boundary value problem to get the boundary contribution, $M_{0, s}^{g, b}$, which is calculated in Theorem 5.7 (which uses the notation (5.34)) as

$$
M_{0, s}^{g, b}=\int_{Y}\left\{\left[e_{b}\left(Y \times \mathbb{R}, \nabla^{T \widetilde{X}}\right)\right]^{d s} \wedge j^{*} \theta\left(F, h_{s}^{F}\right)+\operatorname{rk}(F)\left[\mu\left(\nabla^{T \widetilde{X}}\right)\right]^{d s}\right\} \text {. }
$$

Writing now

$$
\hat{E}\left(T \tilde{X}, \nabla^{T \tilde{X}}\right):=\left(e\left(T \tilde{X}, \nabla^{T \widetilde{X}}\right),-e_{b}\left(Y \times \mathbb{R}, \nabla^{T \widetilde{X}}\right)\right),
$$

these identities combine to

$$
\begin{aligned}
& M_{0, s}=(-1)^{m} \int_{(X, Y)} \operatorname{Tr}\left[\left(h_{s}^{F}\right)^{-1} \frac{\partial}{\partial s} h_{s}^{F}\right] E\left(T X, \nabla_{s}^{T X}\right) \\
& \quad+\int_{(X, Y)}\left[\hat{E}\left(T \widetilde{X}, \nabla^{T \widetilde{X}}\right)\right]^{d s} \wedge \theta\left(F, h_{s}^{F}\right)+\operatorname{rk}(F) \int_{Y}\left[\mu\left(\nabla^{T \widetilde{X}}\right)\right]^{d s} .
\end{aligned}
$$

It remains to evaluate the $s$-integral of $M_{0, s}$ over $[0,1]$. To do so, we first assume that the path in the space of metrics connecting $\left(g_{0}^{T X}, h_{0}^{F}\right)$ and $\left(g_{1}^{T X}, h_{1}^{F}\right)$ is chosen in such a way that $g_{s}^{T X}=g_{0}^{T X}$ in a neighborhood of $[0,1 / 2]$, and $h_{s}^{F}=h_{1}^{F}$ in a neighborhood of $[1 / 2,1]$. Then we can carry out the integration, observing that

$$
\left[\hat{E}\left(T \widetilde{X}, \nabla^{T \widetilde{X}}\right)\right]^{d s}=0, \quad s \in[0,1 / 2] .
$$


Employing the notation introduced in Definition 1.8 and using (1.49), we arrive at the proof of Theorem 0.1.

Now we may indeed choose any path connecting $\left(g_{0}^{T X}, h_{0}^{F}\right)$ and $\left(g_{1}^{T X}, h_{1}^{F}\right)$ in the space of metrics if we can show that the right-hand side in Theorem 0.1 does not depend on this choice. This is obvious for the third term, by (1.49). For the sum of the first and the second term we argue as follows.

Denote by $\mathcal{M}_{F}, \mathcal{M}$ the spaces of (Hermitian resp. Euclidean) metrics on $F, T X$. Then we have a fibration $\tilde{\pi}: \mathcal{W}=X \times \mathcal{M} \times \mathcal{M}_{F} \rightarrow \mathcal{M} \times \mathcal{M}_{F}$. Let $p: \mathcal{W} \rightarrow X$ be the obvious projection, and put $T^{H} \mathcal{W}:=\widetilde{\pi}^{*} T \mathcal{M} \oplus \widetilde{\pi}^{*} T \mathcal{M}_{F}$. Then $\widetilde{F}=p^{*} F$ is a flat vector bundle on $\mathcal{W}$ with the tautological metric $h^{\widetilde{F}}$. Using (4.5), we find

$$
\theta\left(\widetilde{F}, h^{\widetilde{F}}\right)=p^{*} \theta\left(F, h^{F}\right)+\operatorname{Tr}\left[\left(h^{F}\right)^{-1} d^{\mathcal{M}_{F}} h^{F}\right] .
$$

Thus we may rewrite the first two terms in (5.76), using (1.51), and (5.77), in the form

$$
\begin{aligned}
(-1)^{m} \int_{(X, Y)} \operatorname{Tr}\left[\left(h_{s}^{F}\right)^{-1} \frac{\partial}{\partial s} h_{s}^{F}\right] E\left(T X, \nabla_{s}^{T X}\right) & \\
& +\int_{(X, Y)}\left[\hat{E}\left(T \widetilde{X}, \nabla^{T \widetilde{X}}\right)\right]^{d s} \wedge \theta\left(F, h_{s}^{F}\right) \\
& =\left[\int_{(X, Y)} E\left(T \widetilde{X}, \nabla^{T \widetilde{X}}\right) \theta\left(\widetilde{F}, h^{\widetilde{F}}\right)\right]^{d s} .
\end{aligned}
$$

In view of Remark 1.10, the right-hand side of (5.78) is the fiber integral of a closed $\mathrm{m}+1$-form on $(\widetilde{X}, \widetilde{Y}) \times \mathcal{M} \times \mathcal{M}_{F}$ over the fiber $(\widetilde{X}, \widetilde{Y})$ such that the path integral of the resulting one-form on $\mathcal{M} \times \mathcal{M}_{F}$ depends only on the endpoints. This completes the proof of Theorem 0.1.

5.6 Proof of Theorem 4.2. We choose the family of metrics such that $g_{s}^{T X}=s g^{T X}, h_{s}^{F}=h^{F}$ near 1 . Recall that $p_{X}: X \times \mathbb{R} \rightarrow X$ is the natural projection. Then by (1.12), (1.44) and (4.20), for $y \in Y$, near $s=1$,

$$
\begin{gathered}
R^{T \widetilde{X}}=p_{X}^{*} R^{T X}, \quad \dot{\widetilde{S}}(y, s)=s \dot{S}(y), \\
\left.*_{s}^{-1} \frac{\partial *_{s}}{\partial s}\right|_{s=1}=-\frac{1}{2} \sum_{i=1}^{m} c\left(e_{i}\right) \widehat{c}\left(e_{i}\right)=-N+\frac{m}{2} .
\end{gathered}
$$

By (3.9), (4.6), (4.22a), and (5.79), we know

$$
\begin{aligned}
\operatorname{Tr}_{s}\left[N e^{-t^{2} D_{a}^{2}}\right] & =-\operatorname{Tr}_{s}\left[\left.*_{s}^{-1} \frac{\partial *_{s}}{\partial s}\right|_{s=1} e^{-t^{2} D_{a}^{2}}\right]+\frac{m}{2} \operatorname{Tr}_{s}\left[e^{-t^{2} D_{a}^{2}}\right] \\
& =-\frac{1}{t} M_{-1,1}-M_{0,1}+\frac{m}{2} \chi(X, F)+\mathcal{O}(t) .
\end{aligned}
$$

By (4.22a), $-M_{-1,1}$ is given by $a_{-1}$ in (4.2). By (1.17), (5.76) and (5.79), we get $M_{0,1}=0$. We complete the proof of Theorem 4.2. 


\section{References}

[ABP] M.F. AtiYah, R. Bott, V.K. Patodi, On the heat equation and the index theorem, Invent. Math. 19 (1973), 279-330.

[APS] M.F. Atiyah, V.K. Patodi, I.M. Singer, Spectral asymmetry and Riemannian geometry. I, Math. Proc. Cambridge Philos. Soc. 77 (1975), 43-69.

[BGV] N. Berline, E. Getzler, M. Vergne, Heat Kernels and Dirac Operators, Springer Grundl. Math. Wiss. 298 (1992).

[Bi1] J.-M. Bismut, The index theorem for families of Dirac operators: two heat equation proofs, Invent. Math. 83 (1986), 91-151.

[Bi2] J.-M. Bismut, Equivariant immersions and Quillen metrics, J. Diff. Geom. 41 (1995), 53-159.

[Bi3] J.-M. Bismut, Families of Immersions, and Higher Analytic Torsion, Astérisque 244 (1997).

[BiGS1] J.-M. Bismut, H. Gillet, C. Soulé, Analytic torsion and holomorphic determinant bundles II, Comm. Math. Phys. 115 (1988), 79-126.

[BiGS2] J.-M. Bismut, H. Gillet, C. Soulé, Analytic torsion and holomorphic determinant bundles III, Comm. Math. Phys. 115 (1988), 301-351.

[BiG] J.-M. Bismut, S. Goette, Families Torsion and Morse Functions, Astérisque 275 (2001).

[BiL] J.-M. Bismut, G. Lebeau, Complex immersions and Quillen metrics, Publ. Math. IHES 74 (1991), 1-297.

[BiLo] J.-M. Bismut, J. LotT, Flat vector bundles, direct images and higher real analytic torsion, JAMS 8 (1995), 291-363.

[BiZ1] J.-M. Bismut, W. Zhang, An Extension of a Theorem by Cheeger and Müller, Astérisque 205 (1992).

[BiZ2] J.-M. Bismut, W. Zhang, Milnor and Ray-Singer metrics on the equivariant determinant of a flat vector bundle. GAFA, Geom. funct. anal. 4 (1994), 136-212.

[BoT] R. Bотт, L. Tu, Differential Forms in Algebraic Topology, Springer GTM $82(1982)$.

[BrG] T. Branson, P. Gilkey, The asymptotics of the Laplacian on a manifold with boundary, Comm. Partial Differential Equations 15 (1990), 245-272.

[Brü] J. BRÜNING, The local index theorem without smoothness, in "Partial Differential Equations and Mathematical Physics (Copenhagen, 1995; Lund, 1995)", Progr. Nonlinear Differential Equations Appl., 21, Birkhäuser Boston, Boston, MA (1996), 58-91.

[BrüL] J. Brüning, M. Lesch, Hilbert complexes. J. Funct. Anal. 108 (1992), $88-132$.

[BrüM1] J. BRÜNING, X. MA, An anomaly formula for Ray-Singer metrics on manifolds with boundary, C.R. Acad. Sci. Paris, Ser. I, 335 (2002), 603608. 
[BrüM2] J. BRÜNING, X. MA, On the gluing formula for the analytic torsion, to appear.

[C] M. DO CARMo, Riemannian Geometry, Birkhäuser Boston, Inc., Boston, MA, 1992.

[CaJ] H.S. Carslaw, J.C. Jeager, Conduction of Heat in Solids, Oxford, 1959.

[Ch] J. Cheeger, Analytic torsion and the heat equation, Ann. of Math. 109 (1979), 259-322.

[Che1] S.S. Chern, A simple intrinsic proof of the Gauss-Bonnet formula for closed Riemannian manifolds, Ann. of Math. 45 (1944), 747-752.

[Che2] S.S. Chern, On the curvatura integra in a Riemannian manifold, Ann. of Math. 46 (1945), 674-684.

[CheS] S.S. Chern, J. Simons, Characteristic forms and geometric invariants, Ann. Math. (2) 99 (1974), 48-69.

[DF] X. DAI, H. FAnG, Analytic torsion and R-torsion for manifolds with boundary, Asian J. Math 4 (2000), 695-714.

[F] A. Friedman, Partial Differential Equations of Parabolic Type, PrenticeHall, Inc., Englewood Cliffs, N.J. 1964.

[G] P.B. Gilkey, Invariance Theory, the Heat Equation, and the AtiyahSinger Index Theorem, Publish or Perish, Wilmington, 1984.

[Go] S. Goette, Morse theory and higher torsion invariants I, math/0111222.

[Gr] P. Greiner, An asymptotic expansion for the heat equation, Arch. Rational Mech. Anal. 41 (1971), 163-218.

[H] A. Hassell, Analytic surgery and analytic torsion, Comm. Anal. Geom. 6 (1998), 255-289.

[LR] J. Lott, M. Rothenberg, Analytic torsion for group actions, J. Diff. Geom. 34 (1991), 431-481.

[Lü] W. LüCK, Analytic and topological torsion for manifolds with boundary and symmetry, J. Diff. Geom. 37 (1993), 263-322.

[LüS] W. LüCK, T. SchICK, $L^{2}$-torsion of hyperbolic manifolds of finite volume, GAFA, Geom. funct. anal. 9 (1999), 518-567.

[M] X. MA, Functoriality of real analytic torsion forms, Israel J. Math, 131 (2002), 1-50.

[MQ] V. Mathai, D. Quillen, Superconnections, Thom classes, and equivariant differential forms, Topology 25 (1986), 85-110.

[Mi] J. Milnor, Whitehead torsion. Bull. Amer. Math. Soc. 72 (1966), 358426.

[Mü1] W. MüLLER, Analytic torsion and R-torsion of Riemannian manifolds, Adv. in Math. 28 (1978), 233-305.

[Mü2] W. MüLLER, Analytic torsion and R-torsion for unimodular representations, JAMS 6 (1993), 721-753.

[P] V.K. PATOdI, Curvature and the eigenforms of the Laplace operator, J. Diff. Geom. 5 (1971), 233-249. 
[RS] D.B. RAY, I.M. Singer, R-torsion and the Laplacian on Riemannian manifolds, Adv. in Math. 7 (1971), 145-210.

[S] R.T. SEELEy, Analytic extension of the trace associated with elliptic boundary problems, Amer. J. Math. 91 (1969), 963-983.

[T] M. TAYLOR, Partial Differential Equations I. Basic Theory, Applied Mathematical Sciences 115, Springer 1996.

[V] S.M. VishiK, Generalized Ray-Singer conjecture I: a manifold with a smooth boundary, Comm. Math. Phys. 167 (1995), 1-102.

[Z] W. Zhang, Lectures on Chern-Weil Theory and Witten Deformations, Nankai Tracts in Mathematics, 4. World Scientific, 2001.

Jochen BRÜNIng, Institut für Mathematik der Humboldt-Universität zu Berlin, Rudower Chaussee 25, 12489 Berlin, Germany

bruening@mathematik.hu-berlin.de

XiaOnan MA, UMR 7640 du CNRS, Centre de Mathématiques, École Polytechnique, 91128 Palaiseau Cedex, France

ma@math.polytechnique.fr

Received: January 2004

Revision: February 2005

Accepted: September 2005 